\title{
Local controllability to trajectories for non-homogeneous incompressible Navier-Stokes equations
}

\author{
Mehdi Badra $^{a}$, Sylvain Ervedoza ${ }^{\mathrm{b}, *}$, Sergio Guerrero ${ }^{\mathrm{c}}$ \\ ${ }^{a}$ Laboratoire LMAP, UMR CNRS 5142, Université de Pau et des Pays de l'Adour, F-64013 Pau Cedex, France \\ b Institut de Mathématiques de Toulouse; UMR5219; Université de Toulouse; CNRS; UPS IMT, F-31062 Toulouse Cedex 9, France \\ ${ }^{\mathrm{c}}$ Université Pierre et Marie Curie, UMR 7598, Laboratoire Jacques-Louis Lions, F-75005 Paris, France
}

Received 14 March 2014; received in revised form 21 October 2014; accepted 11 November 2014

Available online 3 December 2014

\begin{abstract}
The goal of this article is to show a local exact controllability to smooth $\left(C^{2}\right)$ trajectories for the density dependent incompressible Navier-Stokes equations. Our controllability result requires some geometric condition on the flow of the target trajectory, which is remanent from the transport equation satisfied by the density. The proof of this result uses a fixed point argument in suitable spaces adapted to a Carleman weight function that follows the flow of the target trajectory. Our result requires the proof of new Carleman estimates for heat and Stokes equations.

(c) 2014 L'Association Publications de l'Institut Henri Poincaré. Published by Elsevier B.V. All rights reserved.
\end{abstract}

Keywords: Non-homogeneous Navier-Stokes equations; Local exact controllability to trajectories; Carleman estimates

\section{Introduction}

The goal of this article is to discuss the local exact controllability property for the non-homogeneous Navier-Stokes equations.

Setting and main results. Let $\Omega$ be a smooth bounded domain of $\mathbb{R}^{d}, d \in\{2,3\}, T>0$ and denote $(0, T) \times \Omega$ by $\Omega_{T}$. Let us consider a trajectory $(\bar{\sigma}, \overline{\mathbf{y}})$ of the non-homogeneous Navier-Stokes equations:

$$
\begin{cases}\partial_{t} \bar{\sigma}+\operatorname{div}(\bar{\sigma} \overline{\mathbf{y}})=\bar{f}_{\sigma} & \text { in } \Omega_{T}, \\ \bar{\sigma} \partial_{t} \overline{\mathbf{y}}+\bar{\sigma}(\overline{\mathbf{y}} \cdot \nabla) \overline{\mathbf{y}}-v \Delta \overline{\mathbf{y}}+\nabla \bar{q}=\overline{\mathbf{f}}_{\mathbf{y}} & \text { in } \Omega_{T}, \\ \operatorname{div} \overline{\mathbf{y}}=0 & \text { in } \Omega_{T}, \\ (\bar{\sigma}(0), \overline{\mathbf{y}}(0))=\left(\bar{\sigma}_{0}, \overline{\mathbf{y}}_{0}\right) & \text { in } \Omega\end{cases}
$$

\footnotetext{
This work is partially supported by the Agence Nationale de la Recherche (ANR, France), Project CISIFS number NT09-437023.

* Corresponding author at: Institut de Mathématiques de Toulouse, Université Paul Sabatier, 118 route de Narbonne, 31062 Toulouse Cedex 9 , France. Tel.: +33 561557654 .

E-mail addresses: mehdi.badra@univ-pau.fr (M. Badra), sylvain.ervedoza@math.univ-toulouse.fr (S. Ervedoza), guerrero@ ann.jussieu.fr (S. Guerrero).
} 
Here, $v>0$ is the viscosity parameter and the source terms $\left(\bar{f}_{\sigma}, \overline{\mathbf{f}}_{\mathbf{y}}\right)$ are assumed to be known.

We will focus on the local exact controllability problem around the trajectory $(\bar{\sigma}, \overline{\mathbf{y}})$ with a control exerted on the boundary $(0, T) \times \partial \Omega$ : Given $\left(\rho_{0}, \mathbf{u}_{0}\right)$ small, find control functions $\left(h_{\sigma}, \mathbf{h}_{\mathbf{y}}\right)$ on $(0, T) \times \partial \Omega$ such that the solution $(\sigma, \mathbf{y})$ of

$$
\begin{cases}\partial_{t} \sigma+\operatorname{div}(\sigma \mathbf{y})=\bar{f}_{\sigma} & \text { in } \Omega_{T}, \\ \sigma \partial_{t} \mathbf{y}+\sigma(\mathbf{y} \cdot \nabla) \mathbf{y}-v \Delta \mathbf{y}+\nabla q=\overline{\mathbf{f}}_{\mathbf{y}} & \text { in } \Omega_{T}, \\ \operatorname{div} \mathbf{y}=0 & \text { in } \Omega_{T}, \\ (\sigma(0), \mathbf{y}(0))=\left(\bar{\sigma}_{0}+\rho_{0}, \overline{\mathbf{y}}_{0}+\mathbf{u}_{0}\right), & \text { in } \Omega,\end{cases}
$$

with the boundary conditions:

$$
\begin{aligned}
& \sigma=\bar{\sigma}+h_{\sigma} \quad \text { for }(t, x) \in(0, T) \times \partial \Omega, \text { with } \mathbf{y}(t, x) \cdot \mathbf{n}(x)<0, \\
& \mathbf{y}=\overline{\mathbf{y}}+\mathbf{h}_{\mathbf{y}} \quad \text { on }(0, T) \times \partial \Omega,
\end{aligned}
$$

satisfies

$$
(\sigma(T), \mathbf{y}(T))=(\bar{\sigma}(T), \overline{\mathbf{y}}(T)) .
$$

Our goal is to present a positive answer to this control problem under suitable assumptions on the target trajectory $(\bar{\sigma}, \overline{\mathbf{y}})$, and in particular one of hyperbolic nature on the flow corresponding to $\overline{\mathbf{y}}$. Besides, in the 2-dimensional case, our strategy will yield a control acting on some suitable subsets of the boundary which correspond, roughly speaking, to the complement of the part of the boundary in which the scalar product of the target velocity $\overline{\mathbf{y}}$ with the normal vector $\mathbf{n}$ is positive for all time $t \in[0, T]$.

Going further requires some notations. We denote by $L^{2}(\Omega), L^{\infty}(\Omega), H^{r}(\Omega), H_{0}^{r}(\Omega)$ etc. for $r \geq 0$, the usual Lebesgue and Sobolev spaces of scalar functions, and we write in bold the spaces of vector-valued functions: $\mathbf{L}^{2}(\Omega)=$ $\left(L^{2}(\Omega)\right)^{d}, \mathbf{H}^{r}(\Omega)=\left(H^{r}(\Omega)\right)^{d}$, etc. We also define

$$
\mathbf{V}_{0}^{1}(\Omega): \stackrel{\text { def }}{=}\left\{\mathbf{v} \in \mathbf{H}_{0}^{1}(\Omega) \mid \operatorname{div} \mathbf{v}=0 \text { in } \Omega\right\} .
$$

In the following, we will always assume that the target velocity $\overline{\mathbf{y}}$ belongs to $\mathbf{C}^{2}\left(\overline{\Omega_{T}}\right)$. It can thus be extended into a $\mathbf{C}^{2}\left([0, T] \times \mathbb{R}^{d}\right)$ function, still denoted the same for simplicity but not necessarily divergence free outside $\Omega_{T}$. This allows to define the flow $\bar{X}=\bar{X}(t, \tau, x)$ associated to that velocity $\overline{\mathbf{y}}$ :

$$
\forall(t, \tau, x) \in[0, T]^{2} \times \mathbb{R}^{d}, \quad \partial_{t} \bar{X}(t, \tau, x)=\overline{\mathbf{y}}(t, \bar{X}(t, \tau, x)), \quad \bar{X}(\tau, \tau, x)=x .
$$

Thus we define the outgoing subset of $\bar{\Omega}$ for the flow $\bar{X}$ as follows:

$$
\Omega_{\text {out }}^{T}: \stackrel{\text { def }}{=}\left\{x \in \bar{\Omega} \mid \exists t \in(0, T) \text { s.t. } \bar{X}(t, 0, x) \in \mathbb{R}^{d} \backslash \bar{\Omega}\right\} .
$$

One of our main assumptions is the following one:

$$
\bar{\Omega}=\Omega_{\text {out }}^{T} \text {. }
$$

Note that this assumption does not depend on the extension $\overline{\mathbf{y}}$ on $[0, T] \times \mathbb{R}^{d}$ and is intrinsic. This assumption is of hyperbolic nature as it requires the time $T$ to be large enough to guarantee that all the particles that were in $\bar{\Omega}$ at time $t=0$ have been transported by the flow outside $\bar{\Omega}$ in a time strictly smaller than $T$. Of course, this is remanent from the density equation $(1.2)_{(1)}$ in which the density is transported along the flow corresponding to the velocity of the fluid.

As we said, in dimension 2, we will not require the control to be supported on the whole boundary $(0, T) \times \partial \Omega$, but only on some part of it $(0, T) \times \Gamma_{c}$ where $\Gamma_{c}=\partial \Omega \backslash \Gamma_{0}$ and $\Gamma_{0}$ (the part without control) is an open subset of $\partial \Omega$ satisfying the following conditions:

(i). $\quad \Gamma_{0}$ has a finite number of connected components,

(ii). $\quad \inf _{[0, T] \times \overline{\Gamma_{0}}} \overline{\mathbf{y}} \cdot \mathbf{n}>0$. 
Note that the above condition guarantees the existence of $\gamma>0$ such that $\overline{\mathbf{y}}(t, x) \cdot \mathbf{n}(x) \geq \gamma$ for all $(t, x) \in(0, T) \times \Gamma_{0}$. It also implies that $\Gamma_{c}$ is non-empty due to the divergence free condition div $\overline{\mathbf{y}}=0$.

Our main result states as follows:

Theorem 1.1. Let $\Omega$ be a smooth bounded domain of $\mathbb{R}^{2}$. Assume that the target trajectory $(\bar{\sigma}, \overline{\mathbf{y}})$ solution of $(1.1)$ satisfies

$$
(\bar{\sigma}, \overline{\mathbf{y}}) \in C^{2}([0, T] \times \bar{\Omega}) \times \mathbf{C}^{2}([0, T] \times \bar{\Omega}) \quad \text { and } \inf _{[0, T] \times \bar{\Omega}} \bar{\sigma}>0 .
$$

Assume that the condition (1.8) is satisfied for the time $T$.

Then there exists $\varepsilon>0$ such that for all $\left(\rho_{0}, \mathbf{u}_{0}\right) \in L^{\infty}(\Omega) \times \mathbf{V}_{0}^{1}(\Omega)$ satisfying

$$
\left\|\rho_{0}\right\|_{L^{\infty}(\Omega)}+\left\|\mathbf{u}_{0}\right\|_{\mathbf{H}_{0}^{1}(\Omega)} \leq \varepsilon
$$

there exists a controlled trajectory

$$
(\sigma, \mathbf{y}) \in L^{\infty}\left(\Omega_{T}\right) \times H^{1}\left(0, T ; \mathbf{L}^{2}(\Omega)\right) \cap L^{2}\left(0, T ; \mathbf{H}^{2}(\Omega)\right)
$$

solution of (1.2)-(1.4) satisfying the control requirement (1.5).

Besides, if $\Gamma_{0}$ denotes an open subset of the boundary satisfying (1.9), we may further impose $\mathbf{y}=\overline{\mathbf{y}}$ on $(0, T) \times \Gamma_{0}$. In particular, in that case, no boundary condition is imposed on the density on $\Gamma_{0}$.

Actually, we will only prove Theorem 1.1 when $\Gamma_{0} \neq \emptyset$. When $\Gamma_{0}=\emptyset$, the proof is the same as for Theorem 1.2 below dealing with the 3-d case.

Indeed, when the control acts on the whole boundary, Theorem 1.1 can be extended to the 3-dimensional case:

Theorem 1.2. Let $\Omega$ be a smooth bounded domain of $\mathbb{R}^{3}$. Assume that the target trajectory $(\bar{\sigma}, \overline{\mathbf{y}})$ solution of $(1.1)$ satisfies (1.10). Assume that the condition (1.8) is satisfied for the time T.

Then there exists $\varepsilon>0$ such that for all $\left(\rho_{0}, \mathbf{u}_{0}\right) \in L^{\infty}(\Omega) \times \mathbf{V}_{0}^{1}(\Omega)$ satisfying (1.11), there exists a controlled trajectory $(\sigma, \mathbf{y})$ solution of (1.2)-(1.4) and satisfying the control requirement (1.5) and the regularity (1.12).

We refer to Appendix $\mathrm{C}$ for the proof of Theorem 1.2 which can be done similarly as Theorem 1.1 up to some minor changes. Therefore, in the following, except in Appendix C, we will only discuss Theorem 1.1, i.e. the 2-dimensional case.

Strategy of the proof. The proof of Theorem 1.1 is based on a technical fixed-point procedure, and we briefly explain below its general strategy.

Setting

$$
\rho: \stackrel{\text { def }}{=} \sigma-\bar{\sigma}, \quad \mathbf{u}: \stackrel{\text { def }}{=} \mathbf{y}-\overline{\mathbf{y}},
$$

and

$$
\mathbf{f}(\rho, \mathbf{u}): \stackrel{\text { def }}{=}-\rho\left(\partial_{t} \mathbf{u}+((\overline{\mathbf{y}}+\mathbf{u}) \cdot \nabla) \mathbf{u}+(\mathbf{u} \cdot \nabla) \overline{\mathbf{y}}\right)-\bar{\sigma}(\mathbf{u} \cdot \nabla) \mathbf{u}-\rho\left(\partial_{t} \overline{\mathbf{y}}+(\overline{\mathbf{y}} \cdot \nabla) \overline{\mathbf{y}}\right),
$$

Eqs. (1.2)-(1.5) rewrite

$$
\begin{cases}\partial_{t} \rho+(\overline{\mathbf{y}}+\mathbf{u}) \cdot \nabla \rho=-\mathbf{u} \cdot \nabla \bar{\sigma} & \text { in } \Omega_{T}, \\ \bar{\sigma} \partial_{t} \mathbf{u}+\bar{\sigma}(\overline{\mathbf{y}} \cdot \nabla) \mathbf{u}+\bar{\sigma}(\mathbf{u} \cdot \nabla) \overline{\mathbf{y}}-v \Delta \mathbf{u}+\nabla p=\mathbf{f}(\rho, \mathbf{u}) & \text { in } \Omega_{T}, \\ \operatorname{div} \mathbf{u}=0 & \text { in } \Omega_{T}, \\ (\rho(0), \mathbf{u}(0))=\left(\rho_{0}, \mathbf{u}_{0}\right) & \text { in } \Omega,\end{cases}
$$

with the boundary conditions

$$
\mathbf{u}=\mathbf{0} \text { on }(0, T) \times \Gamma_{0},
$$

and with the requirement 


$$
(\rho(T), \mathbf{u}(T))=(0, \mathbf{0}) \quad \text { in } \Omega .
$$

To construct a solution of (1.15)-(1.17), the strategy consists in finding a fixed-point to some mapping $\mathscr{F}_{\left(\rho_{0}, \mathbf{u}_{0}\right)}: \widehat{\mathbf{u}} \mapsto \mathbf{u}$ defined in such a way that $\mathbf{u}=\mathscr{F}_{\left(\rho_{0}, \mathbf{u}_{0}\right)}(\widehat{\mathbf{u}})$ is a suitable solution of:

$$
\begin{cases}\partial_{t} \rho+(\overline{\mathbf{y}}+\widehat{\mathbf{u}}) \cdot \nabla \rho=-\widehat{\mathbf{u}} \cdot \nabla \bar{\sigma} & \text { in } \Omega_{T}, \\ \bar{\sigma} \partial_{t} \mathbf{u}+\bar{\sigma}(\overline{\mathbf{y}} \cdot \nabla) \mathbf{u}+\bar{\sigma}(\mathbf{u} \cdot \nabla) \overline{\mathbf{y}}-v \Delta \mathbf{u}+\nabla p=\mathbf{f}(\rho, \widehat{\mathbf{u}}) & \text { in } \Omega_{T}, \\ \operatorname{div} \mathbf{u}=0 & \text { in } \Omega_{T}, \\ \mathbf{u}=\mathbf{0} & \text { on }(0, T) \times \Gamma_{0}, \\ (\rho(0), \mathbf{u}(0))=\left(\rho_{0}, \mathbf{u}_{0}\right) & \text { in } \Omega, \\ (\rho(T), \mathbf{u}(T))=(0, \mathbf{0}) & \text { in } \Omega .\end{cases}
$$

The mapping $\mathscr{F}_{\left(\rho_{0}, \mathbf{u}_{0}\right)}$ is defined in two steps. First, for a given $\widehat{\mathbf{u}}$, we define $\mathscr{F}_{1}\left(\widehat{\mathbf{u}}, \rho_{0}\right): \stackrel{\text { def }}{=} \rho$, where $\rho$ will be constructed as a suitable solution of the following control problem for the equation of the density:

$$
\begin{cases}\partial_{t} \rho+(\overline{\mathbf{y}}+\widehat{\mathbf{u}}) \cdot \nabla \rho=-\widehat{\mathbf{u}} \cdot \nabla \bar{\sigma} & \text { in } \Omega_{T}, \\ \rho(0)=\rho_{0} & \text { in } \Omega, \\ \rho(T)=0 & \text { in } \Omega .\end{cases}
$$

Then, we define $\mathscr{F}_{2}\left(\mathbf{f}, \mathbf{u}_{0}\right): \stackrel{\text { def }}{=} \mathbf{u}$, where $\mathbf{u}$ is a suitable solution of the following control problem for the equation of the velocity:

$$
\begin{cases}\bar{\sigma} \partial_{t} \mathbf{u}+\bar{\sigma}(\overline{\mathbf{y}} \cdot \nabla) \mathbf{u}+\bar{\sigma}(\mathbf{u} \cdot \nabla) \overline{\mathbf{y}}-v \Delta \mathbf{u}+\nabla p=\mathbf{f} & \text { in } \Omega_{T}, \\ \operatorname{div} \mathbf{u}=0 & \text { in } \Omega_{T}, \\ \mathbf{u}=\mathbf{0} & \text { on }(0, T) \times \Gamma_{0}, \\ \mathbf{u}(0)=\mathbf{u}_{0} & \text { in } \Omega, \\ \mathbf{u}(T)=\mathbf{0} & \text { in } \Omega .\end{cases}
$$

The mapping $\mathscr{F}_{\left(\rho_{0}, \mathbf{u}_{0}\right)}$ is then defined as follows:

$$
\mathscr{F}_{\left(\rho_{0}, \mathbf{u}_{0}\right)}(\widehat{\mathbf{u}}): \stackrel{\text { def }}{=} \mathbf{u}, \quad \text { where } \rho=\mathscr{F}_{1}\left(\widehat{\mathbf{u}}, \rho_{0}\right), \text { and } \mathbf{u}=\mathscr{F}_{2}\left(\mathbf{f}(\rho, \widehat{\mathbf{u}}), \mathbf{u}_{0}\right) .
$$

Hence our strategy decouples the control problem (1.2)-(1.5) into two control problems, (1.19) for the equation of the density, and (1.20) for the equation of the velocity, each of which having different behaviors.

Indeed, on one hand, the control problem (1.20) is of parabolic type, and it will be handled by using global Carleman estimates following the general approach of Fursikov and Imanuvilov [17] for the heat equations: in the case of Navier-Stokes equations, this approach has already been successfully implemented in the works [22,14].

On the other hand, the control problem (1.19) involves a transport equation. This can be easily controlled provided the time $T>0$ is large enough to allow all the particles in $\Omega$ to go outside the domain, i.e. when condition (1.8) is satisfied.

But the problem is that we want the above mapping $\mathscr{F}_{\left(\rho_{0}, \mathbf{u}_{0}\right)}$ to map some convex set into itself. In order to do this, we should be able to get estimates on the above control problems in spaces that behave suitably with respect to both of them. In particular, this will lead us to introduce Carleman weights that follow the dynamics of the transport equation, that is weight functions which are transported by the flow. This strategy then follows the one recently developed in [11] for deriving local exact controllability results for the $1 \mathrm{~d}$ compressible Navier-Stokes equations around constant non-vanishing velocities.

Actually, the Carleman estimates we develop in this article also present the feature of not vanishing at time $t=0$. This allows us to construct a solution $(\rho, \mathbf{u})$ of $(1.15)$ without using any property of the Cauchy problem for the non-homogeneous Navier-Stokes equations.

Related references and comments. To our knowledge, control properties for non-homogeneous Navier-Stokes equations have only been studied in [12], which proves several optimal control results in that context for various cost functions.

For the homogeneous Navier-Stokes equations, the density is assumed to be constant and thus the equations reduce to the equations on the velocity. In that case, several local exact controllability results have been established in $[22,14]$ 
based on parabolic Carleman estimates, see e.g. [17,13]. Later on, several different strategies have been proposed, see for instance $[15,21,24]$. We also point out that these results also use the Carleman estimate derived in [23] for non-homogeneous elliptic problems in order to handle the pressure term.

Let us also quote the work [8] showing the global exact controllability for the 2-d homogeneous Navier-Stokes equations on a manifold without boundary, the work [16] showing the global exact controllability for the 3-d homogeneous Navier-Stokes equations on a torus, and the work [6] focusing on the case of Fourier boundary conditions and showing global approximate controllability in that case. These works actually rely on a similar strategy as the one developed in the context of homogeneous Euler equations [7,20] based on the well-known return method. The case of Boussinesq equations, introducing a coupling between a heat equation and the Navier-Stokes equations, has also been widely studied $[18,16,15]$.

But our problem also involves some transport phenomenon, and therefore also shares some features of the thermoelasticity equations [1], the viscoelasticity models [26,5], and the compressible Navier-Stokes equations [11]. Our approach is actually close to the one developed in [11]. Though, the divergence free condition in the model we consider here requires a specific treatment.

In this article, we will not use any result on the Cauchy problem for (1.2), as our strategy will automatically construct a trajectory $(\sigma, \mathbf{y})$ solving Eqs. (1.2). However, several results are available in the literature. We refer to the work [12] for several results and comments on the Cauchy problem for the non-homogeneous incompressible Navier-Stokes equations and to the references therein.

Let us also note that we will need a precise understanding of the transport equation when transported by a flow entering the domain. More precisely, we will use in an essential way the compactness result in [3, Theorem 4], obtained as a consequence of [2].

We also underline that Theorem 1.1 does not state the uniqueness of the controlled trajectory $(\sigma, \mathbf{y})$. This is due to the lack of regularity for the density $\sigma$ which only belongs to $L^{\infty}\left(\Omega_{T}\right)$, see [9] for the uncontrolled case. In our results, the control set can be reduced to some part of the boundary only in the 2-d case. The reason comes from the fact that, to handle the boundary terms, we use the stream function of the velocity, see Section 2, and that the gradient of this stream function is bounded by the velocity pointwise, which is not true in dimension 3. Nevertheless, the results in [24] seem to indicate that such use of the stream function could be avoided. But this would require significant developments on the Carleman estimate we use, in particular to improve the powers of the Carleman parameters in front of the boundary terms in Theorem 2.4.

Our result also allows the use of non-trivial trajectories. For instance, if $\Gamma_{0}=\emptyset$ and $(\bar{\sigma}, \overline{\mathbf{y}})=(1, \mathbf{0})$, one may consider the trajectory $\left(\bar{\sigma}^{*}(t), \overline{\mathbf{y}}^{*}(t)\right)=(1, \eta(t / T) \mathbf{U})$ for constant vector fields $\mathbf{U}$ and $\eta=\eta(t) \in[0,1]$ a bump function taking value 0 at $t=0$ and $t=1$ and with $\eta=1$ on $[1 / 3,2 / 3]$. Note that $\left(\bar{\sigma}^{*}(t), \overline{\mathbf{y}}^{*}(t)\right)=(1, \mathbf{0})$ at time $t=0$ and at time $t=T$. But for $T>0$ and large $\mathbf{U},\left(\bar{\sigma}^{*}(t), \overline{\mathbf{y}}^{*}(t)\right)$ satisfies (1.8) and all the assumptions of Theorem 1.1, while whatever the time $T>0$ is, the trajectory $(\bar{\sigma}(t), \overline{\mathbf{y}}(t))=(1, \mathbf{0})$ clearly does not satisfy (1.8). This suggests that the geometric condition (1.8) may be avoided in some cases using "return method" type ideas, see e.g. [6,8].

The regularity conditions (1.10) seem strong but are required in our approach. The condition $\overline{\mathbf{y}} \in \mathbf{C}^{2}([0, T] \times \bar{\Omega})$ is used to construct the weight function for our Carleman estimate, thus requiring the $C^{2}$ regularity. On the density, we need at least $\nabla^{\perp} \bar{\sigma} \in W^{1, \infty}\left(0, T ; \mathbf{L}^{\infty}(\Omega)\right)$ to apply Theorem 2.4 to $w$ solution of (2.18).

The geometric condition (1.8) is very likely optimal in general. However, there are some geometric cases of interest in which, though it cannot be satisfied, we expect some results to hold. For instance, when considering a target trajectory $(\bar{\sigma}, \overline{\mathbf{y}})$ corresponding to a constant density $\bar{\sigma}=\mathrm{cst}$, one can easily adapt our proof to show that if the initial perturbation $\left(\rho_{0}, \mathbf{u}_{0}\right)$ satisfies (1.11) and the density $\rho_{0}$ satisfies Supp $\rho_{0} \Subset \Omega_{\text {out }}^{T}$, then there exists a controlled trajectory ( $\sigma, \mathbf{y})$ solution of (1.2)-(1.4) and the control requirement (1.5) (indeed in that case, as $\nabla \bar{\sigma}=0$, the backward density $\rho_{b}$ in (3.7) simply vanishes, so that we can simply take $\rho=\rho_{f}$ in Section 3.2). Another case of interest arises for instance when considering the stabilization of a (non-trivial) Poiseuille flow in a channel. Even thought this flow cannot satisfy the geometric condition (1.8) due to Dirichlet boundary conditions, it is natural to expect that it can be stabilized. But such case requires more work.

One can also ask if our result can be generalized to compressible fluids. Though we expect similar geometric conditions as the one in (1.8) to be needed, the coupling between the density and the fluid velocity is stronger and the question thus requires more work. This issue is currently under investigation.

Outline. This article is organized as follows. Section 2 explains how to solve the control problem (1.20) by the use of Carleman estimates for the Stokes operator. Section 3 shows how to construct a controlled density satisfying 
(1.19) and to derive weighted estimates on it. Section 4 then focuses on the proof of Theorem 1.1 by putting together the arguments developed in Sections 2 and 3. Appendices A and B present some technical proofs. Appendix C proves Theorem 1.2.

\section{Controlling the velocity}

This section is dedicated to the construction of a solution of (1.20).

\subsection{Statement of the result}

In order to solve the control problem (1.20), we will consider (1.20) in an extended domain $\mathcal{O}$ as follows: $\mathcal{O}$ is a smooth bounded domain of $\mathbb{R}^{2}$ satisfying

$$
\Omega \subset \mathcal{O}, \quad \partial \mathcal{O} \text { is of class } C^{2}, \quad \partial \mathcal{O} \cap \partial \Omega \supset \Gamma_{0} .
$$

We then extend $(\bar{\sigma}, \overline{\mathbf{y}})$ on $[0, T] \times \overline{\mathcal{O}}$, still denoted the same for simplicity, such that

$$
(\bar{\sigma}, \overline{\mathbf{y}}) \in C^{2}([0, T] \times \overline{\mathcal{O}}) \times \mathbf{C}^{2}([0, T] \times \overline{\mathcal{O}}) \quad \text { and } \inf _{[0, T] \times \overline{\mathcal{O}}} \bar{\sigma}(t, x)>0 .
$$

Remark that this is possible due to the assumption (1.10). As $\mathbf{u}_{0} \in \mathbf{V}_{0}^{1}(\Omega)$, extending it by zero outside $\Omega$, we get an extension, still denoted the same, such that

$$
\mathbf{u}_{0} \in \mathbf{H}_{0}^{1}(\mathcal{O}) \quad \text { and } \quad \operatorname{div} \mathbf{u}_{0}=0 \quad \text { in } \mathcal{O} .
$$

By also extending $\mathbf{f}$ by zero outside $\Omega$ and setting $\mathcal{O}_{T}=(0, T) \times \mathcal{O}, \Gamma_{T}=(0, T) \times \partial \mathcal{O}$ we then consider the following system

$$
\begin{cases}\bar{\sigma}\left(\partial_{t} \mathbf{u}+(\overline{\mathbf{y}} \cdot \nabla) \mathbf{u}+(\mathbf{u} \cdot \nabla) \overline{\mathbf{y}}\right)-v \Delta \mathbf{u}+\nabla p=\mathbf{f}+\mathbf{h} 1_{\mathcal{O} \backslash \bar{\Omega}} & \text { in } \mathcal{O}_{T}, \\ \operatorname{div} \mathbf{u}=0 & \text { in } \mathcal{O}_{T}, \\ \mathbf{u}=\mathbf{0} & \text { on } \Gamma_{T}, \\ \mathbf{u}(0)=\mathbf{u}_{0} & \text { in } \mathcal{O} .\end{cases}
$$

Here, $1_{\mathcal{O} \backslash \bar{\Omega}}$ is the characteristic function of $\mathcal{O} \backslash \bar{\Omega}$ and $\mathbf{h} \in \mathbf{L}^{2}\left(\mathcal{O}_{T}\right)$ is a control function. Note that the presence of $1_{\mathcal{O} \backslash \bar{\Omega}}$ in (2.4) implies that the action of the control is supported in $\mathcal{O} \backslash \bar{\Omega}$.

We thus intend to solve the following control problem: Given $\mathbf{u}_{0} \in \mathbf{H}_{0}^{1}(\mathcal{O})$ satisfying (2.3) and a source term $\mathbf{f}$ in some suitable space, find a control function $\mathbf{h} \in \mathbf{L}^{2}\left(\mathcal{O}_{T}\right)$ such that the solution $\mathbf{u}$ of (2.4) satisfies

$$
\mathbf{u}(T)=0 \quad \text { in } \mathcal{O} .
$$

Indeed, if we are able to solve this control problem, the restriction of the solution $\mathbf{u}$ to $\Omega$ would yield a solution of the control problem (1.20). In order to solve the control problem (2.4)-(2.5), as it is classical by now, we are going to establish a suitable observability estimate for the adjoint problem

$$
\begin{cases}-\partial_{t}(\bar{\sigma} \mathbf{v})-D(\bar{\sigma} \mathbf{v}) \overline{\mathbf{y}}-\bar{\sigma} \mathbf{v} \operatorname{div} \overline{\mathbf{y}}-v \Delta \mathbf{v}+\nabla p=\mathbf{g} & \text { in } \mathcal{O}_{T}, \\ \operatorname{div} \mathbf{v}=0 & \text { in } \mathcal{O}_{T}, \\ \mathbf{v}=\mathbf{0} & \text { on } \Gamma_{T},\end{cases}
$$

where $D \mathbf{v}:=\nabla \mathbf{v}+{ }^{t} \nabla \mathbf{v}$ is the symmetrized gradient. At this step, note that, though $\operatorname{div} \overline{\mathbf{y}}$ vanishes in the set $(0, T) \times \Omega$, there is no reason to further assume that it vanishes in $\mathcal{O}_{T}$, as we do not assume that the domain $\Omega$ is simply connected.

To state our result precisely, let us introduce the weight functions we will use in the Carleman estimate. We assume that we have a function $\tilde{\psi}=\tilde{\psi}(t, x) \in C^{2}\left(\overline{\mathcal{O}_{T}}\right)$ such that

$$
\tilde{\psi}: \stackrel{\text { def }}{=} \tilde{\psi}(t, x) \quad \text { such that } \begin{cases}\forall(t, x) \in \overline{\mathcal{O}_{T}}, & \tilde{\psi}(t, x) \in[0,1], \\ \forall(t, x) \in \Gamma_{T}, & \partial_{\mathbf{n}} \tilde{\psi}(t, x) \leq 0, \\ \forall t \in[0, T], & \tilde{\psi}(t)_{\mid \partial \mathcal{O}} \text { is constant, } \\ \forall t \in[0, T], & \inf _{\mathcal{O}} \tilde{\psi}(t, \cdot)=\tilde{\psi}(t)_{\mid \partial \mathcal{O}} .\end{cases}
$$


We also assume the existence of two open subsets $\tilde{\omega}_{T} \Subset \omega_{T}$ of $[0, T] \times(\mathcal{O} \backslash \bar{\Omega})$ (here and in the following, the symbol $\Subset$ means that there exists a compact set $K_{T}$ of $[0, T] \times(\mathcal{O} \backslash \bar{\Omega})$ such that $\left.\tilde{\omega}_{T} \subset K_{T} \subset \omega_{T}\right)$ and a constant $\alpha>0$ such that

$$
\inf _{\mathcal{O}_{T} \backslash \tilde{\omega}_{T}}\{|\nabla \tilde{\psi}|\} \geq \alpha>0 .
$$

For $m \geq 1$, we set

$$
\psi(t, x): \stackrel{\text { def }}{=} \tilde{\psi}(t, x)+6 m .
$$

We then set $T_{0}>0$ and $T_{1}>0$ such that $T_{1} \leq 1 / 4$ and $T_{0}+2 T_{1}<T$ and choose a weight function in time $\theta_{m, \mu}(t)$ depending on the parameters $m \geq 1$ and $\mu \geq 2$ defined by

$$
\theta_{m, \mu}: \stackrel{\text { def }}{=} \theta_{m, \mu}(t) \quad \text { such that } \quad\left\{\begin{array}{l}
\forall t \in\left[0, T_{0}\right], \quad \theta_{m, \mu}(t)=1+\left(1-\frac{t}{T_{0}}\right)^{\mu}, \\
\forall t \in\left[T_{0}, T-2 T_{1}\right], \quad \theta_{m, \mu}(t)=1, \\
\forall t \in\left[T-T_{1}, T\right), \quad \theta_{m, \mu}(t)=\frac{1}{(T-t)^{m}}, \\
\theta_{m, \mu} \text { is increasing on }\left[T-2 T_{1}, T-T_{1}\right], \\
\theta_{m, \mu} \in C^{2}([0, T)) .
\end{array}\right.
$$

For simplicity of notations in the following we omit the dependence on $m$ and $\mu$ and we simply write $\theta: \stackrel{\text { def }}{=} \theta_{m, \mu}$. We will then take the following weight functions $\varphi=\varphi(t, x)$ and $\xi=\xi(t, x)$ :

$$
\varphi(t, x): \stackrel{\text { def }}{=} \theta(t)\left(\lambda e^{6 \lambda(m+1)}-\exp (\lambda \psi(t, x))\right), \quad \xi(t, x): \stackrel{\text { def }}{=} \theta(t) \exp (\lambda \psi(t, x)),
$$

where $s, \lambda$ are positive parameters with $s \geq 1, \lambda \geq 1$ and $\mu$ is chosen as

$$
\mu=s \lambda^{2} e^{\lambda(6 m-4)}
$$

which is always bigger than 2 , thus being compatible with the condition $\theta \in C^{2}([0, T))$. Note that the weight functions $\varphi$ and $\xi$, depend on $s, \lambda, m$, and should rather be denoted by $\varphi_{s, \lambda, m}$, resp. $\xi_{s, \lambda, m}$, but we drop these indexes for simplicity of notations.

Remark that, due to the definition of $\psi$ in (2.9) and the conditions (2.7), we have, for all $\lambda \geq 1$ and $(t, x) \in \mathcal{O}_{T}$,

$$
\frac{3}{4} \theta(t) \lambda e^{6 \lambda(m+1)} \leq \varphi(t, x) \leq \theta(t) \lambda e^{6 \lambda(m+1)} .
$$

Finally, we introduce

$$
\begin{aligned}
& \widehat{\varphi}(t): \stackrel{\text { def }}{=} \min _{x \in \overline{\mathcal{O}}} \varphi(t, x), \varphi^{*}(t): \stackrel{\text { def }}{=} \max _{x \in \overline{\mathcal{O}}} \varphi(t, x)=\varphi_{\mid \partial \mathcal{O}}(t), \\
& \widehat{\xi}(t): \stackrel{\text { def }}{=} \max _{x \in \overline{\mathcal{O}}} \xi(t, x), \quad \xi^{*}(t): \stackrel{\text { def }}{=} \min _{x \in \overline{\mathcal{O}}} \xi(t, x)=\xi_{\mid \partial \mathcal{O}}(t) .
\end{aligned}
$$

Using these weight functions, we prove the following Carleman estimate for the Stokes system (2.6):

Theorem 2.1. Assume that $\mathcal{O}$ is a smooth bounded domain extending $\Omega$ as in (2.1), let $\omega, \tilde{\omega}$ be two subdomains of $\mathcal{O} \backslash \bar{\Omega}$ such that $\tilde{\omega} \Subset \omega$ and set $\omega_{T}=[0, T] \times \omega$ and $\tilde{\omega}_{T}=[0, T] \times \tilde{\omega}$.

Let $\tilde{\psi}$ as in (2.7)-(2.8) and $\psi, \theta, \varphi$, $\xi$ as in (2.9)-(2.11).

Then, for $m \geq 5$, there exist some constants $s_{0} \geq 1, \lambda_{0} \geq 1$ and $C>0$ such that for all smooth solution $\mathbf{v}$ of (2.6) with source term $\mathbf{g} \in \mathbf{L}^{2}\left(\mathcal{O}_{T}\right)$, for all $s \geq s_{0}$ and $\lambda \geq \lambda_{0}$,

$$
\begin{aligned}
& s^{1 / 2} \lambda^{-1 / 2} \int_{\mathcal{O}}\left(\xi^{*}\right)^{4-2 / m}|\mathbf{v}(0, \cdot)|^{2} e^{-2 s \varphi^{*}(0)}+s \lambda^{2} \iint_{\mathcal{O}_{T}} \xi^{4}|\mathbf{v}|^{2} e^{-2 s \varphi} \\
& +s^{-1} \iint_{\mathcal{O}_{T}} \xi^{2}|\nabla \mathbf{v}|^{2} e^{-2 s \varphi}+s^{1 / 2} \lambda^{-1 / 2} \int_{0}^{T}\left(\xi^{*}\right)^{4-2 / m} e^{-2 s \varphi^{*}}\|\mathbf{v}\|_{\mathbf{H}^{1}(\mathcal{O})}^{2}
\end{aligned}
$$




$$
\leq C\left(s^{5 / 2} \lambda^{2} \iint_{\omega_{T}} \widehat{\xi}^{6}|\mathbf{v}|^{2} e^{2 s \varphi^{*}-4 s \widehat{\varphi}}+s^{1 / 2} \lambda^{-1 / 2} \iint_{\mathcal{O}_{T}}(\xi)^{4-2 / m}|\mathbf{g}|^{2} e^{-2 s \varphi}\right) .
$$

Remark 2.2. Estimate (2.16) can be completed with higher norms on $\mathbf{v}$ in the left-hand side of (2.16). Namely, looking carefully at the proof of Theorem 2.1, in particular in (2.52), we can add in the left-hand side of (2.16) the terms

$$
\begin{aligned}
& s^{-1 / 2} \lambda^{-3 / 2}\left\|\left(\xi^{*}\right)^{3 / 2-3 /(2 m)} \mathbf{v} e^{-s \varphi^{*}(t)}\right\|_{L^{2}\left(0, T ; \mathbf{H}^{2}(\mathcal{O})\right)}^{2} \\
& \quad+s^{-1 / 2} \lambda^{-3 / 2}\left\|\left(\xi^{*}\right)^{3 / 2-3 /(2 m)} \nabla p e^{-s \varphi^{*}(t)}\right\|_{L^{2}\left(0, T ; L^{2}(\mathcal{O})\right)}^{2}
\end{aligned}
$$

and also, thanks to maximal regularity results for the equation satisfied by the pair $s^{-3 / 2} \lambda^{-2}\left(\xi^{*}\right)^{-1 /(3 m)} e^{-s \varphi^{*}(t)}(\mathbf{v}, p)$,

$$
s^{-3} \lambda^{-4}\left\|\left(\xi^{*}\right)^{-1 /(3 m)} \mathbf{v} e^{-s \varphi^{*}(t)}\right\|_{H^{1}\left(0, T ; \mathbf{L}^{2}(\mathcal{O})\right)}^{2} .
$$

The proof of Theorem 2.1 is done in Sections 2.2 and 2.3. We are first going to prove a slightly improved version of the Carleman estimates (2.16) for solutions $\mathbf{v}$ of the simplified version of the adjoint problem (2.6):

$$
\begin{cases}-\bar{\sigma} \partial_{t} \mathbf{v}-v \Delta \mathbf{v}+\nabla p=\mathbf{g} & \text { in } \mathcal{O}_{T} \\ \operatorname{div} \mathbf{v}=0 & \text { in } \mathcal{O}_{T} \\ \mathbf{v}=0 & \text { on } \Gamma_{T}\end{cases}
$$

Our approach then consists first in taking the curl of Eq. (2.17) and consider the equation of $w=$ curl v:

$$
-\bar{\sigma} \partial_{t} w-v \Delta w=\operatorname{curl} \mathbf{g}+\partial_{t} \mathbf{v} \cdot \nabla^{\perp} \bar{\sigma} \quad \text { in } \mathcal{O}_{T} .
$$

Thus, in Section 2.2, we derive estimates on $w$ solution of (2.18) in terms of the right hand side of the equation of (2.18) and the boundary terms. It turns out that the boundary conditions and source terms strongly depend on $\mathbf{v}$ itself. Hence in Section 2.3, we explain how to estimate $\mathbf{v}$ in terms of $w$ by using the stream function $\zeta$ associated to $\mathbf{u}$, which is given by

$$
\Delta \zeta(t)=w(t) \quad \text { in } \mathcal{O}_{T} \quad \text { and } \quad \zeta(t)=c_{i}(t) \quad \text { on }[0, T] \times \gamma_{i} \text { for } i=1, \ldots, K,
$$

where $\left\{\gamma_{i}, i=1, \ldots, K\right\}$ is the family of connected components of $\partial \mathcal{O}$ and $c_{i}(t), i=1, \ldots, K$ are some constants characterizing $\zeta(t)$ which are chosen such that, for some Lipschitz subdomain $\widehat{\omega}$ of $\mathcal{O} \backslash \bar{\Omega}$ satisfying $\tilde{\omega} \Subset \widehat{\omega} \Subset \omega$,

$$
\int_{\widehat{\omega}} \zeta(t)=0
$$

Among the new features of the Carleman estimate of Theorem 2.1 with respect to those in the literature, let us point out the following facts:

- The weight function in time $\theta_{m, \mu}$ in (2.10) does not blow up as the time $t$ goes to 0 . However, our proof requires a strong convexity property close to $t=0$, tuned by the choice of the parameter $\mu$ in (2.10) as a suitable function of the parameters $s$ and $\lambda$, see (2.12).

- The weight function $\psi$ depends on both the time and space variables. As we shall explain, this is not a big issue as long as we guarantee that for all $t \in[0, T], \psi(t)$ is constant on the boundary $\partial \mathcal{O}$, thus allowing to apply the Carleman inequality of [23] for elliptic equations.

Based on Theorem 2.1, following standard duality arguments, we prove the following control result:

Theorem 2.3. Within the setting and assumptions of Theorem 2.1, there exists a constant $C>0$ such that for all $s \geq s_{0}$ and $\lambda \geq \lambda_{0}$, if $\mathbf{u}_{0}$ verifies (2.3) and $\mathbf{f} \in \mathbf{L}^{2}\left(\mathcal{O}_{T}\right)$ satisfies

$$
\iint_{\mathcal{O}_{T}} \xi^{-4}|\mathbf{f}|^{2} e^{2 s \varphi}<\infty
$$


there exists a control function $\mathbf{h} \in \mathbf{L}^{2}\left(\mathcal{O}_{T}\right)$ supported in $\omega_{T}$ and a controlled trajectory $\mathbf{u} \in \mathbf{L}^{2}\left(\mathcal{O}_{T}\right)$ such that $\mathbf{u}$ solves the control problem (2.4)-(2.5) and (u, h) satisfies the estimate

$$
\begin{aligned}
& \left\|e^{\frac{3}{4} s \varphi^{*}} \mathbf{u}\right\|_{L^{2}\left(\mathbf{H}^{2}\right) \cap H^{1}\left(\mathbf{L}^{2}\right)}^{2}+s^{1 / 2} \lambda^{5 / 2} \iint_{\mathcal{O}_{T}} \xi^{2 / m-4}|\mathbf{u}|^{2} e^{2 s \varphi}+s^{-3 / 2} \iint_{\omega_{T}} \widehat{\xi}^{-6}|\mathbf{h}|^{2} e^{4 s \widehat{\varphi}-2 s \varphi^{*}} \\
& \quad \leq C\left(\iint_{\mathcal{O}_{T}} \xi^{-4}|\mathbf{f}|^{2} e^{2 s \varphi}+e^{\frac{5}{2} s \varphi^{*}(0, \cdot)}\left\|\mathbf{u}_{0}\right\|_{\mathbf{H}_{0}^{1}(\mathcal{O})}^{2}\right) .
\end{aligned}
$$

The proof of Theorem 2.3 is given in Section 2.4.

\subsection{Carleman estimates for the heat equation}

The goal of this section is to show the following estimate:

Theorem 2.4. Let $\widehat{\omega_{T}}$ be an open subset of $\mathcal{O}_{T}$ satisfying $\tilde{\omega}_{T} \Subset \widehat{\omega_{T}}$ and let $\tilde{\psi}$ as in (2.7)-(2.8) and $\psi, \theta, \varphi, \xi$ as in (2.9)-(2.11).

For all $M>0$, there exist constants $C>0, s_{0}$ and $\lambda_{0}$ such that for all $s \geq s_{0}$ and $\lambda \geq \lambda_{0}$, for all smooth functions w in $\overline{\mathcal{O}_{T}}$, such that

$$
-\bar{\sigma} \partial_{t} w-v \Delta w=a_{0} w+A_{1} \cdot \nabla w+g_{0}+\sum_{i=1}^{d} b_{i} \partial_{i} g_{i}+b_{d+1} \partial_{t} g_{d+1} \quad \text { in } \mathcal{O}_{T},
$$

with $a_{0} \in L^{\infty}\left(\mathcal{O}_{T}\right), A_{1} \in L^{\infty}\left(0, T ; \mathbf{W}^{1, \infty}(\mathcal{O})\right), g_{0}, g_{i} \in L^{2}\left(\mathcal{O}_{T}\right)$, and coefficients $b_{i} \in L^{\infty}\left(0, T ; W^{1, \infty}(\mathcal{O})\right), b_{d+1} \in$ $W^{1, \infty}\left(0, T ; L^{\infty}(\mathcal{O})\right)$ satisfying

$$
\left\|a_{0}\right\|_{L^{\infty}\left(\mathcal{O}_{T}\right)}+\left\|A_{1}\right\|_{L^{\infty}\left(0, T ; \mathbf{W}^{1, \infty}(\mathcal{O})\right)}+\sum_{i=1}^{d}\left\|b_{i}\right\|_{L^{\infty}\left(0, T ; W^{1, \infty}(\mathcal{O})\right)}+\left\|b_{d+1}\right\|_{W^{1, \infty}\left(0, T ; L^{\infty}(\mathcal{O})\right)} \leq M,
$$

we have

$$
\begin{aligned}
s^{3} \lambda^{4} \iint_{\mathcal{O}_{T}} \xi^{3}|w|^{2} e^{-2 s \varphi} \leq & C \iint_{\mathcal{O}_{T}}\left|g_{0}\right|^{2} e^{-2 s \varphi}+C s^{2} \lambda^{2} \iint_{\mathcal{O}_{T}} \xi^{2}\left(\sum_{i=1}^{d}\left|g_{i}\right|^{2}\right) e^{-2 s \varphi}+C s^{4} \lambda^{4} \iint_{\mathcal{O}_{T}} \xi^{4}\left|g_{d+1}\right|^{2} e^{-2 s \varphi} \\
& +C s^{3} \lambda^{3} \int_{\Gamma_{T}} \xi^{3}|w|^{2} e^{-2 s \varphi}+C s^{3} \lambda^{4} \iint_{\widehat{\omega_{T}}} \xi^{3}|w|^{2} e^{-2 s \varphi} .
\end{aligned}
$$

The proof of Theorem 2.4 is long and is divided in three steps:

1. a Carleman estimate for the heat equation with homogeneous boundary conditions and source terms in $L^{2}\left(\mathcal{O}_{T}\right)$; see Theorem 2.5;

2. energy estimates on controlled trajectories of a heat equation with a source term in $L^{2}\left(\mathcal{O}_{T}\right)$; see Theorem 2.6;

3. a duality argument.

This proof is inspired by the ones in [25], see also [13]. Below, we only state Theorems 2.5-2.6, whose proofs are postponed to the appendix. Let us also emphasize that Theorems 2.4-2.6 hold in any dimension $d$.

Proof of Theorem 2.4. As said above, the proof is done in three steps.

An $L^{2}$-Carleman estimate. The first result is the following $L^{2}$-Carleman estimate for the heat equation:

Theorem 2.5. Assume the setting of Theorem 2.4. For all $m \geq 1$, there exist constants $C_{0}>0, s_{0} \geq 1$ and $\lambda_{0} \geq 1$ such that for all smooth functions $z$ on $\overline{\mathcal{O}_{T}}$ satisfying $z=0$ on $\Gamma_{T}$, for all $s \geq s_{0}, \lambda \geq \lambda_{0}$, we have 


$$
\begin{aligned}
& \int_{\mathcal{O}}|\nabla z(0)|^{2} e^{-2 s \varphi(0)}+s^{2} \lambda^{3} e^{2 \lambda(6 m+1)} \int_{\mathcal{O}}|z(0)|^{2} e^{-2 s \varphi(0)}+s \lambda^{2} \iint_{\mathcal{O}_{T}} \xi|\nabla z|^{2} e^{-2 s \varphi}+s^{3} \lambda^{4} \iint_{\mathcal{O}_{T}} \xi^{3}|z|^{2} e^{-2 s \varphi} \\
& \quad \leq C_{0} \iint_{\mathcal{O}_{T}}\left|\left(-\bar{\sigma} \partial_{t}-v \Delta\right) z\right|^{2} e^{-2 s \varphi}+C_{0} s^{3} \lambda^{4} \iint_{\widehat{\omega_{T}}} \xi^{3}|z|^{2} e^{-2 s \varphi} .
\end{aligned}
$$

The proof of Theorem 2.5 is given in Section A.1. It is rather classical except for the weight function $\varphi$, which does not blow up as $t \rightarrow 0$ and for the weight function $\psi$ which depends on both time and space variables. This introduces in the proof of Theorem 2.5 several new technical issues, though our proof follows the lines of [17].

Estimates on a control problem. We then analyze the following control problem: for $f \in L^{2}\left(\mathcal{O}_{T}\right)$, find a control function $h \in L^{2}\left(\widehat{\omega_{T}}\right)$ such that the solution $y$ of

$$
\begin{cases}\partial_{t}(\bar{\sigma} y)-v \Delta y=f+h 1_{\widehat{\omega_{T}}}, & \text { in } \mathcal{O}_{T}, \\ y=0, & \text { on } \Gamma_{T}, \\ y(0, \cdot)=0, & \text { in } \mathcal{O},\end{cases}
$$

solves the control problem:

$$
y(T, \cdot)=0, \quad \text { in } \mathcal{O} .
$$

We claim the following result:

Theorem 2.6. Assume the setting of Theorem 2.4. For all $m \geq 1$, there exist positive constants $C>0, s_{0} \geq 1$ and $\lambda_{0} \geq 1$ such that for all $s \geq s_{0}$ and $\lambda \geq \lambda_{0}$, for all $f$ satisfying

$$
\iint_{\mathcal{O}_{T}} \xi^{-3}|f|^{2} e^{2 s \varphi}<\infty
$$

there exists a solution $(Y, H)$ of the control problem (2.26)-(2.27) which furthermore satisfies the following estimate:

$$
\begin{aligned}
& s^{3} \lambda^{4} \iint_{\mathcal{O}_{T}}|Y|^{2} e^{2 s \varphi}+\iint_{\widehat{\omega_{T}}} \xi^{-3}|H|^{2} e^{2 s \varphi}+s \lambda^{2} \iint_{\mathcal{O}_{T}} \xi^{-2}|\nabla Y|^{2} e^{2 s \varphi} \\
& +\frac{1}{s} \iint_{\mathcal{O}_{T}} \xi^{-4}\left(\left|\partial_{t} Y\right|^{2}+|\Delta Y|^{2}\right) e^{2 s \varphi}+\lambda \int_{\Gamma_{T}} \xi^{-3}\left|\partial_{\mathbf{n}} Y\right|^{2} e^{2 s \varphi} \leq C \iint_{\mathcal{O}_{T}} \xi^{-3}|f|^{2} e^{2 s \varphi} .
\end{aligned}
$$

The proof of Theorem 2.6 is given in Section A.2. Again, the proof is rather classical and is based on the duality between the Carleman estimates, which are weighted observability estimates, and controllability, and then on energy estimates. Note however that these energy estimates have to be derived using the weight functions defined in (2.7)-(2.11), and this introduces some novelties in the computations.

A duality argument. The proof of Theorem 2.4 then relies upon the estimate (2.29) on the solution $(Y, H)$ of the control problem (2.26)-(2.27) for $f=\xi^{3} w e^{-2 s \varphi}$. Indeed, if $(Y, H)$ solves (2.26)-(2.27) for some $f$ satisfying (2.28), multiplying the equation satisfied by $Y$ by $w$, we obtain

$$
\begin{aligned}
& \iint_{\mathcal{O}_{T}} w\left(f+H 1_{\widehat{\omega_{T}}}\right)+\int_{\Gamma_{T}} w \partial_{\mathbf{n}} Y \\
& \quad=\iint_{\mathcal{O}_{T}}\left(a_{0} w Y-w \operatorname{div}\left(A_{1} Y\right)+g_{0} Y-\sum_{i=1}^{n} g_{i} \partial_{i}\left(b_{i} Y\right)-g_{n+1} \partial_{t}\left(b_{n+1} Y\right)\right) .
\end{aligned}
$$

In particular, as $f=\xi^{3} w e^{-2 s \varphi}$ satisfies

$$
\iint_{\mathcal{O}_{T}} \xi^{-3}|f|^{2} e^{2 s \varphi}=\iint_{\mathcal{O}_{T}} \xi^{3}|w|^{2} e^{-2 s \varphi},
$$


according to (2.29) we can construct $(Y, H)$ solution of

$$
\begin{cases}\partial_{t}(\bar{\sigma} Y)-v \Delta Y=\xi^{3} w e^{-2 s \varphi}+H 1_{\widehat{\omega_{T}}}, & \text { in } \mathcal{O}_{T}, \\ Y=0, & \text { on } \Gamma_{T}, \\ Y(0, \cdot)=0, & \text { in } \mathcal{O}, \\ Y(T, \cdot)=0, & \text { in } \mathcal{O},\end{cases}
$$

for which we have the estimate:

$$
\begin{aligned}
& s^{3} \lambda^{4} \iint_{\mathcal{O}_{T}}|Y|^{2} e^{2 s \varphi}+\iint_{\widehat{\omega}_{T}} \xi^{-3}|H|^{2} e^{2 s \varphi}+s \lambda^{2} \iint_{\mathcal{O}_{T}} \xi^{-2}|\nabla Y|^{2} e^{2 s \varphi} \\
& \quad+\frac{1}{s} \iint_{\mathcal{O}_{T}} \xi^{-4}\left(\left|\partial_{t} Y\right|^{2}+|\nabla Y|^{2}\right) e^{2 s \varphi}+\lambda \int_{\Gamma_{T}} \xi^{-3}\left|\partial_{\mathbf{n}} Y\right|^{2} e^{2 s \varphi} \leq C \iint_{\mathcal{O}_{T}} \xi^{3}|w|^{2} e^{-2 s \varphi} .
\end{aligned}
$$

Using then the identity (2.30), we infer

$$
\begin{aligned}
& \iint_{\mathcal{O}_{T}} \xi^{3}|w|^{2} e^{-2 s \varphi} \\
& \leq C\left(\frac{1}{s \lambda^{2}} \iint_{\mathcal{O}_{T}} \xi^{2}|w|^{2} e^{-2 s \varphi}\right)^{1 / 2}\left(s \lambda^{2} \iint \xi^{-2}\left(|Y|^{2}+|\nabla Y|^{2}\right) e^{2 s \varphi}\right)^{1 / 2} \\
&+C\left(\frac{1}{s^{3} \lambda^{4}} \iint_{\mathcal{O}_{T}}\left|g_{0}\right|^{2} e^{-2 s \varphi}\right)^{1 / 2}\left(s^{3} \lambda^{4} \iint_{\mathcal{O}_{T}}|Y|^{2} e^{2 s \varphi}\right)^{1 / 2} \\
&+C\left(\frac{1}{s \lambda^{2}} \iint_{\mathcal{O}_{T}} \xi^{2}\left(\sum_{i=1}^{d}\left|g_{i}\right|^{2}\right) e^{-2 s \varphi}\right)^{1 / 2}\left(s \lambda^{2} \iint_{\mathcal{O}_{T}} \xi^{-2}\left(|Y|^{2}+|\nabla Y|^{2}\right) e^{2 s \varphi}\right)^{1 / 2} \\
&+C\left(s \iint_{\mathcal{O}_{T}} \xi^{4}\left|g_{d+1}\right|^{2} e^{-2 s \varphi}\right)^{1 / 2}\left(\frac{1}{s} \iint_{\mathcal{O}_{T}} \xi^{-4}\left(|Y|^{2}+\left|\partial_{t} Y\right|^{2}\right) e^{2 s \varphi}\right)^{1 / 2} \\
&+C\left(\frac{1}{\lambda} \int_{\Gamma_{T}} \xi^{3}|w|^{2} e^{-2 s \varphi}\right)^{1 / 2}\left(\lambda \int \xi_{\Gamma_{T}}{ }^{-3}\left|\partial_{\mathbf{n}} Y\right|^{2} e^{2 s \varphi}\right)^{1 / 2} \\
&+C\left(\iint_{\omega_{T}} \xi^{3}|w|^{2} e^{-2 s \varphi}\right)^{1 / 2}\left(\iint_{\omega_{T}} \xi^{-3}|H|^{2} e^{2 s \varphi}\right)^{1 / 2},
\end{aligned}
$$

which immediately yields the claimed result by (2.32).

\subsection{Proof of Theorem 2.1}

This section aims at proving Theorem 2.1. This will be done in two steps.

We first prove the following Carleman estimate for $\mathbf{v}$ solution of (2.17):

Theorem 2.7. Within the setting and assumptions of Theorem 2.1, for any $m \geq 5$, there exist some constants $s_{0} \geq 1$, $\lambda_{0} \geq 1$ and $C>0$ such that for all solution $\mathbf{v}$ of (2.17) with source term $\mathbf{g} \in \mathbf{L}^{2}\left(\mathcal{O}_{T}\right)$, for all $s \geq s_{0}$ and $\lambda \geq \lambda_{0}$,

$$
s^{1 / 2} \lambda^{-1 / 2} \int_{\mathcal{O}}\left(\xi^{*}\right)^{4-2 / m}|\mathbf{v}(0, \cdot)|^{2} e^{-2 s \varphi^{*}(0)}+s \lambda^{2} \iint_{\mathcal{O}_{T}} \xi^{4}|\mathbf{v}|^{2} e^{-2 s \varphi}
$$




$$
\begin{aligned}
& +s^{1 / 2} \lambda^{-1 / 2} \int_{0}^{T}\left(\xi^{*}\right)^{4-2 / m} e^{-2 s \varphi^{*}}\|\mathbf{v}\|_{\mathbf{H}^{1}(\mathcal{O})}^{2}+\iint_{\mathcal{O}_{T}} \xi^{3}|\operatorname{curl} \mathbf{v}|^{2} e^{-2 s \varphi}+s^{-1} \iint_{\mathcal{O}_{T}} \xi^{2}|\nabla \mathbf{v}|^{2} e^{-2 s \varphi} \\
\leq & C\left(s^{5 / 2} \lambda^{2} \iint_{\omega_{T}} \widehat{\xi}^{6}|\mathbf{v}|^{2} e^{2 s \varphi^{*}-4 s \widehat{\varphi}}+s^{-1} \lambda^{-2} \iint_{\mathcal{O}_{T}} \xi^{2}|\mathbf{g}|^{2} e^{-2 s \varphi}\right. \\
& \left.+s^{1 / 2} \lambda^{-1 / 2} \int_{0}^{T}\left(\xi^{*}\right)^{4-2 / m} e^{-2 s \varphi^{*}}\|\mathbf{g}\|_{\mathbf{H}^{-1}(\mathcal{O})}^{2}+s^{-1 / 2} \lambda^{-3 / 2} \iint_{\mathcal{O}_{T}}\left(\xi^{*}\right)^{3-3 / m}|\mathbf{g}|^{2} e^{-2 s \varphi^{*}}\right) .
\end{aligned}
$$

The proof of Theorem 2.7 is done below in Section 2.3.1. In Section 2.3.2 we then explain how Theorem 2.7 implies Theorem 2.1 .

\subsubsection{Proof of Theorem 2.7}

Let $\mathbf{v}$ be a solution of (2.17) with source term $\mathbf{g}$. As $w=\operatorname{curl} \mathbf{v}$ satisfies (2.18), the Carleman estimate (2.24) applies to $w$ : for all $s \geq s_{0}$ and $\lambda \geq \lambda_{0}$,

$$
\begin{aligned}
\iint_{\mathcal{O}_{T}} \xi^{3}|w|^{2} e^{-2 s \varphi} \leq & C\left(\iint_{\omega_{T}} \xi^{3}|w|^{2} e^{-2 s \varphi}+s \iint_{\mathcal{O}_{T}} \xi^{4}|\mathbf{v}|^{2} e^{-2 s \varphi}\right. \\
& \left.+\lambda^{-1} \int_{\Gamma_{T}} \xi^{3}|w|^{2} e^{-2 s \varphi}+s^{-1} \lambda^{-2} \iint_{\mathcal{O}_{T}} \xi^{2}|\mathbf{g}|^{2} e^{-2 s \varphi}\right) .
\end{aligned}
$$

Here and in the following $\widehat{\omega}_{T}=[0, T] \times \widehat{\omega}$ where $\widehat{\omega}$ is a Lipschitz subdomain $\mathcal{O} \backslash \bar{\Omega}$ such that $\widetilde{\omega} \Subset \widehat{\omega} \Subset \omega$. Note in particular that $\widetilde{\omega}_{T} \Subset \widehat{\omega}_{T} \Subset \omega_{T}$.

Next, because $\mathbf{v}$ is divergence free we also have, for all $t \in(0, T)$,

$$
-\Delta \mathbf{v}(t)=\operatorname{curl} w(t) \quad \text { in } \mathcal{O}, \quad \mathbf{v}(t)=0 \quad \text { on } \partial \mathcal{O} .
$$

Thus, using elliptic Carleman estimates with source term in $H^{-1}(\mathcal{O})$ with weight $e^{-s \varphi(t, \cdot)}$ and integrating in time, see [23], we immediately get

$$
s^{-1} \iint_{\mathcal{O}_{T}} \xi^{2}|\nabla \mathbf{v}|^{2} e^{-2 s \varphi}+s \lambda^{2} \iint_{\mathcal{O}_{T}} \xi^{4}|\mathbf{v}|^{2} e^{-2 s \varphi} \leq C\left(\iint_{\mathcal{O}_{T}} \xi^{3}|w|^{2} e^{-2 s \varphi}+s \lambda^{2} \iint_{\hat{\omega}_{T}} \xi^{4}|\mathbf{v}|^{2} e^{-2 s \varphi}\right) .
$$

Combined with (2.34), and using the fact that $w=\operatorname{curl} \mathbf{v}$ is bounded by $\partial_{\mathbf{n}} \mathbf{v}$ on $\Gamma_{T}$ (recall that $\mathbf{v}=0$ on $\Gamma_{T}$ ) and that $\xi^{*}=\xi$ and $\varphi^{*}=\varphi$ on $(0, T) \times \partial \mathcal{O}$, we immediately have that for some $s_{0}>1$ and $\lambda_{0}>1$, for all $s \geq s_{0}$ and $\lambda \geq \lambda_{0}$,

$$
\begin{aligned}
& s^{-1} \iint_{\mathcal{O}_{T}} \xi^{2}|\nabla \mathbf{v}|^{2} e^{-2 s \varphi}+\iint_{\mathcal{O}_{T}} \xi^{3}|w|^{2} e^{-2 s \varphi}+s \lambda^{2} \iint_{\mathcal{O}_{T}} \xi^{4}|\mathbf{v}|^{2} e^{-2 s \varphi} \\
& \quad \leq C\left(\iint_{\bar{\omega}_{T}} \xi^{3}|w|^{2} e^{-2 s \varphi}+s \lambda^{2} \iint_{\bar{\omega}_{T}} \xi^{4}|\mathbf{v}|^{2} e^{-2 s \varphi}+\lambda^{-1} \int_{\Gamma_{T}}\left(\xi^{*}\right)^{3}\left|\partial_{\mathbf{n}} \mathbf{v}\right|^{2} e^{-2 s \varphi^{*}}+s^{-1} \lambda^{-2} \iint_{\mathcal{O}_{T}} \xi^{2}|\mathbf{g}|^{2} e^{-2 s \varphi}\right) .
\end{aligned}
$$

We then introduce the stream function $\zeta$ associated to $\mathbf{v}$, i.e. $\mathbf{v}=\nabla^{\perp} \zeta$, which can be computed explicitly as the solution of (2.19) for some constants $c_{i}(t)$ due to the dimension $d=2$, see e.g. [19, Corollary 3.1]. Note that, by adding a constant to $\zeta$ if necessary, without loss of generality we can assume that (2.20) is also satisfied. Applying the elliptic Carleman estimate to Eq. (2.19) (see e.g. [17]), we obtain that

$$
s^{3} \lambda^{4} \iint_{\mathcal{O}_{T}} \xi^{6}|\zeta|^{2} e^{-2 s \varphi}+s \lambda^{2} \iint_{\mathcal{O}_{T}} \xi^{4}|\nabla \zeta|^{2} e^{-2 s \varphi} \leq C\left(\iint_{\mathcal{O}_{T}} \xi^{3}|w|^{2} e^{-2 s \varphi}+s^{3} \lambda^{4} \iint_{\bar{\omega}_{T}} \xi^{6}|\zeta|^{2} e^{-2 s \varphi}\right) .
$$


Note that the Carleman estimate of [17] is obtained for homogeneous Dirichlet boundary conditions. But it is easily seen that it remains true for a boundary data whose tangential derivative at the boundary vanishes, which is the case for $\zeta$.

Of course, estimate (2.34) requires an observation term in $\zeta$ in $\widehat{\omega_{T}}$. But Poincaré Wirtinger inequality (recall here that $\widehat{\omega}$ is assumed to be a domain, i.e. a connected open set) and condition (2.20) implies, for all $t \in[0, T]$,

$$
\int_{\widehat{\omega}}|\zeta(t, \cdot)|^{2} \leq C \int_{\widehat{\omega}}|\nabla \zeta(t, \cdot)|^{2}=\int_{\widehat{\omega}}|\operatorname{curl} \zeta(t, \cdot)|^{2}=\int_{\widehat{\omega}}|\mathbf{v}(t, \cdot)|^{2},
$$

and in particular:

$$
\iint_{\widehat{\omega_{T}}} \xi^{6}|\zeta|^{2} e^{-2 s \varphi} \leq C \iint_{\widehat{\omega_{T}}} \widehat{\xi}^{6}|\mathbf{v}|^{2} e^{-2 s \widehat{\varphi}}
$$

Let us stress the fact that the 2-d assumption is also used at this stage since (2.39) relies on the identity $|\nabla \zeta(t, \cdot)|^{2}=$ $|\operatorname{curl} \zeta(t, \cdot)|^{2}$.

Next, we use (2.38) to derive suitable weighted energy estimates for $\mathbf{v}$, hence for $\partial_{\mathbf{n}} \mathbf{v}$ on the boundary $\partial \mathcal{O}$. But since we do not have any estimate on the pressure in the Stokes equation (2.17), we are reduced to derive energy estimates for $\mathbf{v}$ with weight functions independent of $x$.

Estimates in $L^{2}\left(0, T ; \mathbf{H}^{1}(\mathcal{O})\right)$. We set $\left(\mathbf{v}_{a}, p_{a}\right): \stackrel{\text { def }}{=} \theta_{1}(t)(\mathbf{v}, p)$ with

$$
\theta_{1}(t): \stackrel{\text { def }}{=} s^{1 / 4} \lambda^{-1 / 4}\left(\xi^{*}\right)^{2-1 / m} e^{-s \varphi^{*}(t)} .
$$

Using

$$
\partial_{t} \varphi^{*} \leq C \lambda\left(\xi^{*}\right)^{1+1 / m} \quad \text { in } \mathcal{O}_{T}
$$

and explicit computations, we get

$$
\theta_{1}^{\prime} \geq-C s^{5 / 4} \lambda^{3 / 4}\left(\xi^{*}\right)^{3} e^{-s \varphi^{*}(t)} .
$$

The pair $\left(\mathbf{v}_{a}, p_{a}\right)$ satisfies

$$
\begin{cases}-\bar{\sigma} \partial_{t} \mathbf{v}_{a}-v \Delta \mathbf{v}_{a}+\nabla p_{a}=\theta_{1} \mathbf{g}-\bar{\sigma} \theta_{1}^{\prime} \mathbf{v} & \text { in } \mathcal{O}_{T}, \\ \operatorname{div} \mathbf{v}_{a}=0 & \text { in } \mathcal{O}_{T}, \\ \mathbf{v}_{a}=0 & \text { on } \Gamma_{T}, \\ \mathbf{v}_{a}(T)=0 & \text { in } \mathcal{O} .\end{cases}
$$

We want to obtain an estimate of the $L^{2}\left(\mathbf{H}_{0}^{1}\right)$-norm of $\mathbf{v}_{a}$, so we multiply the partial differential equation in (2.42) by $\mathbf{v}_{a}$, we integrate in $\mathcal{O}_{T}$ and we integrate by parts. This yields:

$$
\frac{1}{2}\left\|\sqrt{\bar{\sigma}(0, \cdot)} \mathbf{v}_{a}(0, \cdot)\right\|_{\mathbf{L}^{2}(\mathcal{O})}^{2}+v\left\|\mathbf{v}_{a}\right\|_{L^{2}\left(0, T ; \mathbf{H}_{0}^{1}(\mathcal{O})\right)}^{2}=\iint_{\mathcal{O}_{T}} \theta_{1} \mathbf{g} \cdot \mathbf{v}_{a}-\iint_{\mathcal{O}_{T}} \bar{\sigma} \theta_{1}^{\prime} \mathbf{v} \cdot \mathbf{v}_{a}-\frac{1}{2} \iint_{\mathcal{O}_{T}} \partial_{t} \bar{\sigma}\left|\mathbf{v}_{a}\right|^{2}
$$

First, we remark that

$$
\left|\iint_{\mathcal{O}_{T}} \theta_{1} \mathbf{g} \cdot \mathbf{v}_{a}\right| \leq \frac{v}{4} \iint_{\mathcal{O}_{T}}\left|\nabla \mathbf{v}_{a}\right|^{2}+C \int_{0}^{T}\left|\theta_{1}\right|^{2}\|\mathbf{g}\|_{\mathbf{H}^{-1}(\mathcal{O})}^{2} .
$$

We then focus on the second term of (2.43) and use (2.41)

$$
\begin{aligned}
& -\iint_{\mathcal{O}_{T}} \bar{\sigma} \theta_{1}^{\prime} \mathbf{v} \cdot \mathbf{v}_{a} \\
& \leq C s^{3 / 2} \lambda^{1 / 2} \iint_{\mathcal{O}_{T}}\left(\xi^{*}\right)^{5-1 / m} \mathbf{v} \cdot \nabla^{\perp} \zeta e^{-2 s \varphi^{*}(t)}
\end{aligned}
$$




$$
\begin{aligned}
& =-C s^{3 / 2} \lambda^{1 / 2} \iint_{\mathcal{O}_{T}}\left(\xi^{*}\right)^{5-1 / m} \operatorname{curl} \mathbf{v} \zeta e^{-2 s \varphi^{*}(t)} \\
& \leq C s^{5 / 2} \lambda^{3 / 2} \iint_{\mathcal{O}_{T}}\left(\xi^{*}\right)^{6}|\zeta|^{2} e^{-2 s \varphi^{*}(t)}+\frac{\nu s^{1 / 2} \lambda^{-1 / 2}}{4} \iint_{\mathcal{O}_{T}}\left(\xi^{*}\right)^{4-2 / m}|\nabla \mathbf{v}|^{2} e^{-2 s \varphi^{*}(t)} \\
& \leq C s^{5 / 2} \lambda^{3 / 2} \iint_{\mathcal{O}_{T}}\left(\xi^{*}\right)^{6}|\zeta|^{2} e^{-2 s \varphi^{*}(t)}+\frac{\nu}{4} \iint_{\mathcal{O}_{T}}\left|\nabla \mathbf{v}_{a}\right|^{2}
\end{aligned}
$$

The last term can be handled similarly:

$$
\begin{aligned}
\left.\left|\frac{1}{2} \iint_{\mathcal{O}_{T}} \partial_{t} \bar{\sigma}\right| \mathbf{v}_{a}\right|^{2} \mid & \leq C s^{1 / 2} \lambda^{-1 / 2} \iint_{\mathcal{O}_{T}}\left(\xi^{*}\right)^{4-2 / m}|\mathbf{v}|^{2} e^{-2 s \varphi^{*}} \\
& \leq C s^{5 / 2} \lambda^{3 / 2} \iint_{\mathcal{O}_{T}}\left(\xi^{*}\right)^{6}|\zeta|^{2} e^{-2 s \varphi^{*}(t)}+\frac{v}{4} \iint_{\mathcal{O}_{T}}\left|\nabla \mathbf{v}_{a}\right|^{2} .
\end{aligned}
$$

Plugging these three last estimates in (2.43), we obtain

$$
\left\|\mathbf{v}_{a}(0, \cdot)\right\|_{\mathbf{L}^{2}(\mathcal{O})}^{2}+\left\|\mathbf{v}_{a}\right\|_{L^{2}\left(0, T ; \mathbf{H}_{0}^{1}(\mathcal{O})\right)}^{2} \leq C\left(s^{5 / 2} \lambda^{3 / 2} \iint_{\mathcal{O}_{T}}\left(\xi^{*}\right)^{6}|\zeta|^{2} e^{-2 s \varphi^{*}}+\left\|\theta_{1} \mathbf{g}\right\|_{L^{2}\left(0, T ; \mathbf{H}^{-1}(\mathcal{O})\right)}^{2}\right) .
$$

Estimate in $L^{2}\left(0, T ; \mathbf{H}^{2}(\mathcal{O})\right)$. Let us now set $\left(\mathbf{v}_{b}, p_{b}\right): \stackrel{\text { def }}{=} \theta_{2}(t)(\mathbf{v}, p)$ with

$$
\theta_{2}(t): \stackrel{\text { def }}{=} s^{-1 / 4} \lambda^{-3 / 4}\left(\xi^{*}\right)^{3 / 2-3 /(2 m)} e^{-s \varphi^{*}(t)},
$$

for which explicit computations yield:

$$
\theta_{2}^{\prime} \geq-C s^{3 / 4} \lambda^{1 / 4}\left(\xi^{*}\right)^{\frac{5}{2}-\frac{1}{2 m}} e^{-s \varphi^{*}}
$$

This pair $\left(\mathbf{v}_{b}, p_{b}\right)$ satisfies

$$
\begin{cases}-\bar{\sigma} \partial_{t} \mathbf{v}_{b}-\Delta \mathbf{v}_{b}+\nabla p_{b}=\theta_{2} \mathbf{g}-\bar{\sigma} \theta_{2}^{\prime} \mathbf{v} & \text { in } \mathcal{O}_{T}, \\ \operatorname{div} \mathbf{v}_{b}=0 & \text { in } \mathcal{O}_{T}, \\ \mathbf{v}_{b}=0 & \text { on } \Gamma_{T}, \\ \mathbf{v}_{b}(T)=0 & \text { in } \mathcal{O} .\end{cases}
$$

We then multiply the partial differential equation in (2.47) by $\left(-\Delta \mathbf{v}_{b}+\nabla p_{b}\right) / \bar{\sigma}$, we integrate in $\mathcal{O}_{T}$ and we integrate by parts:

$$
\frac{1}{2} \int_{\mathcal{O}}\left|\nabla \mathbf{v}_{b}(0, \cdot)\right|^{2}+\iint_{\mathcal{O}_{T}} \frac{1}{\bar{\sigma}}\left|-\Delta \mathbf{v}_{b}+\nabla p_{b}\right|^{2}=\iint_{\mathcal{O}_{T}} \frac{\theta_{2}}{\bar{\sigma}} \mathbf{g}\left(-\Delta \mathbf{v}_{b}+\nabla p_{b}\right)-\iint_{\mathcal{O}_{T}} \theta_{2} \theta_{2}^{\prime}|\nabla \mathbf{v}|^{2} .
$$

Using (2.2) we can estimate the first term as follows:

$$
\left|\iint_{\mathcal{O}_{T}} \frac{\theta_{2}}{\bar{\sigma}} \mathbf{g}\left(-\Delta \mathbf{v}_{b}+\nabla p_{b}\right)\right| \leq \frac{1}{4} \iint_{\mathcal{O}_{T}} \frac{1}{\bar{\sigma}}\left|-\Delta \mathbf{v}_{b}+\nabla p_{b}\right|^{2}+C\left\|\theta_{2} \mathbf{g}\right\|_{\mathbf{L}^{2}\left(\mathcal{O}_{T}\right)}^{2} .
$$

For the second term, remark that by (2.46), we have

$$
\theta_{2} \theta_{2}^{\prime} \geq-C s^{1 / 2} \lambda^{-1 / 2}\left(\xi^{*}\right)^{4-2 / m} e^{-2 s \varphi^{*}}=-C \theta_{1}^{2},
$$

thus yielding

$$
-\iint_{\mathcal{O}_{T}} \theta_{2} \theta_{2}^{\prime}|\nabla \mathbf{v}|^{2} \leq C\left\|\theta_{1} \mathbf{v}\right\|_{L^{2}\left(0, T ; \mathbf{H}^{1}(\mathcal{O})\right)}^{2}=C\left\|\mathbf{v}_{a}\right\|_{L^{2}\left(0, T ; \mathbf{H}^{1}(\mathcal{O})\right)}^{2} .
$$


Therefore, using the above estimate and (2.49) into (2.48), we obtain

$$
\left\|\mathbf{v}_{b}\right\|_{L^{2}\left(0, T ; \mathbf{H}^{2}(\mathcal{O})\right)}^{2} \leq C \iint_{\mathcal{O}_{T}}\left|-\Delta \mathbf{v}_{b}+\nabla p_{b}\right|^{2} \leq C\left(\left\|\theta_{2} \mathbf{g}\right\|_{\mathbf{L}^{2}\left(\mathcal{O}_{T}\right)}^{2}+\left\|\mathbf{v}_{a}\right\|_{L^{2}\left(0, T ; \mathbf{H}^{1}(\mathcal{O})\right)}^{2}\right),
$$

where we have used the classical $H^{2}$-estimate for the stationary Stokes system, see e.g. [4, Theorem IV.5.8].

Global estimate on $\mathbf{v}$ and its normal derivative. Since $\mathbf{v}=0$ on $\Gamma_{T}$, classical estimates yield

$$
\left\|\partial_{\mathbf{n}} \mathbf{v}(t, \cdot)\right\|_{\mathbf{L}^{2}(\partial \mathcal{O})}^{2} \leq C\left(\|\mathbf{v}(t, \cdot)\|_{\mathbf{H}_{0}^{1}(\mathcal{O})}\|\mathbf{v}(t, \cdot)\|_{\mathbf{H}^{2}(\mathcal{O})}+\|\mathbf{v}(t, \cdot)\|_{\mathbf{H}_{0}^{1}(\mathcal{O})}^{2}\right),
$$

and in particular, using the fact that $\theta_{2}(t) \leq \theta_{1}(t)$ for all $t \in(0, T)$,

$$
\begin{aligned}
& \left\|\lambda^{-1 / 2}\left(\xi^{*}\right)^{\frac{7}{4}-\frac{5}{4 m}} \partial_{\mathbf{n}} \mathbf{v} e^{-s \varphi^{*}}(t, \cdot)\right\|_{\mathbf{L}^{2}(\partial \mathcal{O})}^{2} \\
& \quad \leq C\left(\left\|\theta_{1} \mathbf{v}(t, \cdot)\right\|_{\mathbf{H}_{0}^{1}(\mathcal{O})}\left\|\theta_{2} \mathbf{v}(t, \cdot)\right\|_{\mathbf{H}^{2}(\mathcal{O})}+\left\|\theta_{1} \mathbf{v}(t, \cdot)\right\|_{\mathbf{H}_{0}^{1}(\mathcal{O})}^{2}\right) .
\end{aligned}
$$

Putting together (2.45) and (2.50) with this last estimate, using (2.38) and (2.39) to estimate the term in $\zeta$ and taking into account that $m \geq 5$, we deduce that

$$
\begin{aligned}
& \left\|\mathbf{v}_{a}(0, \cdot)\right\|_{\mathbf{L}^{2}(\mathcal{O})}^{2}+\left\|\theta_{1} \mathbf{v}\right\|_{L^{2}\left(0, T ; \mathbf{H}_{0}^{1}(\mathcal{O})\right)}^{2}+\left\|\theta_{2} \mathbf{v}\right\|_{L^{2}\left(0, T ; \mathbf{H}^{2}(\mathcal{O})\right)}^{2}+\lambda^{-1}\left\|\left(\xi^{*}\right)^{3 / 2} \partial_{\mathbf{n}} \mathbf{v} e^{-s \varphi^{*}}\right\|_{\mathbf{L}^{2}\left(\Gamma_{T}\right)}^{2} \\
& \quad \leq C\left(s^{-1 / 2} \lambda^{-5 / 2} \iint_{\mathcal{O}_{T}} \xi^{3}|w|^{2} e^{-2 s \varphi}+s^{5 / 2} \lambda^{3 / 2} \iint_{\widehat{\omega_{T}}} \widehat{\xi}^{6}|\mathbf{v}|^{2} e^{-2 s \widehat{\varphi}}+\left\|\theta_{1} \mathbf{g}\right\|_{L^{2}\left(0, T ; \mathbf{H}^{-1}(\mathcal{O})\right)}^{2}+\left\|\theta_{2} \mathbf{g}\right\|_{\mathbf{L}^{2}\left(\mathcal{O}_{T}\right)}^{2}\right) .
\end{aligned}
$$

Elimination of the boundary term. We come back to the Carleman inequality (2.37) and we combine it with (2.51): for $s$ large enough,

$$
\begin{aligned}
& \left\|\mathbf{v}_{a}(0, \cdot)\right\|_{\mathbf{L}^{2}(\mathcal{O})}^{2}+\left\|\theta_{1} \mathbf{v}\right\|_{L^{2}\left(0, T ; \mathbf{H}_{0}^{1}(\mathcal{O})\right)}^{2}+\left\|\theta_{2} \mathbf{v}\right\|_{L^{2}\left(0, T ; \mathbf{H}^{2}(\mathcal{O})\right)}^{2} \\
& \quad+s^{-1} \iint_{\mathcal{O}_{T}} \xi^{2}|\nabla \mathbf{v}|^{2} e^{-2 s \varphi}+\iint_{\mathcal{O}_{T}} \xi^{3}|w|^{2} e^{-2 s \varphi}+s \lambda^{2} \iint \xi^{4}|\mathbf{v}|^{2} e^{-2 s \varphi} \\
& \leq C\left(\iint_{\widehat{\omega_{T}}} \xi^{3}|w|^{2} e^{-2 s \varphi}+s^{5 / 2} \lambda^{2} \iint \widehat{\xi}^{6}|\mathbf{v}|^{2} e^{-2 s \widehat{\varphi}}\right. \\
& \left.\quad+\left\|\theta_{1} \mathbf{g}\right\|_{L^{2}\left(0, T ; \mathbf{H}^{-1}(\mathcal{O})\right)}^{2}+\left\|\theta_{2} \mathbf{g}\right\|_{\mathbf{L}^{2}\left(\mathcal{O}_{T}\right)}^{2}+s^{-1} \lambda^{-2} \iint_{\mathcal{O}_{T}} \xi^{2}|\mathbf{g}|^{2} e^{-2 s \varphi}\right) .
\end{aligned}
$$

Removing the observation on $w$. We now estimate the local term in $|w|^{2}$. For this purpose, we recall that $\widehat{\omega_{T}}=$ $[0, T] \times \widehat{\omega} \Subset \omega_{T}=[0, T] \times \omega$ and we consider a positive function $\chi \in C^{2}(\overline{\mathcal{O}})$ such that

$$
\chi=1 \quad \text { in } \widehat{\omega}, \quad \chi=0 \quad \text { in } \mathcal{O} \backslash \omega .
$$

Using

$$
\iint_{\widehat{\omega}_{T}} \xi^{3}|w|^{2} e^{-2 s \varphi} \leq \iint_{\widehat{\omega}_{T}} \widehat{\xi}^{3}|w|^{2} e^{-2 s \widehat{\varphi}},
$$

we are reduced to estimate the right hand side of (2.53):

$$
\begin{aligned}
\iint_{\widehat{\omega}_{T}} \widehat{\xi}^{3}|w|^{2} e^{-2 s \widehat{\varphi}} & \leq \iint_{\omega_{T}} \chi \widehat{\xi}^{3}|w|^{2} e^{-2 s \widehat{\varphi}} \leq \iint_{\omega_{T}} \chi \widehat{\xi}^{3}|\nabla \mathbf{v}|^{2} e^{-2 s \widehat{\varphi}} \\
& =-\iint_{\omega_{T}} \chi \widehat{\xi}^{3} \Delta \mathbf{v} \mathbf{v} e^{-2 s \widehat{\varphi}}+\frac{1}{2} \iint_{\omega_{T}} \Delta \chi \widehat{\xi}^{3}|\mathbf{v}|^{2} e^{-2 s \widehat{\varphi}}
\end{aligned}
$$




$$
\leq \varepsilon s^{-1 / 2} \lambda^{-3 / 2} \iint_{\mathcal{O}_{T}}\left(\xi^{*}\right)^{3-3 / m}|\Delta \mathbf{v}|^{2} e^{-2 s \varphi^{*}}+C_{\varepsilon} s^{1 / 2} \lambda^{3 / 2} \iint_{\omega_{T}}\left(\xi^{*}\right)^{-3+3 / m} \widehat{\xi}^{6}|\mathbf{v}|^{2} e^{2 s \varphi^{*}-4 s \widehat{\varphi}},
$$

where the last estimate follows from Young's identity and where $\varepsilon>0$.

Using the last above inequality in (2.52) with $\varepsilon$ small enough and recalling the definition of $\theta_{2}$, we get in particular

$$
\begin{aligned}
& s^{1 / 2} \lambda^{-1 / 2} \int_{\mathcal{O}}\left(\xi^{*}\right)^{4-2 / m}|\mathbf{v}(0, \cdot)|^{2} e^{-2 s \varphi^{*}}+s^{1 / 2} \lambda^{-1 / 2} \int_{0}^{T}\left(\xi^{*}\right)^{4-2 / m} e^{-2 s \varphi^{*}}\|\mathbf{v}\|_{\mathbf{H}^{1}(\mathcal{O})}^{2} \\
& \quad+s^{-1} \iint_{\mathcal{O}_{T}} \xi^{2}|\nabla \mathbf{v}|^{2} e^{-2 s \varphi}+s \lambda^{2} \iint_{\mathcal{O}_{T}} \xi^{4}|\mathbf{v}|^{2} e^{-2 s \varphi}+\iint_{\mathcal{O}_{T}} \xi^{3}|\operatorname{curl} \mathbf{v}|^{2} e^{-2 s \varphi} \\
& \leq C\left(s^{5 / 2} \lambda^{2} \iint_{\omega_{T}} \widehat{\xi}^{6}|\mathbf{v}|^{2} e^{2 s \varphi^{*}-4 s \widehat{\varphi}}+s^{-1} \lambda^{-2} \iint_{\mathcal{O}_{T}} \xi^{2}|\mathbf{g}|^{2} e^{-2 s \varphi}+s^{1 / 2} \lambda^{-1 / 2} \int_{0}^{T}\left(\xi^{*}\right)^{4-2 / m} e^{-2 s \varphi^{*}}\|\mathbf{g}\|_{\mathbf{H}^{-1}(\mathcal{O})}^{2}\right. \\
& \left.\quad+s^{-1 / 2} \lambda^{-3 / 2} \iint_{\mathcal{O}_{T}}\left(\xi^{*}\right)^{3-3 / m}|\mathbf{g}|^{2} e^{-2 s \varphi^{*}}\right) .
\end{aligned}
$$

This concludes the proof of Theorem 2.7.

\subsubsection{Proof of Theorem 2.1}

Let $\mathbf{v}$ be a smooth solution of (2.6) with source term $\mathbf{g}$. Then $\mathbf{v}$ is a solution of (2.17) with source term

$$
\tilde{\mathbf{g}}=\mathbf{g}+\partial_{t} \bar{\sigma} \mathbf{v}+D(\bar{\sigma} \mathbf{v}) \overline{\mathbf{y}}+\bar{\sigma} \mathbf{v} \operatorname{div}(\overline{\mathbf{y}}) .
$$

Applying Theorem 2.7 to $\mathbf{v}$ with source term $\tilde{\mathbf{g}}$, for all $s \geq s_{0}$ and $\lambda \geq \lambda_{0}$ we get

$$
\begin{aligned}
& s^{1 / 2} \lambda^{-1 / 2} \int_{\mathcal{O}}\left(\xi^{*}\right)^{4-2 / m}|\mathbf{v}(0, \cdot)|^{2} e^{-2 s \varphi^{*}(0)}+s \lambda^{2} \iint_{\mathcal{O}_{T}} \xi^{4}|\mathbf{v}|^{2} e^{-2 s \varphi} \\
& \quad+s^{1 / 2} \lambda^{-1 / 2} \int_{0}^{T}\left(\xi^{*}\right)^{4-2 / m} e^{-2 s \varphi^{*}}\|\mathbf{v}\|_{\mathbf{H}^{1}(\mathcal{O})}^{2}+\iint_{\mathcal{O}_{T}} \xi^{3}|\operatorname{curl} \mathbf{v}|^{2} e^{-2 s \varphi}+s^{-1} \iint_{\mathcal{O}_{T}} \xi^{2}|\nabla \mathbf{v}|^{2} e^{-2 s \varphi} \\
& \leq C\left(s^{5 / 2} \lambda^{2} \iint_{\omega_{T}} \widehat{\xi}^{6}|\mathbf{v}|^{2} e^{2 s \varphi^{*}-4 s \widehat{\varphi}}+s^{-1} \lambda^{-2} \iint_{\mathcal{O}_{T}} \xi^{2}|\tilde{\mathbf{g}}|^{2} e^{-2 s \varphi}\right. \\
& \left.\quad+s^{1 / 2} \lambda^{-1 / 2} \int_{0}^{T}\left(\xi^{*}\right)^{4-2 / m} e^{-2 s \varphi^{*}}\|\tilde{\mathbf{g}}\|_{\mathbf{H}^{-1}(\mathcal{O})}^{2}+s^{-1 / 2} \lambda^{-3 / 2} \iint_{\mathcal{O}_{T}}\left(\xi^{*}\right)^{3-3 / m}|\tilde{\mathbf{g}}|^{2} e^{-2 s \varphi^{*}}\right)
\end{aligned}
$$

and we are thus reduced to estimate the last terms of the inequality.

But we have

$$
\begin{aligned}
& s^{-1} \lambda^{-2} \iint_{\mathcal{O}_{T}} \xi^{2}|\tilde{\mathbf{g}}|^{2} e^{-2 s \varphi} \\
& \quad \leq C\left(s^{-1} \lambda^{-2} \iint_{\mathcal{O}_{T}} \xi^{2}|\mathbf{g}|^{2} e^{-2 s \varphi}+s^{-1} \lambda^{-2} \iint_{\mathcal{O}_{T}} \xi^{2}|\mathbf{v}|^{2} e^{-2 s \varphi}+s^{-1} \lambda^{-2} \iint_{\mathcal{O}_{T}} \xi^{2}|\nabla \mathbf{v}|^{2} e^{-2 s \varphi}\right), \\
& s^{-1 / 2} \lambda^{-3 / 2} \iint_{\mathcal{O}_{T}}\left(\xi^{*}\right)^{3-3 / m}|\tilde{\mathbf{g}}|^{2} e^{-2 s \varphi^{*}}
\end{aligned}
$$




$$
\begin{aligned}
\leq & C\left(s^{-1 / 2} \lambda^{-3 / 2} \iint_{\mathcal{O}_{T}}\left(\xi^{*}\right)^{3-3 / m}|\mathbf{g}|^{2} e^{-2 s \varphi^{*}}\right. \\
& \left.+s^{-1 / 2} \lambda^{-3 / 2} \iint_{\mathcal{O}_{T}}\left(\xi^{*}\right)^{3-3 / m}|\mathbf{v}|^{2} e^{-2 s \varphi^{*}}+s^{-1 / 2} \lambda^{-3 / 2} \iint_{\mathcal{O}_{T}}\left(\xi^{*}\right)^{3-3 / m}|\nabla \mathbf{v}|^{2} e^{-2 s \varphi^{*}}\right),
\end{aligned}
$$

in which all the terms in $\mathbf{v}, \nabla \mathbf{v}$ can be absorbed by the left-hand side of (2.55) for $s$ and $\lambda$ large enough.

We also have, for all $t \in(0, T)$,

$$
\|\tilde{\mathbf{g}}(t)\|_{\mathbf{H}^{-1}(\mathcal{O})}^{2} \leq C\|\mathbf{g}(t, \cdot)\|_{\mathbf{L}^{2}(\mathcal{O})}^{2}+C\|\mathbf{v}(t, \cdot)\|_{\mathbf{L}^{2}(\mathcal{O})}^{2} .
$$

Hence

$$
\begin{aligned}
s^{1 / 2} \lambda^{-1 / 2} \int_{0}^{T}\left(\xi^{*}\right)^{4-2 / m} e^{-2 s \varphi^{*}}\|\tilde{\mathbf{g}}\|_{\mathbf{H}^{-1}(\mathcal{O})}^{2} \leq & C s^{1 / 2} \lambda^{-1 / 2} \iint_{\mathcal{O}_{T}}\left(\xi^{*}\right)^{4-2 / m} e^{-2 s \varphi^{*}}|\mathbf{g}|^{2} \\
& +C s^{1 / 2} \lambda^{-1 / 2} \iint_{\mathcal{O}_{T}}\left(\xi^{*}\right)^{4-2 / m} e^{-2 s \varphi^{*}}|\mathbf{v}|^{2}
\end{aligned}
$$

Plugging these last estimates in (2.55), we obtain (2.16) for $s$ and $\lambda$ large enough.

\subsection{Proof of Theorem 2.3}

We use the following simplified form of (2.16): for all $s \geq s_{0}$ and $\lambda \geq \lambda_{0}$ and all smooth solutions $\mathbf{v}$ of (2.6) with source term $\mathbf{g}$ :

$$
\begin{aligned}
& \int_{\mathcal{O}}\left(\xi^{*}\right)^{4-2 / m}|\mathbf{v}(0, \cdot)|^{2} e^{-2 s \varphi^{*}(0)}+s^{1 / 2} \lambda^{5 / 2} \iint_{\mathcal{O}_{T}} \xi^{4}|\mathbf{v}|^{2} e^{-2 s \varphi} \\
& \quad \leq C\left(s^{2} \lambda^{5 / 2} \iint_{\omega_{T}} \widehat{\xi}^{6}|\mathbf{v}|^{2} e^{2 s \varphi^{*}-4 s \widehat{\varphi}}+\iint_{\mathcal{O}_{T}} \xi^{4-2 / m}|\mathbf{g}|^{2} e^{-2 s \varphi}\right) .
\end{aligned}
$$

Easy density arguments then show that this result extends to all solutions $\mathbf{v}$ of (2.6) with source term $\mathbf{g} \in L^{2}\left(\mathcal{O}_{T}\right)$ and final data $\mathbf{v}(T)=\mathbf{v}_{T} \in \mathbf{V}_{0}^{1}(\Omega)$.

We then follow the proof of Theorem 2.6 and introduce the functional $J_{S t}$ defined by

$$
\begin{aligned}
J_{S t}\left(\mathbf{v}_{T}, \mathbf{g}\right): \stackrel{\text { def }}{=} & \frac{1}{2} \iint_{\mathcal{O}_{T}} \xi^{4-2 / m}|\mathbf{g}|^{2} e^{-2 s \varphi}+\frac{s^{2} \lambda^{5 / 2}}{2} \iint_{\omega_{T}} \widehat{\xi}^{6}|\mathbf{v}|^{2} e^{2 s \varphi^{*}-4 s \widehat{\varphi}} \\
& -\iint_{\mathcal{O}_{T}} \mathbf{f} \cdot \mathbf{v}-\int_{\mathcal{O}} \mathbf{u}_{0}(\cdot) \cdot \mathbf{v}(0, \cdot),
\end{aligned}
$$

defined for data $\left(\mathbf{v}_{T}, \mathbf{g}\right) \in \mathbf{V}_{0}^{1}(\Omega) \times L^{2}\left(\mathcal{O}_{T}\right)$, where $\mathbf{v}$ solves (2.6) with $\mathbf{v}(T)=\mathbf{v}_{T}$.

We then need to define the functional $J_{S t}$ on the set $\mathbf{X}_{S t, o b s}: \stackrel{\text { def }}{=} \overline{\mathbf{X}_{S t, o b s}^{0}}\|\cdot\|_{t, o b s}$, where

$$
\mathbf{X}_{S t, o b s}^{0}: \stackrel{\text { def }}{=}\left\{\left(\mathbf{v}_{T}, \mathbf{g}\right) \in \mathbf{V}_{0}^{1}(\Omega) \times L^{2}\left(\mathcal{O}_{T}\right)\right\}
$$

and the norm $\left\|\left(\mathbf{v}_{T}, \mathbf{g}\right)\right\|_{S t, o b s}$ is defined by

$$
\left\|\left(\mathbf{v}_{T}, \mathbf{g}\right)\right\|_{S t, o b s}^{2}: \stackrel{\text { def }}{=} \iint_{\mathcal{O}_{T}} \xi^{4-2 / m}|\mathbf{g}|^{2} e^{-2 s \varphi}+s^{2} \lambda^{5 / 2} \iint_{\omega_{T}} \widehat{\xi}^{6}|\mathbf{v}|^{2} e^{2 s \varphi^{*}-4 s \widehat{\varphi}},
$$

where $\mathbf{v}$ is the corresponding solution to (2.6). 
According to (2.57), the functional $J_{S t}$ can be extended by continuity on $\mathbf{X}_{S t, o b s}$ if $\mathbf{f}$ satisfies (2.21). The functional $J_{S t}$ then has a unique minimizer on $\mathbf{X}_{S t, o b s}$, that we denote $\left(\mathbf{V}_{T}, \mathbf{G}\right)$ and which corresponds to a solution $\mathbf{V}$ of (2.6). We get, for all smooth solution $\mathbf{v}$ of (2.6) corresponding to a source term $\mathbf{g}$,

$$
0=\iint_{\mathcal{O}_{T}} \xi^{4-2 / m} \mathbf{G} \cdot \mathbf{g} e^{-2 s \varphi}+s^{2} \lambda^{5 / 2} \iint_{\omega_{T}} \widehat{\xi}^{6} \mathbf{V} \cdot \mathbf{v} e^{2 s \varphi^{*}-4 s \widehat{\varphi}}-\iint_{\mathcal{O}_{T}} \mathbf{f} \cdot \mathbf{v}-\int_{\mathcal{O}} \mathbf{u}_{0}(\cdot) \cdot \mathbf{v}(0, \cdot) .
$$

In particular, setting

$$
\mathbf{u}=\xi^{4-2 / m} \mathbf{G} e^{-2 s \varphi}, \quad \mathbf{h}=-s^{2} \lambda^{5 / 2 \widehat{\xi}^{6}} \mathbf{V} e^{2 s \varphi^{*}-4 s \widehat{\varphi}} 1_{\omega_{T}},
$$

we obtain a solution in the sense of transposition of the control problem (2.4)-(2.5) with a control term acting only on $\omega_{T}$.

Besides, using again the Carleman estimate (2.57) and the fact that $J_{S t}\left(\mathbf{V}_{T}, \mathbf{G}\right) \leq J_{S t}(0,0)=0$, one immediately derives that

$$
\left\|\left(\mathbf{V}_{T}, \mathbf{G}\right)\right\|_{o b s}^{2} \leq \frac{C}{s^{1 / 2} \lambda^{5 / 2}} \iint_{\mathcal{O}_{T}} \xi^{-4}|\mathbf{f}|^{2} e^{2 s \varphi}+C \int_{\mathcal{O}}\left(\xi^{*}\right)^{2 / m-4}\left|\mathbf{u}_{0}\right|^{2} e^{2 s \varphi^{*}(0)} .
$$

Hence, using (2.61), the controlled trajectory $(\mathbf{u}, \mathbf{h})$ satisfies

$$
\iint_{\mathcal{O}_{T}} \xi^{2 / m-4}|\mathbf{u}|^{2} e^{2 s \varphi}+\frac{1}{s^{2} \lambda^{5 / 2}} \iint_{\omega_{T}} \widehat{\xi}^{-6}|\mathbf{h}|^{2} e^{4 s \widehat{\varphi}-2 s \varphi^{*}} \leq \frac{C}{s^{1 / 2} \lambda^{5 / 2}} \iint_{\mathcal{O}_{T}} \xi^{-4}|\mathbf{f}|^{2} e^{2 s \varphi}+\int_{\mathcal{O}}\left(\xi^{*}\right)^{2 / m-4}\left|\mathbf{u}_{0}\right|^{2} e^{2 s \varphi^{*}(0)} .
$$

Finally, we can then derive $H^{1}\left(\mathbf{L}^{2}\right) \cap L^{2}\left(\mathbf{H}^{2}\right)$ estimates on $\mathbf{u}$ by applying regularity results for Stokes equations to the system satisfied by $e^{\frac{3}{4} s \varphi^{*}} \mathbf{u}$. The computations are left to the reader.

\section{Controlling the density}

This section is devoted to explain how to solve the control problem (1.19). As we said in the introduction, the main difficulty is that we need to provide a controlled trajectory that can be estimated with the use of the weight functions introduced in Section 2.

\subsection{Basic properties of the flow}

Let $\overline{\mathbf{y}}$ be an extension of $\overline{\mathbf{y}}$ on $[0, T] \times \mathbb{R}^{2}$ and $\bar{X}$ the corresponding flow, defined in (1.6). As $\overline{\mathbf{y}} \in \mathbf{C}^{2}\left([0, T] \times \mathbb{R}^{2}\right)$, the flow $\bar{X}$ is continuous with respect to the variables $(t, \tau, x) \in[0, T]^{2} \times \mathbb{R}^{2}$.

We first discuss the stability of property (1.8):

Lemma 3.1. Assume that $\overline{\mathbf{y}} \in \mathbf{C}^{2}\left([0, T] \times \mathbb{R}^{2}\right)$, and that the flow $\bar{X}$ defined by (1.6) satisfies (1.8).

There exist $\varepsilon>0, T_{0}^{*}>0$ and $T_{1}^{*}>0$ such that for all $T_{0} \in\left(0, T_{0}^{*}\right)$, for all $T_{1} \in\left(0, T_{1}^{*}\right)$ and for all $x \in \bar{\Omega}$, there exists $t \in\left[T_{0}, T-2 T_{1}\right]$ such that $d\left(\bar{X}\left(t, T_{0}, x\right), \Omega\right) \geq 2 \varepsilon$.

Proof. The proof is done by contradiction. Assume it is false. Then for all $\varepsilon>0$, there exist $T_{0}^{\varepsilon}>0$ and $T_{1}^{\varepsilon}$ such that $T_{0}^{\varepsilon}, T_{1}^{\varepsilon}$ converge to 0 as $\varepsilon \rightarrow 0$, and an $x_{\varepsilon}$ in $\bar{\Omega}$ such that

$$
\forall t \in\left[T_{0}^{\varepsilon}, T-2 T_{1}^{\varepsilon}\right], \quad d\left(\bar{X}\left(t, T_{0}^{\varepsilon}, x_{\varepsilon}\right), \Omega\right)<2 \varepsilon .
$$

But $x_{\varepsilon}$ is bounded in $\bar{\Omega}$. Hence, up to a subsequence, it converges to some $\bar{x}$ in $\bar{\Omega}$. As the flow $\bar{X}$ is continuous in $[0, T]^{2} \times \mathbb{R}^{2}$ and the distance function is continuous, for each $t \in(0, T)$, one could then pass to the limit in (3.1):

$$
\forall t \in(0, T), \quad d(\bar{X}(t, 0, \bar{x}), \Omega)=0 .
$$

This is of course in contradiction with (1.8). 
For $\widehat{\mathbf{u}} \in L^{2}\left(0, T ; \mathbf{H}^{2}\left(\mathbb{R}^{2}\right)\right)$ we denote by $\widehat{X}$ the flow defined by

$$
\partial_{t} \widehat{X}(t, \tau, x)=(\overline{\mathbf{y}}+\widehat{\mathbf{u}})(t, \widehat{X}(t, \tau, x)), \quad \widehat{X}(\tau, \tau, x)=x .
$$

Note that in dimension 2, the flow $\widehat{X}$ associated to a velocity field in $L^{2}\left(0, T ; \mathbf{H}^{2}\left(\mathbb{R}^{2}\right)\right)$ is well-defined in the classical sense thanks to Osgood's condition, see [28].

We then show that, provided $\widehat{\mathbf{u}}$ is small enough, the property (1.8) also holds for $\widehat{X}$ :

Lemma 3.2. Under the setting of Lemma 3.1, there exists $\varsigma>0$ such that for all $\widehat{\mathbf{u}} \in L^{2}\left(0, T ; \mathbf{H}^{2}\left(\mathbb{R}^{2}\right)\right)$, satisfying

$$
\|\widehat{\mathbf{u}}\|_{L^{2}\left(0, T ; \mathbf{L}^{\infty}\left(\mathbb{R}^{2}\right)\right)} \leq 2 \varsigma,
$$

the flow $\widehat{X}$ defined by (3.2) satisfies the following property: for all $T_{0} \in\left(0, T_{0}^{*}\right)$, for all $T_{1} \in\left(0, T_{1}^{*}\right)$ and for all $x \in \bar{\Omega}$, there exists $t \in\left[T_{0}, T-2 T_{1}\right]$ such that $d\left(\widehat{X}\left(t, T_{0}, x\right), \Omega\right) \geq \varepsilon$.

Proof. Set $L=\|\nabla \overline{\mathbf{y}}\|_{L^{\infty}\left(0, T ; \mathbf{L}^{\infty}(\Omega)\right)}$. For $\tau, t \in[0, T]^{2}$ with $t \geq \tau$ and $x \in \mathbb{R}^{2}$, we have:

$$
\begin{aligned}
|\widehat{X}(t, \tau, x)-\bar{X}(t, \tau, x)| & =|\widehat{X}(t, \tau, x)-\widehat{X}(\tau, \tau, x)+\bar{X}(\tau, \tau, x)-\bar{X}(t, \tau, x)| \\
& =\left|\int_{\tau}^{t}\left(\partial_{t} \widehat{X}\left(t^{\prime}, \tau, x\right)-\partial_{t} \bar{X}\left(t^{\prime}, \tau, x\right)\right) \mathrm{d} t^{\prime}\right| \\
& =\left|\int_{\tau}^{t} \widehat{\mathbf{u}}\left(t^{\prime}, \widehat{X}\left(t^{\prime}, \tau, x\right)\right)+\overline{\mathbf{y}}\left(t^{\prime}, \widehat{X}\left(t^{\prime}, \tau, x\right)\right)-\overline{\mathbf{y}}\left(t^{\prime}, \bar{X}\left(t^{\prime}, \tau, x\right)\right) \mathrm{d} t^{\prime}\right| \\
& \leq|t-\tau|^{1 / 2}\|\widehat{\mathbf{u}}\|_{L^{2}\left(\tau, t ; \mathbf{L}^{\infty}\left(\mathbb{R}^{2}\right)\right)}+L \int_{\tau}^{t}\left|\widehat{X}\left(t^{\prime}, \tau, x\right)-\bar{X}\left(t^{\prime}, \tau, x\right)\right| \mathrm{d} t^{\prime} .
\end{aligned}
$$

Then Gronwall's Lemma yields for all $t \in[0, T]$ and $x \in \mathbb{R}^{2}$ :

$$
|\widehat{X}(t, \tau, x)-\bar{X}(t, \tau, x)| \leq T^{1 / 2} e^{L T}\|\widehat{\mathbf{u}}\|_{L^{2}\left(\tau, t ; \mathbf{L}^{\infty}\left(\mathbb{R}^{2}\right)\right)} .
$$

According to Lemma 3.1, Lemma 3.2 thus holds by setting $\varsigma=T^{-1 / 2} e^{-L T} \varepsilon / 2$ in (3.3).

\subsection{Construction of the controlled density}

In this section, we assume that

$$
\widehat{\mathbf{u}} \in L^{2}\left(0, T ; \mathbf{H}^{2}\left(\mathbb{R}^{2}\right)\right) \text { and }\|\widehat{\mathbf{u}}\|_{L^{2}\left(0, T ; \mathbf{L}^{\infty}\left(\mathbb{R}^{2}\right)\right)} \leq 2 \varsigma,
$$

where $\varsigma$ is given by Lemma 3.2. We then choose $T_{0} \in\left(0, T_{0}^{*}\right)$ and $T_{1} \in\left(0, T_{1}^{*}\right)$, where $T_{0}^{*}, T_{1}^{*}$ are given by Lemma 3.2.

The construction of the controlled density $\rho$ solution of (1.19) is then done as in [11]: we construct a forward solution $\rho_{f}$ and a backward solution $\rho_{b}$ of the transport equation in (1.19) and we glue these two solutions according to the characteristics of the flow.

Indeed, we define $\rho_{f}$ as the solution of

$$
\begin{cases}\partial_{t} \rho_{f}+(\overline{\mathbf{y}}+\widehat{\mathbf{u}}) \cdot \nabla \rho_{f}=-\widehat{\mathbf{u}} \cdot \nabla \bar{\sigma} & \text { in } \Omega_{T}, \\ \rho_{f}(t, x)=0 & \text { for } t \in(0, T), x \in \partial \Omega, \text { with }(\overline{\mathbf{y}}(t, x)+\widehat{\mathbf{u}}(t, x)) \cdot \mathbf{n}(x)<0, \\ \rho_{f}(0)=\rho_{0} & \text { in } \Omega,\end{cases}
$$

and $\rho_{b}$ as the solution of

$$
\begin{cases}\partial_{t} \rho_{b}+(\overline{\mathbf{y}}+\widehat{\mathbf{u}}) \cdot \nabla \rho_{b}=-\widehat{\mathbf{u}} \cdot \nabla \bar{\sigma} & \text { in } \Omega_{T}, \\ \rho_{b}=0 & \text { for } t \in(0, T), x \in \partial \Omega, \text { with }(\overline{\mathbf{y}}(t, x)+\widehat{\mathbf{u}}(t, x)) \cdot \mathbf{n}(x)>0, \\ \rho_{b}(T)=0 & \text { in } \Omega .\end{cases}
$$


We also introduce $\chi$ the solution of

$$
\begin{cases}\partial_{t} \chi+(\overline{\mathbf{y}}+\widehat{\mathbf{u}}) \cdot \nabla \chi=0 & \text { in } \Omega_{T}, \\ \chi=1_{t \in\left(0, T_{0}\right)}(t) & \text { for } t \in(0, T), x \in \partial \Omega, \text { with }(\overline{\mathbf{y}}(t, x)+\widehat{\mathbf{u}}(t, x)) \cdot \mathbf{n}(x)<0, \\ \chi(0)=1 & \text { in } \Omega .\end{cases}
$$

We finally define $\rho(t, x)$ as follows,

$$
\rho(t, x): \stackrel{\text { def }}{=}(1-\chi(t, x)) \rho_{b}(t, x)+\chi(t, x) \rho_{f}(t, x) .
$$

It is easy to check that this function $\rho$ satisfies the transport equation $(1.19)_{(1)}$ and the required initial condition $(1.19)_{(2)}$. The final condition $\rho(T)=0$ in $(1.19)_{(3)}$ is satisfied due to the properties of the flow proved in Lemma 3.2, which guarantees that $\chi(T)=0$.

In the next subsections, we describe how to get estimates on the function $\rho$ constructed in (3.9) in the weighted spaces adapted to the Carleman estimates derived in Section 2.

\subsection{Explicit description of the density}

To begin with, let us remark that the function $\chi$ is explicitly given by:

$$
\chi(t, x)= \begin{cases}1 & \text { if } t<T_{0} \\ 1 & \text { if } t \geq T_{0} \text { and } X(\tau, t, x) \in \Omega \text { for all } \tau \in\left[T_{0}, t\right], \\ 0 & \text { else, }\end{cases}
$$

so that from Lemma 3.2 we have in particular

$$
\chi(t, x)=0 \quad \text { and } \quad \rho(t, x)=\rho_{b}(t, x) \quad \text { for }(t, x) \in\left[T-2 T_{1}, T\right] \times \Omega .
$$

We also give explicit expressions for $\rho_{f}$ and $\rho_{b}$. In order to do that, for $t \in[0, T]$, we introduce

$$
\begin{aligned}
& \Omega_{[0]}(t): \stackrel{\text { def }}{=}\{x \in \Omega \mid \widehat{X}(\tau, t, x) \in \Omega \text { for all } \tau \in[0, t]\} \\
& \Omega_{[T]}(t): \stackrel{\text { def }}{=}\{x \in \Omega \mid \widehat{X}(\tau, t, x) \in \Omega \text { for all } \tau \in[t, T]\}
\end{aligned}
$$

and for all $(t, x) \in[0, T] \times \Omega$ :

$$
\begin{aligned}
t_{\text {in }}(t, x) & : \stackrel{\text { def }}{=} \sup \{\tau \in[0, t) \mid \widehat{X}(\tau, t, x) \in \partial \Omega\}, \\
t_{\text {out }}(t, x) & : \stackrel{\text { def }}{=} \inf \{\tau \in(t, T] \mid \widehat{X}(\tau, t, x) \in \partial \Omega\} .
\end{aligned}
$$

In the above definitions, we use the convention $\sup \emptyset=0$ and $\inf \emptyset=T$. This way, $t_{\text {in }}(t, x)=0$ iff $x \in \Omega_{[0]}(t)$ and $t_{\text {out }}(t, x)=T$ iff $x \in \Omega_{[T]}(t)$.

Using these notations, $\rho_{f}$ and $\rho_{b}$ are explicitly given by

$$
\begin{aligned}
& \rho_{f}(t, x)= \begin{cases}\rho_{0}(\widehat{X}(0, t, x))-\int_{0}^{t}(\widehat{\mathbf{u}} \cdot \nabla \bar{\sigma})(\tau, \widehat{X}(\tau, t, x)) \mathrm{d} \tau & \text { if } x \in \Omega_{[0]}(t), \\
-\int_{t_{\mathrm{in}}(t, x)}^{t}(\widehat{\mathbf{u}} \cdot \nabla \bar{\sigma})(\tau, \widehat{X}(\tau, t, x)) \mathrm{d} \tau & \text { else, }\end{cases} \\
& \rho_{b}(t, x)=\int_{t_{\text {out }}(t, x)}^{t}(\widehat{\mathbf{u}} \cdot \nabla \bar{\sigma})(\tau, \widehat{X}(\tau, t, x)) \mathrm{d} \tau \quad \text { for } x \in \Omega .
\end{aligned}
$$

We are now in position to derive weighted estimates on $\rho$.

\subsection{Weighted estimates on the density}

In order to derive weighted estimates on $\rho$ based on the Carleman weights $\psi, \theta, \varphi, \xi$ described in (2.7)-(2.11), we will need some further assumptions. 
Assumptions on the weights. We assume that $T_{0}$ and $T_{1}$ in the definition of $\theta$ in (2.10) satisfy

$$
T_{0} \in\left(0, T_{0}^{*}\right), \quad T_{1} \in\left(0, T_{1}^{*}\right),
$$

where $T_{0}^{*}$ and $T_{1}^{*}$ are given by Lemma 3.1.

We also assume that the function $\psi$ in (2.7) can be extended in $[0, T] \times \mathbb{R}^{2}$ such that

$$
\psi \in C^{1}\left([0, T] \times \mathbb{R}^{2}\right) \quad \text { and } \quad \partial_{t} \psi+\overline{\mathbf{y}} \cdot \nabla \psi=0 \quad \text { in }(0, T) \times \mathbb{R}^{2} .
$$

Assumptions on $\widehat{\mathbf{u}}$. In order to derive estimates on $\rho$, we shall assume that $\widehat{\mathbf{u}}$ is in a weighted Sobolev space. According to Theorem 2.3, it is natural to assume

$$
\begin{aligned}
& \xi^{-2} \widehat{\mathbf{u}} e^{s \varphi} \in L^{2}\left(0, T ; \mathbf{L}^{2}(\Omega)\right), \quad \operatorname{div} \widehat{\mathbf{u}}=0 \quad \text { in } \Omega_{T}, \\
& \widehat{\mathbf{u}} e^{3 s \varphi^{*} / 4} \in L^{2}\left(0, T ; \mathbf{H}^{2}(\Omega)\right) \quad \text { with }\left\|\widehat{\mathbf{u}} e^{3 s \varphi^{*} / 4}\right\|_{L^{2}\left(0, T ; \mathbf{H}^{2}(\Omega)\right)} \leq \varsigma .
\end{aligned}
$$

Extension of $\widehat{\mathbf{u}}$. To fit into the setting of Section 3.2, we extend $\widehat{\mathbf{u}}$ on $[0, T] \times \mathbb{R}^{2}$ that we still denote the same: $\widehat{\mathbf{u}}=$ $\mathbf{E}(\widehat{\mathbf{u}})$, where $\mathbf{E}$ denotes an extension from $\mathbf{H}^{2}(\Omega)$ to $\mathbf{H}^{2}\left(\mathbb{R}^{2}\right)$ such that $\|\mathbf{E}(\mathbf{v})\|_{\mathbf{H}^{2}\left(\mathbb{R}^{2}\right)} \leq 2\|\mathbf{v}\|_{\mathbf{H}^{2}(\Omega)}$ for all $\mathbf{v} \in \mathbf{H}^{2}(\Omega)$. This allows us to define the flow $\widehat{X}$ by (3.2) for $(t, \tau, x) \in[0, T]^{2} \times \mathbb{R}^{2}$.

Note that, for $s$ large enough, this last assumption is stronger than (3.5) and is thus perfectly compatible with the construction of Section 3.2, as it implies in particular that

$$
\|\theta \widehat{\mathbf{u}}\|_{L^{2}\left(0, T ; \mathbf{L}^{\infty}\left(\mathbb{R}^{2}\right)\right)} \leq c \varsigma e^{-c_{0} s \lambda},
$$

where $c_{0}>0$ is independent of $s$ and $\lambda$. For the following we suppose that $s \geq s_{0}$ and $\lambda \geq 1$ with $s_{0}$ large enough such that (3.5) and (3.20) are satisfied.

On the flows $\widehat{X}$ and $\bar{X}$. We first establish a lemma on the closeness of $\widehat{X}$ to $\bar{X}$.

Lemma 3.3. There exists $c>0$ independent of $s$ and $\lambda$ such that for all $(\tau, t) \in[0, T]^{2}$ and $x \in \mathbb{R}^{2}$ :

$$
|\widehat{X}(\tau, t, x)-\bar{X}(\tau, t, x)| \leq c \varsigma e^{-c_{0} s \lambda} .
$$

Moreover, if $T_{0} \leq t \leq \tau \leq T$, we also have

$$
\theta(t)|\widehat{X}(\tau, t, x)-\bar{X}(\tau, t, x)| \leq c \varsigma e^{-c_{0} s \lambda}
$$

Proof. Estimate (3.21) is an immediate consequence of (3.4) and (3.20). From (3.4), we also have

$$
\theta(t)|\widehat{X}(\tau, t, x)-\bar{X}(\tau, t, x)| \leq T^{1 / 2} e^{L T} \theta(t)\|\widehat{\mathbf{u}}\|_{L^{2}\left(t, \tau ; \mathbf{L}^{\infty}\left(\mathbb{R}^{2}\right)\right)},
$$

where $L=\|\nabla \overline{\mathbf{y}}\|_{L^{\infty}\left(0, T ; \mathbf{L}^{\infty}\left(\mathbb{R}^{2}\right)\right)}$. Using the fact that $\theta$ is increasing on $\left[T_{0}, T\right]$,

$$
\theta(t)|\widehat{X}(\tau, t, x)-\bar{X}(\tau, t, x)| \leq T^{1 / 2} e^{L T}\|\theta \widehat{\mathbf{u}}\|_{L^{2}\left(t, \tau ; \mathbf{L}^{\infty}\left(\mathbb{R}^{2}\right)\right)},
$$

for all $T_{0} \leq t \leq \tau \leq T$, which concludes the proof of Lemma 3.3 by (3.20).

On the weight functions. Here, we shall deeply use the fact that $\psi$ is assumed to solve the transport equation (3.17), thus implying in particular that

$$
\forall(t, \tau, x) \in[0, T]^{2} \times \mathbb{R}^{2}, \quad \psi(t, \bar{X}(t, \tau, x))=\psi(\tau, x) .
$$

We then show the following lemma:

Lemma 3.4. There exist $c_{1}>0, c_{2}>0$ and $c_{3}>0$ independent of $s$ and $\lambda$, and $s_{0}>1$ such that for all $s \geq s_{0}, \lambda \geq 1$, the following inequalities hold:

1. For all $t \in\left[0, T-2 T_{1}\right], \tau \in[0, t]$ and $x \in \mathbb{R}^{2}$,

$$
\begin{aligned}
& \varphi(t, x)-\varphi(\tau, \widehat{X}(\tau, t, x)) \leq c_{1} \varsigma e^{-c_{2} s \lambda}, \\
& \frac{\xi(\tau, \widehat{X}(\tau, t, x))}{\xi(t, x)} \leq 2 e^{c_{1} \varsigma e^{-c_{2} s \lambda}} .
\end{aligned}
$$


2. For all $t \in\left[T_{0}, T\right], \tau \in[t, T]$ and $x \in \mathbb{R}^{2}$,

$$
\begin{aligned}
& \varphi(t, x)-\varphi(\tau, \widehat{X}(\tau, t, x)) \leq c_{1} \varsigma e^{-c_{2} s \lambda}-c_{3}(\theta(\tau)-\theta(t)), \\
& \frac{\xi(\tau, \widehat{X}(\tau, t, x))}{\xi(t, x)} \leq \frac{\theta(\tau)}{\theta(t)} e^{c_{1} \varsigma e^{-c_{2} s \lambda} .}
\end{aligned}
$$

Proof. We focus on the proof of item 2, the first one being similar and easier because $\theta$ takes value in [1,2] close to $t=0$. Estimate (3.26) follows from the following computations: for $T_{0} \leq t \leq \tau \leq T$,

$$
\begin{aligned}
\varphi(t, x)-\varphi(\tau, \widehat{X}(\tau, t, x)) \\
\quad=\theta(t)\left(\lambda e^{6 \lambda(m+1)}-e^{\lambda \psi(t, x)}\right)-\theta(\tau)\left(\lambda e^{6 \lambda(m+1)}-e^{\lambda \psi(\tau, \widehat{X}(\tau, t, x))}\right) \\
\quad=\theta(t)\left(e^{\lambda \psi(\tau, \widehat{X}(\tau, t, x))}-e^{\lambda \psi(t, x)}\right)+(\theta(t)-\theta(\tau))\left(\lambda e^{6 \lambda(m+1)}-e^{\lambda \psi(\tau, \widehat{X}(\tau, t, x))}\right) \\
\leq \theta(t)\left(e^{\lambda \psi(\tau, \widehat{X}(\tau, t, x))}-e^{\lambda \psi(t, x)}\right)-c_{3}(\theta(\tau)-\theta(t)),
\end{aligned}
$$

for some $c_{3}>0$, where we used in the last estimate that $\theta$ is increasing on $\left[T_{0}, T\right]$. We then use (3.23) and (3.22):

$$
\begin{aligned}
\left|\theta(t)\left(e^{\lambda \psi(\tau, \widehat{X}(\tau, t, x))}-e^{\lambda \psi(t, x)}\right)\right| & =\theta(t)\left|e^{\lambda \psi(\tau, \widehat{X}(\tau, t, x))}-e^{\lambda \psi(\tau, \bar{X}(\tau, t, x))}\right| \\
& \leq c \theta(t) \lambda\|\nabla \psi\|_{\infty} e^{\lambda(6 m+1)}|\widehat{X}(\tau, t, x)-\bar{X}(\tau, t, x)| \leq c_{1} \zeta e^{-c_{2} s \lambda},
\end{aligned}
$$

for $s$ large enough, as announced in (3.26). Next, by construction we have

$$
\begin{aligned}
\frac{\xi(\tau, \widehat{X}(\tau, t, x))}{\xi(t, x)} & =\frac{\theta(\tau)}{\theta(t)} e^{\lambda(\psi(\tau, \widehat{X}(\tau, t, x))-\psi(\tau, \bar{X}(\tau, t, x)))} \\
& \leq \frac{\theta(\tau)}{\theta(t)} e^{\lambda\|\nabla \psi\|_{\infty}|\widehat{X}(\tau, t, x)-\bar{X}(\tau, t, x)|},
\end{aligned}
$$

which immediately yields (3.27) by (3.22).

We immediately deduce from Lemma 3.4 the following:

Proposition 3.5. Introducing the weight function

$$
\aleph(t, x): \stackrel{\text { def }}{=}(\xi(t, x))^{-2} e^{s \varphi(t, x)},
$$

there exist $s_{0} \geq 1$ and $c>0$ independent of $s$ and $\lambda$ such that for all $\lambda \geq 1, s \geq s_{0}$, for all $(\tau, t, x) \in[0, T] \times[0, T] \times \bar{\Omega}$ satisfying $\tau \leq t \leq T-2 T_{1}$ or $T_{0} \leq t \leq \tau$,

$$
\aleph(t, x) \leq c \aleph(\tau, \widehat{X}(\tau, t, x)) .
$$

Proof. If $\tau \leq t \leq T-2 T_{1}$ then (3.30) follows immediately from (3.24) and (3.25).

If $T_{0} \leq t \leq \tau$ then (3.30) follows from (3.26) and (3.27):

$$
\aleph(t, x) \leq\left(\frac{\theta^{2}(\tau) e^{-c_{3} s \theta(\tau)}}{\theta^{2}(t) e^{-c_{3} s \theta(t)}}\right) e^{c_{1} \varsigma(s+2) e^{-s \lambda c_{2}}} \aleph(\tau, \widehat{X}(\tau, t, x)) .
$$

But, for $s \geq 2 / c_{3}$, the function $x \mapsto x^{2} e^{-c_{3} s x}$ is decreasing on $[1,+\infty)$ and then, since $\theta$ is increasing on $\left[T_{0}, T\right]$, $\theta^{2}(\tau) e^{-c_{3} s \theta(\tau)} \leq \theta^{2}(t) e^{-c_{3} s \theta(t)}$.

On the controlled trajectory $\rho$. We now derive estimates on the controlled trajectory $\rho$ given by Section 3.2:

Theorem 3.6. Let $\psi, \theta, \varphi, \xi$ are defined in (2.7)-(2.11) and assume (3.16), (3.17). Further assume that $\widehat{\mathbf{u}}$ satisfies (3.18) and (3.19) with $s \geq s_{0}, \lambda \geq 1$ and $s_{0}$ large enough such that (3.5) and (3.20) are satisfied. 
There exists $c>0$ independent of $s, \lambda$ and $\widehat{\mathbf{u}}$ such that the solution $\rho$ given by Section 3.2 satisfies

$$
\|\aleph \rho\|_{L^{2}\left(\Omega_{T}\right)} \leq C\left(\|\aleph \widehat{\mathbf{u}}\|_{L^{2}\left(0, T ; \mathbf{L}^{2}(\Omega)\right)}+e^{s \varphi^{*}(0)}\left\|\rho_{0}\right\|_{L^{2}(\Omega)}\right),
$$

where $\aleph$ is given by (3.29), and

$$
\left\|e^{s \lambda e^{6 \lambda(m+1)} \theta(t) / 2} \rho\right\|_{L^{\infty}\left(\Omega_{T}\right)} \leq C\left(\left\|e^{s \lambda e^{6 \lambda(m+1)} \theta(t) / 2} \widehat{\mathbf{u}}\right\|_{L^{2}\left(0, T ; \mathbf{L}^{\infty}(\Omega)\right)}+e^{s \lambda e^{6 \lambda(m+1)}}\left\|\rho_{0}\right\|_{L^{\infty}(\Omega)}\right) .
$$

Proof. The proof of Theorem 3.6 follows from the precise description of $\rho_{f}$ and $\rho_{b}$ given in (3.14)-(3.15).

Let us begin with the proof of estimate (3.32). On one hand, as $t \mapsto s \lambda e^{6 \lambda(m+1)} \theta(t)$ is non-increasing on $(0, T-$ $\left.2 T_{1}\right)$, from (3.14) we get, for all $(t, x) \in\left(0, T-2 T_{1}\right) \times \Omega$

$$
e^{s \lambda e^{6 \lambda(m+1)} \theta(t)}\left|\rho_{f}(t, x)\right|^{2} \leq 2 e^{s \lambda e^{6 \lambda(m+1)} \theta(t)}\left\|\rho_{0}\right\|_{L^{\infty}(\Omega)}^{2}+2\|\nabla \bar{\sigma}\|_{\mathbf{L}^{\infty}\left(\Omega_{T}\right)}^{2} \int_{0}^{t} e^{s \lambda e^{6 \lambda(m+1)} \theta(\tau)}\|\widehat{\mathbf{u}}(\tau, \cdot)\|_{\mathbf{L}^{\infty}(\Omega)}^{2} \mathrm{~d} \tau .
$$

On the other hand, using that $t \mapsto s \lambda e^{6 \lambda(m+1)} \theta(t)$ is non-decreasing on $\left(T_{0}, T\right)$, from (3.15), similarly, we have, for all $(t, x) \in\left(T_{0}, T\right) \times \Omega$,

$$
e^{s \lambda e^{6 \lambda(m+1)} \theta(t)}\left|\rho_{b}(t, x)\right|^{2} \leq\|\nabla \bar{\sigma}\|_{\mathbf{L}^{\infty}\left(\Omega_{T}\right)}^{2} \int_{t}^{T} e^{s \lambda e^{6 \lambda(m+1)} \theta(\tau)}\|\widehat{\mathbf{u}}(\tau, \cdot)\|_{\mathbf{L}^{\infty}(\Omega)}^{2} \mathrm{~d} \tau .
$$

Together with the fact that the solution $\chi$ of (3.8) takes value in $[0,1]$ on $\Omega_{T}$ and the properties (3.10), these two estimates easily yield (3.32).

We then focus on the proof of (3.31), that mainly relies on the two following estimates: for all time $t \in\left(0, T-2 T_{1}\right)$, we get

$$
\int_{\Omega}\left|\rho_{f}(t)\right|^{2} \aleph^{2}(t) \mathrm{d} x \leq C\left(e^{2 s \varphi^{*}(0)} \int_{\Omega}\left|\rho_{0}\right|^{2} \mathrm{~d} x+\iint_{\Omega_{T}}|\widehat{\mathbf{u}}|^{2} \aleph^{2} \mathrm{~d} x \mathrm{~d} \tau\right),
$$

and for all time $t \in\left(T_{0}, T\right)$,

$$
\int_{\Omega}\left|\rho_{b}(t)\right|^{2} \aleph^{2}(t) \mathrm{d} x \leq c \iint_{\Omega_{T}}|\widehat{\mathbf{u}}|^{2} \aleph^{2} \mathrm{~d} x \mathrm{~d} \tau .
$$

Indeed, once estimates (3.33)-(3.34) are proved, we can bound the $L^{2}\left(\Omega_{T}\right)$-norm of $\aleph \rho$ by the sum of the $L^{\infty}((0, T-$ $\left.\left.2 T_{1}\right) ; L^{2}(\Omega)\right)$-norm of $\rho_{f}$ and of the $L^{\infty}\left(\left(T_{0}, T\right) ; L^{2}(\Omega)\right)$-norm of $\rho_{b}$, and estimate (3.31) immediately follows.

Let us first present the proof of (3.33). We fix $t \in\left[0, T-2 T_{1}\right]$. From (3.14) and (3.30) we deduce that, for $x \in$ $\Omega_{[0]}(t)$,

$$
\left|\rho_{f}(t, x)\right|^{2} \aleph^{2}(t, x) \leq C\left(\left|\rho_{0}(\widehat{X}(0, t, x))\right|^{2} \aleph^{2}(0, \widehat{X}(0, t, x))+\int_{0}^{t}|\widehat{\mathbf{u}}(\tau, \widehat{X}(\tau, t, x))|^{2} \aleph^{2}(\tau, \widehat{X}(\tau, t, x)) \mathrm{d} \tau\right),
$$

whereas for $x \in \Omega \backslash \Omega_{[0]}(t)$,

$$
\left|\rho_{f}(t, x)\right|^{2} \aleph^{2}(t, x) \leq C \int_{t_{\text {in }}(t, x)}^{t}|\widehat{\mathbf{u}}(\tau, \widehat{X}(\tau, t, x))|^{2} \aleph^{2}(\tau, \widehat{X}(\tau, t, x)) \mathrm{d} \tau .
$$

Combining these two estimates, for all $t \in\left(0, T-2 T_{1}\right)$ we get:

$$
\begin{aligned}
\int_{\Omega}\left|\rho_{f}(t, x)\right|^{2} \aleph^{2}(t, x) \mathrm{d} x \leq & C \int_{\Omega_{[0]}(t)}\left|\rho_{0}(\widehat{X}(0, t, x))\right|^{2} \aleph^{2}(0, \widehat{X}(0, t, x)) \mathrm{d} x \\
& +C \int_{0}^{t} \int_{\Omega} \mathbf{1}_{\left[t_{\mathrm{in}}(t, x), t\right]}(\tau)|\widehat{\mathbf{u}}(\tau, \widehat{X}(\tau, t, x))|^{2} \aleph^{2}(\tau, \widehat{X}(\tau, t, x)) \mathrm{d} x \mathrm{~d} \tau .
\end{aligned}
$$


Since $\overline{\mathbf{y}}+\widehat{\mathbf{u}}$ is divergence free in $\Omega_{T}$, the Jacobian of $x \mapsto \widehat{X}(t, \tau, x)$ equals 1 identically. Therefore,

$$
\int_{\Omega_{[0]}(t)}\left|\rho_{0}(\widehat{X}(0, t, x))\right|^{2} \aleph^{2}(0, \widehat{X}(0, t, x)) \mathrm{d} x=\int_{\widehat{X}\left(0, t, \Omega_{[0]}(t)\right)}\left|\rho_{0}(x)\right|^{2} \aleph^{2}(0, x) \mathrm{d} x \leq \int_{\Omega}\left|\rho_{0}(x)\right|^{2} \aleph^{2}(0, x) \mathrm{d} x .
$$

Similarly, we get

$$
\int_{0}^{t} \int_{\Omega} \mathbf{1}_{\left[t_{\mathrm{in}}(t, x), t\right]}(\tau)|\widehat{\mathbf{u}}(\tau, \widehat{X}(\tau, t, x))|^{2} \aleph^{2}(\tau, \widehat{X}(\tau, t, x)) \mathrm{d} x \mathrm{~d} \tau \leq \int_{0}^{t} \int_{\Omega}|\widehat{\mathbf{u}}(\tau, x)|^{2} \aleph^{2}(\tau, x) \mathrm{d} \tau \mathrm{d} x
$$

Estimate (3.33) then follows from (3.35).

The proof of (3.34) is based on (3.15) and follows the same lines. It is therefore left to the reader.

\section{Proof of Theorem 1.1}

We are now in position to prove Theorem 1.1. The idea is to construct suitable convex sets which are invariant by the mapping $\mathscr{F}=\mathscr{F}\left(\rho_{0}, \mathbf{u}_{0}\right)$ in $(1.21)$ and relatively compact for a topology making $\mathscr{F}$ continuous. In all this section, we assume the assumptions of Theorem 1.1.

\subsection{Main steps of the proof of Theorem 1.1}

In the introduction, we introduced formally a mapping $\mathscr{F}$. We are now in position to define it precisely.

In order to do this, the first step in the proof of Theorem 1.1 is to construct a weight function $\tilde{\psi}$ which is suitable for both Section 2 and Section 3, i.e. suitable in the same time for controlling the velocity equation and the density equation. We claim the following result, proved in Section 4.2:

Lemma 4.1. Let $\Omega$ be a smooth bounded domain. Further assume the regularity condition (1.10) on $(\bar{\sigma}, \overline{\mathbf{y}})$, the geometric condition (1.8) and condition (1.9).

Then one can find a smooth $\left(C^{2}\right)$ bounded domain $\mathcal{O}$ satisfying (2.1) such that there exists a $C^{2}([0, T] \times$ $\mathbb{R}^{2}$ )-function $\tilde{\psi}$ satisfying the transport equation (3.17) for some extension $\overline{\mathbf{y}}$ of $\overline{\mathbf{y}}$ in $[0, T] \times \mathbb{R}^{2}$ and satisfying assumptions (2.7)-(2.8) for $\omega_{T}=[0, T] \times \omega$ and $\tilde{\omega}_{T}=[0, T] \times \tilde{\omega}$ where $\omega, \tilde{\omega}$ are two subdomains of $\mathcal{O} \backslash \bar{\Omega}$ such that $\tilde{\omega} \Subset \omega$.

We then consider the extension $\overline{\mathbf{y}}$ given by Lemma 4.1. Next, we take $T_{0}^{*}, T_{1}^{*}$ and $\varsigma>0$ given by Lemma 3.2 and fix $T_{0} \in\left(0, T_{0}^{*}\right)$ and $T_{1} \in\left(0, T_{1}^{*}\right)$. We then use the function $\psi, \theta, \varphi$ and $\xi$ given by (2.9), (2.10), (2.11) for $m \geq 5$, $s \geq s_{0}, \lambda \geq \lambda_{0}$, and the notations given in (2.14)-(2.15). Moreover, we suppose that $s_{0}, \lambda_{0}$ are large enough given by Theorem 2.3 and Theorem 3.6. Now, we define the spaces $\mathbf{X}_{s, \lambda}$ and $Y_{s, \lambda}$ depending on positive parameters $s \geq s_{0}$ and $\lambda \geq \lambda_{0}$ as follows:

$$
\begin{aligned}
& \mathbf{X}_{s, \lambda}: \stackrel{\text { def }}{=}\left\{\mathbf{u} \in \mathbf{L}^{2}\left(\Omega_{T}\right), \text { with } \operatorname{div}(\mathbf{u})=0 \text { in } \Omega_{T},\right. \\
& s^{1 / 4} \xi^{1 / m-2} e^{s \varphi} \mathbf{u} \in \mathbf{L}^{2}\left(\Omega_{T}\right), \\
&\left.e^{3 s \varphi^{*} / 4} \mathbf{u} \in L^{2}\left(0, T ; \mathbf{H}^{2}(\Omega)\right) \cap H^{1}\left(0, T ; \mathbf{L}^{2}(\Omega)\right)\right\},
\end{aligned}
$$

endowed with the norm

$$
\|\mathbf{u}\|_{\mathbf{X}_{s, \lambda}}^{2}: \stackrel{\text { def }}{=}\left\|e^{3 s \varphi^{*} / 4} \mathbf{u}\right\|_{L^{2}\left(\mathbf{H}^{2}\right) \cap H^{1}\left(\mathbf{L}^{2}\right)}^{2}+s^{1 / 2}\left\|\xi^{1 / m-2} e^{s \varphi} \mathbf{u}\right\|_{\mathbf{L}^{2}\left(\Omega_{T}\right)}^{2},
$$

and

$$
Y_{s, \lambda}: \stackrel{\text { def }}{=}\left\{\rho \in L^{\infty}\left(\Omega_{T}\right), \text { with } \xi^{-2} e^{s \varphi} \rho \in L^{2}\left(\Omega_{T}\right) \text { and } e^{s \lambda e^{6 \lambda(m+1)} \theta / 2} \rho \in L^{\infty}\left(\Omega_{T}\right)\right\},
$$

endowed with the norm

$$
\|\rho\|_{Y_{s, \lambda}}: \stackrel{\text { def }}{=}\left\|\xi^{-2} e^{s \varphi} \rho\right\|_{L^{2}\left(\Omega_{T}\right)}+\left\|e^{s \lambda e^{6 \lambda(m+1)} \theta / 2} \rho\right\|_{L^{\infty}\left(\Omega_{T}\right)} .
$$


We also introduce the space $\mathbf{F}_{s, \lambda}$ defined by

$$
\begin{gathered}
\mathbf{F}_{s, \lambda}: \stackrel{\text { def }}{=}\left\{\mathbf{f} \in L^{2}\left(0, T ; \mathbf{L}^{2}(\Omega)\right), \text { with } \xi^{-2} \mathbf{f} e^{s \varphi} \in L^{2}\left(0, T ; \mathbf{L}^{2}(\Omega)\right)\right\} \\
\text { endowed with the norm }\|\mathbf{f}\|_{\mathbf{F}_{s, \lambda}}: \stackrel{\text { def }}{=}\left\|\xi^{-2} \mathbf{f} e^{s \varphi}\right\|_{L^{2}\left(\mathbf{L}^{2}\right)} .
\end{gathered}
$$

Note that, in the above definitions as well as in the following results, we keep the dependence in both parameters $\lambda$ and $s$ to be consistent with notations of Section 2. However, only the dependence in $s$ will be needed in this section.

We then derive the following results.

Theorem 4.2 (On the mapping $\mathscr{F}_{1}$ ). Fix $\rho_{0} \in L^{\infty}(\Omega)$. For all $\widehat{\mathbf{u}} \in \mathbf{X}_{s, \lambda}$ with $\|\widehat{\mathbf{u}}\|_{\mathbf{X}_{s, \lambda}} \leq \varsigma$, the construction in Section 3.2 yields $\rho=\mathscr{F}_{1}\left(\widehat{\mathbf{u}}, \rho_{0}\right)$ solution of the control problem (1.19). Besides, $\rho \in Y_{s, \lambda}$ and for some constant $C$ independent of $s \geq s_{0}$ and $\lambda \geq \lambda_{0}$,

$$
\|\rho\|_{Y_{s, \lambda}} \leq C\left(\frac{1}{s^{1 / 4}}\|\widehat{\mathbf{u}}\|_{\mathbf{X}_{s, \lambda}}+e^{s \varphi^{*}(0)}\left\|\rho_{0}\right\|_{L^{\infty}(\Omega)}\right) .
$$

Furthermore, the application $\mathscr{F}_{1}$ satisfies the following compactness property: If $\widehat{\mathbf{u}}_{n}$ is a sequence of functions in $\mathbf{X}_{s, \lambda}$ with $\left\|\widehat{\mathbf{u}}_{n}\right\|_{\mathbf{X}_{s, \lambda}} \leq \varsigma$ which weakly converges to some $\widehat{\mathbf{u}}$ in $X_{s, \lambda}$, the corresponding sequence $\rho_{n}=\mathscr{F}_{1}\left(\widehat{\mathbf{u}}_{n}, \rho_{0}\right)$ strongly converges to $\mathscr{F}_{1}\left(\widehat{\mathbf{u}}, \rho_{0}\right)$ in all $L^{q}\left(\Omega_{T}\right)$ for $q \in[1, \infty)$.

The proof of Theorem 4.2 is done in Section 4.3. Let us point out that the compactness property stated in Theorem 4.2 is of primary importance for our result and follows from [3, Theorem 4].

We then focus on the study of the mapping $\mathscr{F}_{2}$ :

Theorem 4.3 (On the mapping $\mathscr{F}_{2}$ ). We can define a bounded linear mapping $\mathscr{F}_{2}: \mathbf{F}_{s, \lambda} \times \mathbf{V}_{0}^{1}(\Omega) \rightarrow \mathbf{X}_{s, \lambda}$ such that for all $\mathbf{u}_{0} \in \mathbf{V}_{0}^{1}(\Omega)$ and $\mathbf{f} \in \mathbf{F}_{s, \lambda}, \mathbf{u}=\mathscr{F}_{2}\left(\mathbf{f}, \mathbf{u}_{0}\right)$ solves the control problem (1.20) and satisfies, for some constant $C>0$ independent of $s \geq s_{0}$ and $\lambda \geq \lambda_{0}$,

$$
\|\mathbf{u}\| \mathbf{X}_{s, \lambda} \leq C\left(\|\mathbf{f}\|_{\mathbf{F}_{s, \lambda}}+e^{\frac{5}{4} s \varphi^{*}(0)}\left\|\mathbf{u}_{0}\right\|_{\mathbf{H}_{0}^{1}(\Omega)}\right) .
$$

Theorem 4.3 is a direct consequence of Theorem 2.3: the mapping $\mathscr{F}_{2}$ is obtained by restricting the controlled trajectory given by Theorem 2.3 to $(0, T) \times \Omega$. Of course, this depends on the extension $\mathcal{O}$ of $\Omega$, but this choice is done once for all. Estimate (4.3) is then a rewriting of Theorem 2.3 by taking into account that $\mathbf{f}$ and $\mathbf{u}_{0}$ are extended by zero outside $\Omega$.

We are then able to derive the following properties on the mapping $\mathscr{F}$ in (1.21), whose proof is postponed to Section 4.4:

Theorem 4.4. Let $\rho_{0} \in L^{\infty}(\Omega)$ and $\mathbf{u}_{0} \in \mathbf{V}_{0}^{1}(\Omega)$.

Then for all $s \geq s_{0}$ and $\lambda \geq \lambda_{0}$ the mapping $\mathscr{F}$ in (1.21) is well-defined for all $\widehat{\mathbf{u}} \in \mathbf{X}_{s, \lambda}$ with $\|\widehat{\mathbf{u}}\|_{\mathbf{X}_{s, \lambda}} \leq \varsigma$. Besides, for all $\widehat{\mathbf{u}} \in \mathbf{X}_{s, \lambda}$ with $\|\widehat{\mathbf{u}}\|_{\mathbf{X}_{s, \lambda}} \leq \varsigma, \mathbf{u}=\mathscr{F}(\widehat{\mathbf{u}})$ belongs to $\mathbf{X}_{s, \lambda}$, and satisfies, for some constant $C_{0}$ independent of $s$ and $\lambda$,

$$
\|\mathbf{u}\|_{\mathbf{X}_{s, \lambda}} \leq C_{0}\left(\frac{1}{s^{1 / 4}}\|\widehat{\mathbf{u}}\|_{\mathbf{X}_{s, \lambda}}+\|\widehat{\mathbf{u}}\|_{\mathbf{X}_{s, \lambda}}^{2}+e^{s \varphi^{*}(0)}\left\|\rho_{0}\right\|_{L^{\infty}(\Omega)}+e^{2 s \varphi^{*}(0)}\left\|\rho_{0}\right\|_{L^{\infty}(\Omega)}^{2}+e^{\frac{5}{4} s \varphi^{*}(0)}\left\|\mathbf{u}_{0}\right\|_{\mathbf{H}_{0}^{1}(\Omega)}\right)
$$

Moreover, if $\widehat{\mathbf{u}}_{n}$ is a sequence of functions in $\mathbf{X}_{s, \lambda}$ with $\left\|\widehat{\mathbf{u}}_{n}\right\| \mathbf{X}_{s, \lambda} \leq \varsigma$ which weakly converges to some $\widehat{\mathbf{u}}$ in $\mathbf{X}_{s, \lambda}$, the corresponding sequence $\mathbf{u}_{n}=\mathscr{F}\left(\widehat{\mathbf{u}}_{n}\right)$ strongly converges to $\mathbf{u}=\mathscr{F}(\widehat{\mathbf{u}})$ in $L^{2}\left(0, T ; \mathbf{L}^{2}(\Omega)\right)$.

We may then conclude the proof of Theorem 1.1. For $R \in(0, \varsigma)$, we introduce the closed convex set

$$
\mathbf{X}_{s, \lambda}^{R}=\left\{\mathbf{u} \in \mathbf{X}_{s, \lambda} \text { with }\|\mathbf{u}\|_{\mathbf{X}_{s, \lambda}} \leq R\right\} .
$$

We then choose $R$ small enough such that $C_{0} R \leq 1 / 4$, where $C_{0}$ is the constant in (4.4), $\lambda=\lambda_{0}$ and $s \geq s_{0}$ large enough to guarantee $C_{0} \leq s^{1 / 4} / 4$. We then get from (4.4) that for all $\widehat{\mathbf{u}} \in \mathbf{X}_{s, \lambda_{0}}^{R}, \mathbf{u}=\mathscr{F}(\widehat{\mathbf{u}})$ satisfies 


$$
\|\mathbf{u}\| \mathbf{X}_{s, \lambda_{0}} \leq \frac{R}{2}+C_{0}\left(e^{s \varphi^{*}(0)}\left\|\rho_{0}\right\|_{L^{\infty}(\Omega)}+e^{2 s \varphi^{*}(0)}\left\|\rho_{0}\right\|_{L^{\infty}(\Omega)}^{2}+e^{\frac{5}{4} s \varphi^{*}(0)}\left\|\mathbf{u}_{0}\right\|_{\mathbf{H}_{0}^{1}(\Omega)}\right) .
$$

Thus, choosing $\varepsilon>0$ sufficiently small in (1.11), we can guarantee that the mapping $\mathscr{F}$ maps $\mathbf{X}_{s, \lambda_{0}}^{R}$ to itself.

We then check that the set $\mathbf{X}_{s, \lambda_{0}}^{R}$ is compact in $L^{2}\left(0, T ; \mathbf{L}^{2}(\Omega)\right)$ as $H^{1}\left(0, T ; \mathbf{L}^{2}(\Omega)\right) \cap L^{2}\left(0, T ; \mathbf{H}^{2}(\Omega)\right)$ is compactly embedded in $L^{2}\left(0, T ; \mathbf{L}^{2}(\Omega)\right)$ due to Rellich's compactness theorem and Aubin-Lions' theorem.

Besides, the mapping $\mathscr{F}$ is continuous on $\mathbf{X}_{s, \lambda_{0}}^{R}$ endowed with the $L^{2}\left(0, T ; \mathbf{L}^{2}(\Omega)\right)$-topology from Theorem 4.4. Indeed, if $\widehat{\mathbf{u}}_{n}$ is a sequence of functions in $\mathbf{X}_{s, \lambda_{0}}^{R}$ which strongly converges to $\widehat{\mathbf{u}}$ in $L^{2}\left(0, T ; \mathbf{L}^{2}(\Omega)\right)$, it necessarily weakly converges in $\mathbf{X}_{s, \lambda_{0}}^{R}$. Thus, from the last item of Theorem 4.4, $\mathbf{u}_{n}=\mathscr{F}\left(\widehat{\mathbf{u}}_{n}\right)$ strongly converges to $\mathbf{u}$ in $L^{2}\left(0, T ; \mathbf{L}^{2}(\Omega)\right)$.

Schauder's fixed point theorem then implies the existence of a fixed point to the mapping $\mathscr{F}$, and concludes the proof of Theorem 1.1.

\subsection{Proof of Lemma 4.1}

We do it in several steps.

Construction of $\mathcal{O}$. In a neighborhood of $\Gamma_{c}$, according to Assumption (1.9), there exists a $C^{2}$ extension $\mathcal{O}$ of $\Omega$ such that

- $\Omega \subset \mathcal{O}$;

- $\Gamma_{0} \subset \partial \Omega \cap \partial \mathcal{O}$ and for all $t \in(0, T)$ and $x \in \partial \Omega \cap \partial \mathcal{O}, \overline{\mathbf{y}}(t, x) \cdot \mathbf{n} \geq \gamma / 2$;

- $\partial \mathcal{O} \cap \partial \Omega$ and $\mathcal{O} \backslash \bar{\Omega}$ have a finite number of connected components.

Let $\omega, \tilde{\omega}$ be two subdomains of $\mathcal{O} \backslash \bar{\Omega}$ such that $\tilde{\omega} \Subset \omega$ and fix $d_{0}=\operatorname{dist}(\tilde{\omega}, \Omega)$.

Construction of an extension $\overline{\mathbf{y}}_{e}$ of $\overline{\mathbf{y}}$ in $[0, T] \times \mathbb{R}^{2}$. We then construct an extension $\overline{\mathbf{y}}_{e} \in \mathbf{C}^{2}\left([0, T] \times \mathbb{R}^{2}\right)$ of $\overline{\mathbf{y}}$ outside $\Omega_{T}$ (i.e. $\overline{\mathbf{y}}_{e} \equiv \overline{\mathbf{y}}$ in $\Omega_{T}$ ) satisfying

$$
\left\|\overline{\mathbf{y}}_{e}\right\|_{\mathbf{C}^{2}([0, T] \times \overline{\mathcal{O}})}<\infty, \quad \inf _{[0, T] \times \partial \mathcal{O}} \overline{\mathbf{y}}_{e} \cdot \mathbf{n}>0,
$$

and

$$
\overline{\mathbf{y}}_{e} \equiv 0 \quad \text { in }(0, T) \times \tilde{\omega} .
$$

Before going into the detailed construction of $\overline{\mathbf{y}}_{e}$, let us remark that $\overline{\mathbf{y}}_{e}$ cannot be divergence free as it would not be compatible with the condition $\inf _{[0, T] \times \partial \mathcal{O}} \overline{\mathbf{y}}_{e} \cdot \mathbf{n}>0$.

In order to construct such extension $\overline{\mathbf{y}}_{e}$, we proceed as follows. First, we consider any extension of $\overline{\mathbf{y}}$ in $\mathbf{C}^{2}([0, T] \times$ $\left.\mathbb{R}^{2}\right)$. By continuity, there exists $d_{1}>0$ such that for all $(t, x) \in(0, T) \times \partial \mathcal{O}$ with $d(x, \Omega)<d_{1}, \overline{\mathbf{y}}(t, x) \cdot \mathbf{n} \geq \gamma / 3$. We also introduce a function $\mathbf{m}$ in $\mathbf{C}^{2}\left([0, T] \times \mathbb{R}^{2}\right)$ such that $\mathbf{m} \cdot \mathbf{n}=1$ on the whole boundary $\partial \mathcal{O}$ and $\mathbf{m} \equiv 0$ in $\tilde{\omega}$, and a smooth non-negative cut-off function $\eta=\eta(x)$ taking value 1 in $\bar{\Omega}$ and 0 for all $x \in \mathcal{O}$ with $d(x, \Omega)>\min \left\{d_{0}, d_{1}\right\}$, and we then consider

$$
\overline{\mathbf{y}}_{e}(t, x)=\eta(x) \overline{\mathbf{y}}(t, x)+(1-\eta(x)) \mathbf{m}(x) .
$$

This function indeed belongs to $\mathbf{C}^{2}\left([0, T] \times \mathbb{R}^{2}\right)$. Besides,

$$
\inf _{[0, T] \times \partial \mathcal{O}} \overline{\mathbf{y}}_{e} \cdot \mathbf{n} \geq \min \left\{\frac{\gamma}{3}, 1\right\},
$$

and (4.6) is trivially satisfied as $\mathbf{m} \equiv 0$ and $\eta \equiv 0$ in $\tilde{\omega}$.

Construction of $\tilde{\psi}$ in $[0, T] \times \mathcal{O}$. We then construct a function $\widehat{\psi}_{T}=\widehat{\psi}_{T}(x)$ such that

- $\widehat{\psi}_{T}$ is a non-negative $C^{2}(\overline{\mathcal{O}})$ function;

- The critical points of $\widehat{\psi}_{T}$ all belong to $\tilde{\omega}$; 
- $\widehat{\psi}_{T}$ satisfies the following conditions on the boundary $\partial \mathcal{O}$ :

$$
\begin{cases}\widehat{\psi}_{T}(x)=0 & \text { on } \partial \mathcal{O} \\ \overline{\mathbf{y}}_{e}(T, x) \cdot \nabla \widehat{\psi}_{T}(x)=-1 & \text { on } \partial \mathcal{O} \\ \partial_{t} \overline{\mathbf{y}}_{e}(T, x) \cdot \nabla \widehat{\psi}_{T}(x)-\left(\overline{\mathbf{y}}_{e}(T, x) \cdot \nabla\right)^{2} \widehat{\psi}_{T}(x)=0 & \text { on } \partial \mathcal{O}\end{cases}
$$

- $\inf _{\mathcal{O}} \widehat{\psi}_{T}=\left(\widehat{\psi}_{T}\right)_{\mid \partial \mathcal{O}}=0$.

Note that such function exists according to the construction of Fursikov and Imanuvilov in [17] suitably modified to handle the conditions on the first and second order derivatives on the boundary of $\mathcal{O}$. This can be done easily following the lines of [27, Appendix III].

We then consider the solution $\widehat{\psi}$ of

$$
\begin{cases}\partial_{t} \widehat{\psi}+\overline{\mathbf{y}}_{e} \cdot \nabla \widehat{\psi}=0 & \text { in } \mathcal{O}_{T}, \\ \widehat{\psi}(t, x)=t-T & \text { on } \Gamma_{T}, \\ \widehat{\psi}(T)=\widehat{\psi}_{T} & \text { in } \mathcal{O} .\end{cases}
$$

Note that this problem is well-posed as, by construction, $\overline{\mathbf{y}}_{e}(t, x) \cdot \mathbf{n}>0$ for all $(t, x) \in(0, T) \times \partial \mathcal{O}$. We then want to check that

- $\partial_{\mathbf{n}} \widehat{\psi}(t, x) \leq 0$ for $(t, x) \in(0, T) \times \partial \mathcal{O}$;

- $\widehat{\psi}$ belongs to $C^{2}([0, T] \times \overline{\mathcal{O}})$;

- For all $t \in[0, T]$, the critical points of $\widehat{\psi}(t, \cdot)$ belong to $\tilde{\omega}$;

- For all $t \in[0, T], \inf _{\mathcal{O}} \widehat{\psi}(t, \cdot)=\widehat{\psi}(t)_{\mid \partial \mathcal{O}}$;

Using Eq. (4.8) and the fact that tangential derivatives of $\widehat{\psi}$ vanish due to the boundary conditions, we get, for all $(t, x) \in(0, T) \times \partial \mathcal{O}$,

$$
\overline{\mathbf{y}}_{e}(t, x) \cdot \mathbf{n} \partial_{\mathbf{n}} \widehat{\psi}(t, x)=-\partial_{t} \widehat{\psi}(t, x)=-1 .
$$

Using (4.5), we thus deduce that

$$
\forall(t, x) \in(0, T) \times \partial \mathcal{O}, \quad \partial_{\mathbf{n}} \widehat{\psi}(t, x) \leq \frac{-1}{\inf _{[0, T] \times \partial \mathcal{O}} \overline{\mathbf{y}}_{e}(t, x) \cdot \mathbf{n}}<0 .
$$

To describe more precisely the function $\widehat{\psi}$, we will introduce the flow $\bar{X}_{e}$ corresponding to $\overline{\mathbf{y}}_{e}$, i.e. the solution of

$$
\forall(t, \tau, x) \in[0, T]^{2} \times \mathbb{R}^{2}, \quad \partial_{t} \bar{X}_{e}(t, \tau, x)=\overline{\mathbf{y}}_{e}\left(t, \bar{X}_{e}(t, \tau, x)\right), \quad \bar{X}_{e}(\tau, \tau, x)=x .
$$

The fact that $\widehat{\psi} \in C^{2}([0, T] \times \overline{\mathcal{O}})$ follows from the following lemma, whose proof is postponed to Appendix B:

Lemma 4.5. Under the above assumptions, $\widehat{\psi} \in C^{2}([0, T] \times \overline{\mathcal{O}})$.

We then have to check that the critical points of $\widehat{\psi}(t, \cdot)$ all belong to $\tilde{\omega}$.

We first remark that (4.9) implies that there is no critical point on the boundary $\partial \mathcal{O}$. We then remark that $\nabla \widehat{\psi}$ solves the equation

$$
\partial_{t} \nabla \widehat{\psi}+\left(\overline{\mathbf{y}}_{e} \cdot \nabla\right) \nabla \widehat{\psi}+D \overline{\mathbf{y}}_{e} \nabla \widehat{\psi}=0 \quad \text { in } \mathcal{O}_{T} .
$$

From Eq. (4.11), if the point $x_{c}$ is a critical point for $\widehat{\psi}\left(t_{c}, \cdot\right)$, then for all $t$ in a neighborhood around $t_{c}, \bar{X}_{e}\left(t, t_{c}, x_{c}\right)$ is a critical point for $\widehat{\psi}(t, \cdot)$. This neighborhood actually corresponds to the set $I_{c}$ of time $t \in[0, T]$ such that the trajectory $\tau \mapsto \bar{X}_{e}\left(\tau, t_{c}, x_{c}\right)$ stays in $\mathcal{O}$ for $\tau$ between $t$ and $t_{c}$.

Since there is no critical point on the boundary $\partial \mathcal{O}$ and thanks to conditions (4.5), for all time $t_{c} \in[0, T]$, the critical points $x_{c}$ of $\widehat{\psi}\left(t_{c}, \cdot\right)$ are linked by a trajectory $\tau \mapsto \bar{X}_{e}\left(\tau, t_{c}, x_{c}\right)$ to a critical point $x_{c, T}$ of $\widehat{\psi}_{T}$, that is $x_{c}=$ $\bar{X}_{e}\left(t_{c}, T, x_{c, T}\right)$. By construction of $\widehat{\psi}_{T}, x_{c, T}$ necessarily belongs to $\tilde{\omega}$. But, according to condition (4.6), as long as $\bar{X}_{e}\left(t, T, x_{c, T}\right) \in \tilde{\omega}$, 


$$
\partial_{t} \bar{X}_{e}\left(t, T, x_{c, T}\right)=0
$$

so that $\bar{X}_{e}\left(t, T, x_{c, T}\right)=x_{c, T}$ for all $t \in[0, T]$. This implies that the set of critical points of $\widehat{\psi}(t, \cdot)$ is invariant through the flow $\bar{X}_{e}$ and is then included in $\tilde{\omega}$.

We finally check the condition $\inf _{\mathcal{O}} \widehat{\psi}(t, \cdot)=\widehat{\psi}(t)_{\mid \partial \mathcal{O}}$ for all $t \in[0, T]$ by contradiction. If this were wrong, there would exist $t \in[0, T]$ and $x_{t} \in \mathcal{O}$ such that $x_{t} \in \operatorname{Argmin} \widehat{\psi}(t, \cdot)$. Thus, $x_{t}$ would be a critical point, and as above, $\bar{X}_{e}\left(T, t, x_{t}\right)$ would belong to $\mathcal{O}$ and be a critical point of $\widehat{\psi}_{T}$. Following, $\widehat{\psi}\left(t, x_{t}\right)=\widehat{\psi}_{T}\left(\bar{X}_{e}\left(T, t, x_{t}\right)\right)$ would be larger than 0 due to the assumption on $\widehat{\psi}_{T}$. But from the boundary conditions, it follows that $\inf _{\mathcal{O}} \widehat{\psi}(t)$ cannot be strictly smaller than $\widehat{\psi}(t)_{\mid \partial \mathcal{O}}$, which is negative for all time $t \in[0, T)$.

Construction of $\tilde{\psi}$ in $[0, T] \times \mathbb{R}^{2}$. As $\mathcal{O}$ may share some boundary with $\Omega$, we need to explain that $\widehat{\psi}$ can be extended as a $C^{2}$ function in $[0, T] \times \mathbb{R}^{2}$. In order to do that, we extend $\widehat{\psi}(0, \cdot)$ as a $C^{2}$ function of $\mathbb{R}^{2}$, denoted $\widehat{\psi}_{0}$, and we solve

$$
\partial_{t} \widehat{\psi}+\overline{\mathbf{y}}_{e} \cdot \nabla \widehat{\psi}=0 \quad \text { in }(0, T) \times \mathbb{R}^{2}, \quad \widehat{\psi}(0, \cdot)=\widehat{\psi}_{0} \quad \text { in } \mathbb{R}^{2} .
$$

Of course, this is consistent with the definition of $\widehat{\psi}$ in (4.8), and the solution $\widehat{\psi}$ if (4.12) obviously is $C^{2}\left([0, T] \times \mathbb{R}^{2}\right)$ as $\widehat{\psi}_{0} \in C^{2}\left(\mathbb{R}^{2}\right)$.

One can then suitably choose $a>0$ and $b \in \mathbb{R}$ such that $\tilde{\psi}=a \widehat{\psi}+b$ satisfies $\tilde{\psi}(t, x) \in[0,1]$ for all $(t, x) \in \mathcal{O}_{T}$. Then $\tilde{\psi}$ satisfies all the required properties with $\overline{\mathbf{y}}=\overline{\mathbf{y}}_{e}$. This completes the proof of Lemma 4.1 .

\subsection{Proof of Theorem 4.2}

According to Section 3, the construction in Section 3.2 yields $\rho=\mathscr{F}_{1}\left(\widehat{\mathbf{u}}, \rho_{0}\right)$ solution of the control problem (1.19) for $\widehat{\mathbf{u}}$ satisfying (3.5). This condition is indeed satisfied for $\widehat{\mathbf{u}} \in \mathbf{X}_{s, \lambda}$ with $\|\widehat{\mathbf{u}}\| \mathbf{X}_{s, \lambda} \leq \varsigma$, see (3.18)-(3.20).

Theorem 3.6 immediately provides estimate (4.2), as $\lambda e^{6 \lambda(m+1)} \theta / 2 \leq 3 \varphi^{*} / 4$, see (2.13).

We then focus on the proof of the compactness property. According to the construction in Section 3.2, we introduce $\rho_{f, n}$ the solution of

$$
\begin{cases}\partial_{t} \rho_{f, n}+\left(\overline{\mathbf{y}}+\widehat{\mathbf{u}}_{n}\right) \cdot \nabla \rho_{f, n}=-\widehat{\mathbf{u}}_{n} \cdot \nabla \bar{\sigma} & \text { in } \Omega_{T}, \\ \rho_{f, n}(t, x)=0 & \text { for } t \in(0, T), x \in \partial \Omega, \text { with }\left(\overline{\mathbf{y}}(t, x)+\widehat{\mathbf{u}}_{n}(t, x)\right) \cdot \mathbf{n}(x)<0, \\ \rho_{f, n}(0)=\rho_{0} & \text { in } \Omega,\end{cases}
$$

$\rho_{b, n}$ the solution of

$$
\begin{cases}\partial_{t} \rho_{b, n}+\left(\overline{\mathbf{y}}+\widehat{\mathbf{u}}_{n}\right) \cdot \nabla \rho_{b, n}=-\widehat{\mathbf{u}}_{n} \cdot \nabla \bar{\sigma} & \text { in } \Omega_{T}, \\ \rho_{b, n}=0 & \text { for } t \in(0, T), x \in \partial \Omega, \text { with }\left(\overline{\mathbf{y}}(t, x)+\widehat{\mathbf{u}}_{n}(t, x)\right) \cdot \mathbf{n}(x)>0, \\ \rho_{b, n}(T)=0 & \text { in } \Omega,\end{cases}
$$

and $\chi_{n}$ the solution of

$$
\begin{cases}\partial_{t} \chi_{n}+\left(\overline{\mathbf{y}}+\widehat{\mathbf{u}}_{n}\right) \cdot \nabla \chi_{n}=0 & \text { in } \Omega_{T}, \\ \chi_{n}=1_{t \in\left(0, T_{0}\right)}(t) & \text { for } t \in(0, T), x \in \partial \Omega, \text { with }\left(\overline{\mathbf{y}}(t, x)+\widehat{\mathbf{u}}_{n}(t, x)\right) \cdot \mathbf{n}(x)<0, \\ \chi_{n}(0)=1 & \text { in } \Omega .\end{cases}
$$

Since $\widehat{\mathbf{u}}_{n}$ is a bounded sequence of $H^{1}\left(0, T ; \mathbf{L}^{2}(\Omega)\right) \cap L^{2}\left(0, T ; \mathbf{H}^{2}(\Omega)\right)$, which is compact in $L^{2}\left(0, T ; \mathbf{L}^{2}(\Omega)\right)$, up to a subsequence still denoted the same for simplicity, $\widehat{\mathbf{u}}_{n}$ converge to $\widehat{\mathbf{u}}$ weakly in $H^{1}\left(0, T ; \mathbf{L}^{2}(\Omega)\right) \cap L^{2}\left(0, T ; \mathbf{H}^{2}(\Omega)\right)$ and strongly in $L^{2}\left(0, T ; \mathbf{L}^{2}(\Omega)\right)$. Then [3, Theorem 4] applies and for all $q \in[1,+\infty)$ the sequence $\chi_{n}$ strongly converges towards $\chi$ in $L^{q}\left(\Omega_{T}\right)$ solution of (3.8).

Next, to pass to the limit in (4.13), we notice that $\sigma_{f, n}: \stackrel{\text { def }}{=} \bar{\sigma}+\rho_{f, n}$ solves

$$
\begin{cases}\partial_{t} \sigma_{f, n}+\left(\overline{\mathbf{y}}+\widehat{\mathbf{u}}_{n}\right) \cdot \nabla \sigma_{f, n}=0 & \text { in } \Omega_{T}, \\ \sigma_{f, n}(t, x)=0 & \text { for } t \in(0, T), x \in \partial \Omega, \text { with }\left(\overline{\mathbf{y}}(t, x)+\widehat{\mathbf{u}}_{n}(t, x)\right) \cdot \mathbf{n}(x)<0, \\ \sigma_{f, n}(0)=\bar{\sigma}_{0}+\rho_{0} & \text { in } \Omega .\end{cases}
$$

Thus, by applying again $\left[3\right.$, Theorem 4] we deduce that, for all $q \in[1,+\infty)$, the sequence $\sigma_{f, n}$ is strongly convergent in $L^{q}\left(\Omega_{T}\right)$ to the solution $\sigma_{f}$ of 


$$
\begin{cases}\partial_{t} \sigma_{f}+(\overline{\mathbf{y}}+\widehat{\mathbf{u}}) \cdot \nabla \sigma_{f}=0 & \text { in } \Omega_{T}, \\ \sigma_{f}(t, x)=0 & \text { for } t \in(0, T), x \in \partial \Omega, \text { with }(\overline{\mathbf{y}}(t, x)+\widehat{\mathbf{u}}(t, x)) \cdot \mathbf{n}(x)<0, \\ \sigma_{f}(0)=\bar{\sigma}_{0}+\rho_{0} & \text { in } \Omega .\end{cases}
$$

It follows that $\rho_{f, n}$ strongly converges in all $L^{q}\left(\Omega_{T}\right)$ for $q \in[1, \infty)$ to $\rho_{f}=\sigma_{f}-\bar{\sigma}$, which solves (3.6) by construction.

Of course, the same can be done to show that $\rho_{b, n}$ strongly converges in all $L^{q}\left(\Omega_{T}\right)$ for $q \in[1, \infty)$ to the solution $\rho_{b}$ of (3.7). Consequently, the sequence $\rho_{n}=\mathscr{F}_{1}\left(\widehat{\mathbf{u}}_{n}, \rho_{0}\right)$ converges to $\rho=\mathscr{F}_{1}\left(\widehat{\mathbf{u}}, \rho_{0}\right)$ in $L^{q}\left(\Omega_{T}\right)$ for all $q \in[1, \infty)$.

\subsection{Proof of Theorem 4.4}

Let $\rho_{0} \in L^{\infty}(\Omega), \mathbf{u}_{0} \in \mathbf{V}_{0}^{1}(\Omega)$ and $\widehat{\mathbf{u}} \in \mathbf{X}_{s, \lambda}$ with $\|\widehat{\mathbf{u}}\|_{\mathbf{X}_{s, \lambda}} \leq \varsigma$.

According to Theorem 4.2, $\rho=\mathscr{F}_{1}\left(\widehat{\mathbf{u}}, \rho_{0}\right)$ belongs to $Y_{s, \lambda}$ and is bounded in that space by (4.2). Thus, according to Theorem 4.3, for $\mathscr{F}$ to be well-defined, we have to check that $\mathbf{f}(\rho, \widehat{\mathbf{u}})$ given in (1.14) belongs to $\mathbf{F}_{s, \lambda}$, and we will get estimates on $\mathbf{u}=\mathscr{F}(\widehat{\mathbf{u}})$ from an estimate of $\mathbf{f}(\rho, \widehat{\mathbf{u}})$ in $\mathbf{F}_{s, \lambda}$ according to (4.3). We thus estimate $\mathbf{f}(\rho, \widehat{\mathbf{u}})$ in $\mathbf{F}_{s, \lambda}$ term by term from estimates on $\rho \in Y_{s, \lambda}$ and $\widehat{\mathbf{u}} \in \mathbf{X}_{s, \lambda}$.

We easily check

$$
\begin{aligned}
& \left\|\xi^{-2} e^{s \varphi} \rho\left(\partial_{t} \widehat{\mathbf{u}}+(\overline{\mathbf{y}}+\widehat{\mathbf{u}}) \cdot \nabla \widehat{\mathbf{u}}+\widehat{\mathbf{u}} \cdot \nabla \overline{\mathbf{y}}\right)\right\|_{L^{2}\left(\mathbf{L}^{2}\right)} \\
& \quad \leq\left\|e^{s \lambda e^{6 \lambda(m+1)} \theta / 2} \rho\right\|_{L^{\infty}}\left\|\xi^{-2} e^{s \varphi-s \lambda e^{6 \lambda(m+1)} \theta / 2}\left(\partial_{t} \widehat{\mathbf{u}}+((\overline{\mathbf{y}}+\widehat{\mathbf{u}}) \cdot \nabla) \widehat{\mathbf{u}}+\widehat{\mathbf{u}} \cdot \nabla \overline{\mathbf{y}}\right)\right\|_{L^{2}\left(\mathbf{L}^{2}\right)} \\
& \quad \leq C\|\rho\|_{Y_{s, \lambda}}\left\|e^{3 s \varphi^{*} / 4} \widehat{\mathbf{u}}\right\|_{L^{2}\left(\mathbf{H}^{2}\right) \cap H^{1}\left(\mathbf{L}^{2}\right)}\left\|\xi^{-2} e^{s \varphi-s \lambda e^{6 \lambda(m+1)} \theta / 2-3 s \varphi^{*} / 4}\right\|_{L^{\infty}},
\end{aligned}
$$

where we used that

$$
\|\overline{\mathbf{y}}+\widehat{\mathbf{u}}\|_{L^{2}\left(\mathbf{L}^{\infty}\right)} \leq C, \quad \text { and } \quad\left\|e^{3 s \varphi^{*} / 4} \nabla \widehat{\mathbf{u}}\right\|_{L^{\infty}\left(\mathbf{L}^{2}\right)} \leq C\left\|e^{3 s \varphi^{*} / 4} \widehat{\mathbf{u}}\right\|_{L^{2}\left(\mathbf{H}^{2}\right) \cap H^{1}\left(\mathbf{L}^{2}\right)} .
$$

According to (2.13), $s \varphi-s \lambda e^{6 \lambda(m+1)} \theta / 2-3 s \varphi^{*} / 4 \leq-s \varphi / 4$, and thus there exists some constant $C$ independent of $s$ and $\lambda$ such that

$$
\left\|\xi^{-2} e^{s \varphi-s \lambda e^{6 \lambda(m+1)} \theta / 2-3 s \varphi^{*} / 4}\right\|_{L^{\infty}} \leq C .
$$

Following,

$$
\left\|\xi^{-2} e^{s \varphi} \rho\left(\partial_{t} \widehat{\mathbf{u}}+(\overline{\mathbf{y}}+\widehat{\mathbf{u}}) \cdot \nabla \widehat{\mathbf{u}}\right)\right\|_{L^{2}\left(\mathbf{L}^{2}\right)} \leq C\|\rho\|_{Y_{s, \lambda}}\|\widehat{\mathbf{u}}\|_{\mathbf{X}_{s, \lambda}} .
$$

Next, we estimate $\bar{\sigma}(\widehat{\mathbf{u}} \cdot \nabla) \widehat{\mathbf{u}}$. Similarly as above, we write

$$
\left\|\xi^{-2} e^{s \varphi} \widehat{\sigma} \widehat{\mathbf{u}} \cdot \nabla \widehat{\mathbf{u}}\right\|_{L^{2}\left(\mathbf{L}^{2}\right)} \leq C\left\|e^{3 s \varphi^{*} / 4} \widehat{\mathbf{u}}\right\|_{L^{\infty}\left(\mathbf{L}^{4}\right)}\left\|e^{3 s \varphi^{*} / 4} \nabla \widehat{\mathbf{u}}\right\|_{L^{2}\left(\mathbf{L}^{4}\right)}\left\|\xi^{-2} e^{s \varphi-3 s \varphi^{*} / 2}\right\|_{L^{\infty}} \leq C\|\widehat{\mathbf{u}}\|_{\mathbf{X}_{s, \lambda}}^{2} .
$$

Last, we estimate $\rho\left(\partial_{t} \overline{\mathbf{y}}+(\overline{\mathbf{y}} \cdot \nabla) \overline{\mathbf{y}}\right)$ :

$$
\left\|\xi^{-2} e^{s \varphi} \rho\left(\partial_{t} \overline{\mathbf{y}}+(\overline{\mathbf{y}} \cdot \nabla) \overline{\mathbf{y}}\right)\right\|_{L^{2}\left(\mathbf{L}^{2}\right)} \leq C\left\|\xi^{-2} e^{s \varphi} \rho\right\|_{L^{2}} \leq C\|\rho\|_{Y_{s, \lambda}} .
$$

Putting estimates (4.18)-(4.20) together, we obtain:

$$
\|\mathbf{f}(\rho, \widehat{\mathbf{u}})\|_{\mathbf{F}_{s, \lambda}}=\left\|\xi^{-2} e^{s \varphi} \mathbf{f}(\rho, \widehat{\mathbf{u}})\right\|_{L^{2}\left(\mathbf{L}^{2}\right)} \leq C\left(\|\rho\|_{Y_{s, \lambda}}+\|\rho\|_{Y_{s, \lambda}}^{2}+\|\widehat{\mathbf{u}}\|_{\mathbf{X}_{s, \lambda}}^{2}\right) .
$$

Combined with estimates (4.2) and (4.3), this yields the well-posedness of the mapping $\mathscr{F}$ for $\widehat{\mathbf{u}} \in \mathbf{X}_{s, \lambda}$ with $\|\widehat{\mathbf{u}}\|_{\mathbf{X}_{s, \lambda}} \leq \varsigma$ and the estimate (4.4).

We now focus on the last part of Theorem 4.4. Let $\widehat{\mathbf{u}}_{n}$ is a sequence of $\mathbf{X}_{s, \lambda}$ with $\left\|\widehat{\mathbf{u}}_{n}\right\|_{\mathbf{X}_{s, \lambda}} \leq \varsigma$ which weakly converges to $\widehat{\mathbf{u}}$. Note that this weak convergence implies that $\|\widehat{\mathbf{u}}\|_{\mathbf{X}_{s, \lambda}} \leq \varsigma$, so that $\mathscr{F}(\widehat{\mathbf{u}})$ is well-defined.

Besides that, according to Theorem 4.2, the sequence $\rho_{n}=\mathscr{F}_{1}\left(\widehat{\mathbf{u}}_{n}, \rho_{0}\right)$ strongly converges in all $L^{q}\left(\Omega_{T}\right)$ with $q<\infty$ to $\rho=\mathscr{F}_{1}\left(\widehat{\mathbf{u}}, \rho_{0}\right)$ and the sequence $\rho_{n}$ is uniformly bounded in $Y_{s, \lambda}$.

We then have to check that $\mathbf{f}\left(\rho_{n}, \widehat{\mathbf{u}}_{n}\right)$ weakly converges in $\mathbf{F}_{s, \lambda}$ to $\mathbf{f}(\rho, \widehat{\mathbf{u}})$. But (4.21) shows that the sequence $\mathbf{f}\left(\rho_{n}, \widehat{\mathbf{u}}_{n}\right)$ is bounded in $\mathbf{F}_{s, \lambda}$, and thus we only need to prove that the sequence $\mathbf{f}\left(\rho_{n}, \widehat{\mathbf{u}}_{n}\right)$ weakly converges in $\mathcal{D}^{\prime}\left(\Omega_{T}\right)$ to $\mathbf{f}(\rho, \widehat{\mathbf{u}})$. To obtain this convergence result in $\mathcal{D}^{\prime}\left(\Omega_{T}\right)$, as $\rho_{n}$ strongly converges to $\rho$ in all $L^{q}\left(\Omega_{T}\right)$ with $q<\infty$ and $\widehat{\mathbf{u}}_{n}$ weakly converges to $\widehat{\mathbf{u}}$ in $H^{1}\left(0, T ; \mathbf{L}^{2}(\Omega)\right) \cap L^{2}\left(0, T ; \mathbf{H}^{2}(\Omega)\right)$, we only have to focus on the convergence of the 
term $\left(\bar{\sigma}+\rho_{n}\right) \widehat{\mathbf{u}}_{n} \cdot \nabla \widehat{\mathbf{u}}_{n}$. But, using the compactness of $H^{1}\left(0, T ; \mathbf{L}^{2}(\Omega)\right) \cap L^{2}\left(0, T ; \mathbf{H}^{2}(\Omega)\right)$ in $L^{4}\left(0, T ; \mathbf{L}^{4}(\Omega)\right)$, we have the convergences

$$
\begin{array}{cl}
\bar{\sigma}+\rho_{n} \underset{n \rightarrow \infty}{\longrightarrow} \bar{\sigma}+\rho & \text { strongly in } L^{q}\left(\Omega_{T}\right), q \in[1, \infty), \\
\widehat{\mathbf{u}}_{n} \underset{n \rightarrow \infty}{\longrightarrow} \widehat{\mathbf{u}} & \text { strongly in } L^{4}\left(0, T ; \mathbf{L}^{4}(\Omega)\right), \\
\nabla \widehat{\mathbf{u}}_{n} \underset{n \rightarrow \infty}{\longrightarrow} \nabla \widehat{\mathbf{u}} & \text { weakly in } L^{2}\left(0, T ; \mathbf{L}^{2}(\Omega)\right),
\end{array}
$$

so that, choosing $q=4$ for instance, we obtain the weak convergence of $\left(\bar{\sigma}+\rho_{n}\right) \widehat{\mathbf{u}}_{n} \cdot \nabla \widehat{\mathbf{u}}_{n}$ to $(\bar{\sigma}+\rho) \widehat{\mathbf{u}} \cdot \nabla \widehat{\mathbf{u}}$.

Following, $\mathbf{f}\left(\rho_{n}, \widehat{\mathbf{u}}_{n}\right)$ weakly converges in $\mathbf{F}_{s, \lambda}$ to $\mathbf{f}(\rho, \widehat{\mathbf{u}})$ and, since $\mathscr{F}_{2}: \mathbf{F}_{s, \lambda} \times \mathbf{V}_{0}^{1}(\Omega) \rightarrow \mathbf{X}_{s, \lambda}$ is a linear bounded operator, we obtain that $\mathbf{u}_{n}=\mathscr{F}\left(\widehat{\mathbf{u}}_{n}\right)=\mathscr{F}_{2}\left(\mathbf{f}\left(\rho_{n}, \widehat{\mathbf{u}}_{n}\right), \mathbf{u}_{0}\right)$ weakly converges to $\mathscr{F}_{2}\left(\mathbf{f}(\rho, \widehat{\mathbf{u}}), \mathbf{u}_{0}\right)=\mathscr{F}(\widehat{\mathbf{u}})=\mathbf{u}$ in $\mathbf{X}_{s, \lambda}$. Finally, as $\mathbf{X}_{s, \lambda}$ is compact in $L^{2}\left(0, T ; \mathbf{L}^{2}(\Omega)\right), \mathbf{u}_{n}$ strongly converges to $\mathbf{u}$ in $L^{2}\left(0, T ; \mathbf{L}^{2}(\Omega)\right)$.

\section{Conflict of interest statement}

None to declare.

\section{Acknowledgements}

The authors thank O. Glass, E. Zuazua and the anonymous referee for valuable comments on the preliminary version of this work.

\section{Appendix A. Proofs of Theorems 2.5 and 2.6}

For simplicity, we make the proofs of Theorems 2.5 and 2.6 for $v$ of equal to 1 . This can be done without loss of generality by replacing $\bar{\sigma}$ and $f$ by $\bar{\sigma} / v$ and $f / v$ if needed.

\section{A.1. Proof of Theorem 2.5}

Let $z$ be a smooth function on $[0, T] \times \overline{\mathcal{O}}$ satisfying $z=0$ on $(0, T) \times \partial \mathcal{O}$ and set

$$
f: \stackrel{\text { def }}{=}-\bar{\sigma} \partial_{t} z-\Delta z, \quad(t, x) \in(0, T) \times \mathcal{O} .
$$

Set then

$$
w=e^{-s \varphi} z \text {. }
$$

According to the definition of $\theta$ in (2.10), $w$ satisfies

$$
w(T, x)=0, \quad \nabla w(T, x)=0, \quad x \in \mathcal{O},
$$

in addition to the conditions $w(t, x)=0$ on $(0, T) \times \partial \mathcal{O}$.

Besides, with $f$ as in (A.1), $w$ satisfies

$$
e^{-s \varphi} f=e^{-s \varphi}\left(-\bar{\sigma} \partial_{t} z-\Delta z\right)=e^{-s \varphi}\left(-\bar{\sigma} \partial_{t}\left(e^{s \varphi} w\right)-\Delta\left(e^{s \varphi} w\right)\right)=P_{\varphi} w,
$$

where the operator $P_{\varphi}$ is given by

$$
P_{\varphi} w=-\bar{\sigma} \partial_{t} w-s \bar{\sigma} \partial_{t} \varphi w-\Delta w-2 s \nabla \varphi \cdot \nabla w-s^{2}|\nabla \varphi|^{2} w-s \Delta \varphi w .
$$

We now set $P_{1}, P_{2}$ and $R$ the operators:

$$
\begin{aligned}
& P_{1} w=-\bar{\sigma} \partial_{t} w-2 s \nabla \varphi \cdot \nabla w+2 s \lambda^{2}|\nabla \psi|^{2} \xi w, \\
& P_{2} w=-\Delta w-s \bar{\sigma} \partial_{t} \varphi w-s^{2}|\nabla \varphi|^{2} w, \\
& R w=s \lambda \Delta \psi \xi w-s \lambda^{2}|\nabla \psi|^{2} \xi w,
\end{aligned}
$$

so that 


$$
P_{\varphi}=P_{1}+P_{2}+R
$$

We then use that $P_{1} w+P_{2} w=f e^{-s \varphi}-R w$ and then

$$
\iint_{\mathcal{O}_{T}}\left|P_{1} w\right|^{2}+\iint_{\mathcal{O}_{T}}\left|P_{2} w\right|^{2}+2 \iint_{\mathcal{O}_{T}} P_{1} w P_{2} w=\iint_{\mathcal{O}_{T}}\left|f e^{-s \varphi}-R w\right|^{2} \leq 2 \iint_{\mathcal{O}_{T}}|f|^{2} e^{-2 s \varphi}+2 \iint_{\mathcal{O}_{T}}|R w|^{2} .
$$

The main part of the proof then consists in computing the scalar product of $P_{1} w$ with $P_{2} w$ and estimate it from below.

Computations. We write

$$
\iint_{\mathcal{O}_{T}} P_{1} w P_{2} w=\sum_{i, j=1}^{3} I_{i j}
$$

where $I_{i, j}$ is the scalar product of the $i$-th term of $P_{1} w$ with the $j$-th term of $P_{2} w$.

Computation of $I_{11}$.

$$
\begin{aligned}
I_{11} & =\iint_{\mathcal{O}_{T}} \bar{\sigma} \partial_{t} w \Delta w=-\iint_{\mathcal{O}_{T}} \bar{\sigma} \partial_{t}\left(\frac{|\nabla w|^{2}}{2}\right)-\iint_{\mathcal{O}_{T}} \partial_{t} w \nabla \bar{\sigma} \cdot \nabla w \\
& =\frac{1}{2} \int_{\mathcal{O}} \bar{\sigma}(0)|\nabla w(0)|^{2}+\frac{1}{2} \iint_{\mathcal{O}_{T}} \partial_{t} \bar{\sigma}|\nabla w|^{2}-\iint_{\mathcal{O}_{T}} \partial_{t} w \nabla \bar{\sigma} \cdot \nabla w .
\end{aligned}
$$

Computation of $I_{12}$.

$$
I_{12}=s \iint_{\mathcal{O}_{T}} \bar{\sigma}^{2} \partial_{t} w \partial_{t} \varphi w=-\frac{s}{2} \int_{\mathcal{O}} \bar{\sigma}^{2}(0) \partial_{t} \varphi(0)|w(0)|^{2}-\frac{s}{2} \iint_{\mathcal{O}_{T}} \bar{\sigma}^{2} \partial_{t t} \varphi|w|^{2}-s \iint_{\mathcal{O}_{T}} \bar{\sigma} \partial_{t} \bar{\sigma} \partial_{t} \varphi|w|^{2} .
$$

Computation of $I_{13}$.

$$
\begin{aligned}
I_{13} & =s^{2} \iint_{\mathcal{O}_{T}} \bar{\sigma} \partial_{t} w|\nabla \varphi|^{2} w \\
& =-\frac{s^{2}}{2} \int_{\mathcal{O}} \bar{\sigma}(0)|\nabla \varphi(0)|^{2}|w(0)|^{2}-\frac{s^{2}}{2} \iint_{\mathcal{O}_{T}} \bar{\sigma} \partial_{t}\left(|\nabla \varphi|^{2}\right)|w|^{2}-\frac{s^{2}}{2} \iint_{\mathcal{O}_{T}} \partial_{t} \bar{\sigma}|\nabla \varphi|^{2}|w|^{2} .
\end{aligned}
$$

Computation of $I_{21}$.

$$
\begin{aligned}
I_{21} & =2 s \iint_{\mathcal{O}_{T}} \nabla \varphi \cdot \nabla w \Delta w \\
& =2 s \int_{\Gamma_{T}} \partial_{\mathbf{n}} \varphi\left|\partial_{\mathbf{n}} w\right|^{2}-2 s \iint_{\mathcal{O}_{T}} \nabla(\nabla \varphi \cdot \nabla w) \cdot \nabla w \\
& =2 s \int_{\Gamma_{T}} \partial_{\mathbf{n}} \varphi\left|\partial_{\mathbf{n}} w\right|^{2}-2 s \iint_{\mathcal{O}_{T}} D^{2} \varphi(\nabla w, \nabla w)-s \iint_{\mathcal{O}_{T}} \nabla \varphi \cdot \nabla\left(|\nabla w|^{2}\right) \\
& =s \int_{\Gamma_{T}} \partial_{\mathbf{n}} \varphi\left|\partial_{\mathbf{n}} w\right|^{2}-2 s \iint_{\mathcal{O}_{T}} D^{2} \varphi(\nabla w, \nabla w)+s \iint_{\mathcal{O}_{T}} \Delta \varphi|\nabla w|^{2} .
\end{aligned}
$$

Computation of $I_{22}$. 


$$
\begin{aligned}
I_{22} & =2 s^{2} \iint_{\mathcal{O}_{T}} \bar{\sigma} \nabla \varphi \cdot \nabla w \partial_{t} \varphi w=-s^{2} \iint_{\mathcal{O}_{T}} \operatorname{div}\left(\bar{\sigma} \partial_{t} \varphi \nabla \varphi\right)|w|^{2} \\
& =-s^{2} \iint_{\mathcal{O}_{T}} \bar{\sigma} \operatorname{div}\left(\partial_{t} \varphi \nabla \varphi\right)|w|^{2}-s^{2} \iint_{\mathcal{O}_{T}} \nabla \bar{\sigma} \cdot \nabla \varphi \partial_{t} \varphi|w|^{2} .
\end{aligned}
$$

Computation of $I_{23}$.

$$
I_{23}=2 s^{3} \iint_{\mathcal{O}_{T}} \nabla \varphi \cdot \nabla w|\nabla \varphi|^{2} w=-s^{3} \iint_{\mathcal{O}_{T}} \operatorname{div}\left(|\nabla \varphi|^{2} \nabla \varphi\right)|w|^{2} .
$$

Computation of $I_{31}$.

$$
\begin{aligned}
I_{31} & =-2 s \lambda^{2} \iint_{\mathcal{O}_{T}}|\nabla \psi|^{2} \xi w \Delta w \\
& =2 s \lambda^{2} \iint_{\mathcal{O}_{T}}|\nabla \psi|^{2} \xi|\nabla w|^{2}+2 s \lambda^{2} \iint_{\mathcal{O}_{T}} \nabla\left(|\nabla \psi|^{2} \xi\right) w \cdot \nabla w .
\end{aligned}
$$

Computation of $I_{32}$.

$$
I_{32}=-2 s^{2} \lambda^{2} \iint_{\mathcal{O}_{T}} \bar{\sigma}|\nabla \psi|^{2} \xi \partial_{t} \varphi|w|^{2} .
$$

\section{Computation of $I_{33}$.}

$$
I_{33}=-2 s^{3} \lambda^{2} \iint_{\mathcal{O}_{T}}|\nabla \psi|^{2} \xi|\nabla \varphi|^{2}|w|^{2} .
$$

Combining the above computations (A.9)-(A.17), we obtain the following:

$$
\begin{aligned}
& \iint_{\mathcal{O}_{T}} P_{1} w P_{2} w \\
& =\frac{1}{2} \int_{\mathcal{O}} \bar{\sigma}(0)|\nabla w(0)|^{2}+\frac{1}{2} \int_{\mathcal{O}}|w(0)|^{2} \bar{\sigma}(0)\left(-s^{2}|\nabla \varphi(0)|^{2}-s \bar{\sigma}(0) \partial_{t} \varphi(0)\right) \\
& \quad-2 s \iint_{\mathcal{O}_{T}} D^{2} \varphi(\nabla w, \nabla w)+s \iint_{\mathcal{O}_{T}}\left(\Delta \varphi+2 \lambda^{2}|\nabla \psi|^{2} \xi\right)|\nabla w|^{2} \\
& \quad+\iint_{\mathcal{O}_{T}}|w|^{2}\left(s^{3}\left(-\operatorname{div}\left(|\nabla \varphi|^{2} \nabla \varphi\right)-2 \lambda^{2}|\nabla \psi|^{2} \xi|\nabla \varphi|^{2}\right)\right. \\
& \quad+s^{2} \bar{\sigma}\left(-\partial_{t}\left(|\nabla \varphi|^{2}\right)-\left(\Delta \varphi+2 \lambda^{2}|\nabla \psi|^{2} \xi\right) \partial_{t} \varphi\right) \\
& \left.\quad+s \bar{\sigma}^{2}\left(-\frac{1}{2} \partial_{t t} \varphi\right)\right) \\
& \quad+s \int_{\Gamma_{T}} \partial_{\mathbf{n}} \varphi\left|\partial_{\mathbf{n}} w\right|^{2}+I_{\mathcal{R}},
\end{aligned}
$$

where 


$$
\begin{aligned}
I_{\mathcal{R}}= & \frac{1}{2} \iint_{\mathcal{O}_{T}} \partial_{t} \bar{\sigma}|\nabla w|^{2}+2 s \lambda^{2} \iint_{\mathcal{O}_{T}} \nabla\left(|\nabla \psi|^{2} \xi\right) w \cdot \nabla w-s \iint_{\mathcal{O}_{T}} \bar{\sigma} \partial_{t} \bar{\sigma} \partial_{t} \varphi|w|^{2} \\
& -\frac{s^{2}}{2} \iint_{\mathcal{O}_{T}} \partial_{t} \bar{\sigma}|\nabla \varphi|^{2}|w|^{2}-s^{2} \iint_{\mathcal{O}_{T}} \nabla \bar{\sigma} \nabla \varphi \partial_{t} \varphi|w|^{2}-\iint_{\mathcal{O}_{T}} \partial_{t} w \nabla \bar{\sigma} \cdot \nabla w .
\end{aligned}
$$

Positivity. Our main goal now is to check that the coefficients in the above integrals are positive, except perhaps on the observation set $\omega_{T}$. At this step, we will strongly rely upon the choice of the weight function $\varphi$ in (2.11), and on the formula

$$
\partial_{t} \varphi=\frac{\partial_{t} \theta}{\theta} \varphi-\lambda \partial_{t} \psi \xi, \quad \partial_{t} \xi=\frac{\partial_{t} \theta}{\theta} \xi+\lambda \partial_{t} \psi \xi
$$

In the following, to simplify notations, we will denote by $C$ generic positive large constants that do not depend on $s$ or $\lambda$ and by $c$ generic positive small constants independent of $s$ and $\lambda$. The constants may change from line to line.

Positivity of the terms (A.18) at $t=0$. Explicit computations yield

$$
-\partial_{t} \varphi(0)=\frac{\mu}{T_{0}}\left(\lambda e^{6 \lambda(m+1)}-e^{\lambda \psi(0)}\right)+2 \lambda \partial_{t} \psi(0) e^{\lambda \psi(0)} \geq c s \lambda^{3} e^{\lambda(12 m+2)}
$$

whereas

$$
|\nabla \varphi(0)|^{2} \leq C \lambda^{2}|\xi(0)|^{2} \leq C \lambda^{2} e^{2 \lambda(6 m+1)}
$$

Thus, with (2.2), for some $\lambda_{1}>0$, taking $\lambda \geq \lambda_{1} \geq 1$,

$$
\inf _{\overline{\mathcal{O}}}\left\{-s^{2}|\nabla \varphi(0)|^{2}-s \bar{\sigma}(0) \partial_{t} \varphi(0)\right\} \geq c s^{2} \lambda^{3} e^{2 \lambda(6 m+1)},
$$

and, following,

$$
\frac{1}{2} \int_{\mathcal{O}} \bar{\sigma}(0)|w(0)|^{2}\left(-s^{2}|\nabla \varphi(0)|^{2}-s \bar{\sigma}(0) \partial_{t} \varphi(0)\right) \geq c s^{2} \lambda^{3} e^{2 \lambda(6 m+1)} \int_{\mathcal{O}}|w(0)|^{2} .
$$

Positivity of the terms (A.19) involving the gradient. For $\eta \in \mathbb{R}^{N}$, we have

$$
\begin{aligned}
& -2 s D^{2} \varphi(\eta, \eta)+s\left(\Delta \varphi+2 \lambda^{2}|\nabla \psi|^{2} \xi\right)|\eta|^{2} \\
& =2 s \lambda^{2} \xi|\nabla \psi \cdot \eta|^{2}+s \lambda^{2} \xi|\nabla \psi|^{2}|\eta|^{2}+2 s \lambda \xi D^{2} \psi(\eta, \eta)-s \lambda \xi \Delta \psi|\eta|^{2} .
\end{aligned}
$$

Using (2.8), we get the existence of $\lambda_{2}=\lambda_{2}\left(\alpha,\left\|D^{2} \psi\right\|_{\infty}\right) \geq \lambda_{1}$ such that for all $\lambda \geq \lambda_{2}$ and $\eta \in \mathbb{R}^{N}$,

$$
\forall(t, x) \in \mathcal{O}_{T} \backslash \tilde{\omega}_{T}, \quad-2 s D^{2} \varphi(\eta, \eta)+s\left(\Delta \varphi+2 \lambda^{2}|\nabla \psi|^{2} \xi\right)|\eta|^{2} \geq c s \lambda^{2}|\eta|^{2} \xi,
$$

whereas there exists a positive constant $C=C\left(\alpha,\left\|D^{2} \psi\right\|_{\infty}\right)$ such that

$$
\forall \eta \in \mathbb{R}^{N}, \forall(t, x) \in \tilde{\omega}_{T}, \quad-2 s D^{2} \varphi(\eta, \eta)+s\left(\Delta \varphi+2 \lambda^{2}|\nabla \psi|^{2} \xi\right)|\eta|^{2} \geq c s \lambda^{2} \xi|\eta|^{2}-C s \lambda^{2} \xi|\eta|^{2} .
$$

Hence we obtain, for all $\lambda \geq \lambda_{1}$,

$$
-2 s \iint_{\mathcal{O}_{T}} D^{2} \varphi(\nabla w, \nabla w)+s \iint_{\mathcal{O}_{T}}\left(\Delta \varphi+2 \lambda^{2}|\nabla \psi|^{2} \xi\right)|\nabla w|^{2} \geq c s \lambda^{2} \iint_{\mathcal{O}_{T}} \xi|\nabla w|^{2}-C s \lambda^{2} \iint_{\tilde{\omega}_{T}} \xi|\nabla w|^{2} .
$$

Positivity of the terms (A.20) involving $w$ with scale $s^{3}$. Using $\nabla \varphi=-\lambda \nabla \psi \xi$, we have

$$
\begin{aligned}
& -\operatorname{div}\left(|\nabla \varphi|^{2} \nabla \varphi\right)=3 \lambda^{4}|\nabla \psi|^{4} \xi^{3}+\lambda^{3} \xi^{3} \operatorname{div}\left(|\nabla \psi|^{2} \nabla \psi\right), \\
& \lambda^{2}|\nabla \psi|^{2} \xi|\nabla \varphi|^{2}=\lambda^{4}|\nabla \psi|^{4} \xi^{3} .
\end{aligned}
$$

Hence 


$$
-\operatorname{div}\left(|\nabla \varphi|^{2} \nabla \varphi\right)-2 \lambda^{2}|\nabla \psi|^{2} \xi|\nabla \varphi|^{2}=\lambda^{4}|\nabla \psi|^{4} \xi^{3}+\lambda^{3} \xi^{3} \operatorname{div}\left(|\nabla \psi|^{2} \nabla \psi\right) .
$$

Using (2.8), we thus get the existence of $\lambda_{3}=\lambda_{3}\left(\alpha,\left\|D^{2} \psi\right\|_{\infty}\right) \geq \lambda_{2}$ such that for $\lambda \geq \lambda_{3}$,

$$
\forall(t, x) \in \mathcal{O}_{T} \backslash \omega_{T}, \quad-\operatorname{div}\left(|\nabla \varphi|^{2} \nabla \varphi\right)-2 \lambda^{2}|\nabla \psi|^{2} \xi|\nabla \varphi|^{2} \geq c \lambda^{4} \xi^{3},
$$

whereas there exists a positive constant $C=C\left(\alpha,\left\|D^{2} \psi\right\|_{\infty}\right)$ such that

$$
\forall(t, x) \in \tilde{\omega}_{T}, \quad-\operatorname{div}\left(|\nabla \varphi|^{2} \nabla \varphi\right)-2 \lambda^{2}|\nabla \psi|^{2} \xi|\nabla \varphi|^{2} \geq c \lambda^{4} \xi^{3}-C \lambda^{4} \xi^{3} .
$$

We thus obtain, for all $\lambda \geq \lambda_{3}$,

$$
s^{3} \iint_{\mathcal{O}_{T}}|w|^{2}\left(-\operatorname{div}\left(|\nabla \varphi|^{2} \nabla \varphi\right)-2 \lambda^{2}|\nabla \psi|^{2} \xi|\nabla \varphi|^{2}\right) \geq c s^{3} \lambda^{4} \iint_{\mathcal{O}_{T}} \xi^{3}|w|^{2}-C s^{3} \lambda^{4} \iint_{\tilde{\omega}_{T}} \xi^{3}|w|^{2} .
$$

Terms (A.21) involving $w$ in the scale $s^{2}$. We have to estimate

$$
-\partial_{t}\left(|\nabla \varphi|^{2}\right)-\left(\Delta \varphi+2 \lambda^{2}|\nabla \psi|^{2} \xi\right) \partial_{t} \varphi .
$$

Explicit computations yield:

$$
\begin{aligned}
-\partial_{t}\left(|\nabla \varphi|^{2}\right)-\left(\Delta \varphi+2 \lambda^{2}|\nabla \psi|^{2} \xi\right) \partial_{t} \varphi & \\
= & -\lambda^{3} \xi^{2} \partial_{t} \psi|\nabla \psi|^{2}-2 \lambda^{2} \xi^{2} \nabla \psi \cdot \nabla \partial_{t} \psi-\lambda^{2} \xi^{2} \partial_{t} \psi \Delta \psi \\
& +\frac{\partial_{t} \theta}{\theta}\left(-\lambda^{2} \xi \varphi|\nabla \psi|^{2}+\lambda \xi \Delta \psi \varphi-2 \lambda^{2} \xi^{2}|\nabla \psi|^{2}\right) .
\end{aligned}
$$

Before going further, let us remark that, using $\xi \geq 1$, there exists a positive constant $C$, only depending on the $C^{2}$-norm of $\psi$ such that for all $\lambda \geq 1$, for all $(t, x) \in(0, T) \times \mathcal{O}$,

$$
\left.\left|-\lambda^{3} \xi^{2} \partial_{t} \psi\right| \nabla \psi\right|^{2}-2 \lambda^{2} \xi^{2} \nabla \psi \cdot \nabla \partial_{t} \psi-\lambda^{2} \xi^{2} \partial_{t} \psi \Delta \psi-2 \lambda^{2} \xi^{2}|\nabla \psi|^{2} \mid \leq C \lambda^{3} \xi^{3} .
$$

This estimate is sufficient to handle the terms in (A.35).

We will then focus on the terms in (A.36). First remark that on $\left(T_{0}, T-2 T_{1}\right), \partial_{t} \theta \equiv 0$, so the term in (A.36) simply vanishes.

On $\left(T-2 T_{1}, T\right)$, we use the fact that there exists a constant $C>0$ such that

$$
\forall t \in\left(T-2 T_{1}, T\right), \quad\left|\partial_{t} \theta\right| \leq C \theta^{2} .
$$

Hence there exists $C=C\left(\|\nabla \psi\|_{\infty},\|\Delta \psi\|_{\infty}\right)$ such that for all $(t, x) \in\left(T-2 T_{1}, T\right) \times \mathcal{O}$,

$$
\left|\frac{\partial_{t} \theta}{\theta}\left(-\lambda^{2} \xi \varphi|\nabla \psi|^{2}+\lambda \xi \Delta \psi \varphi-2 \lambda^{2} \xi^{2}|\nabla \psi|^{2}\right)\right| \leq C \lambda^{2} \theta \xi \varphi \leq C \lambda^{3} \xi^{3},
$$

where for the last inequality we have used $|\theta \varphi| \leq \lambda \xi^{2}$, which is a consequence of (2.13).

On $\left(0, T_{0}\right)$, we are going to use that $\partial_{t} \theta \leq 0$ and $\theta \in[1,2]$ and thus the term in (A.36) has the good sign outside $\tilde{\omega}_{T}$. Indeed, using (2.8), we can find $\lambda_{4}=\lambda_{4}\left(\alpha,\|\Delta \psi\|_{\infty}\right) \geq \lambda_{3}$ such that for all $\lambda \geq \lambda_{4}$, for all $(t, x) \in\left(0, T_{0}\right) \times \mathcal{O}$ such that $(t, x) \notin \tilde{\omega}_{T}$,

$$
-\left(-\lambda^{2} \xi \varphi|\nabla \psi|^{2}+\lambda \xi \Delta \psi \varphi-2 \lambda^{2} \xi^{2}|\nabla \psi|^{2}\right) \geq c \lambda^{2} \xi \varphi
$$

whereas it is bounded by $C \lambda^{2} \xi \varphi$ everywhere in $\mathcal{O}_{T}$. We thus derive, for all $\lambda \geq \lambda_{4}$,

$$
\begin{aligned}
& s^{2} \iint_{\mathcal{O}_{T}}|w|^{2} \bar{\sigma}\left(-\partial_{t}\left(|\nabla \varphi|^{2}\right)-\left(\Delta \varphi+2 \lambda^{2}|\nabla \psi|^{2} \xi\right) \partial_{t} \varphi\right) \\
& \geq c s^{2} \lambda^{2} \int_{0}^{T_{0}} \int_{\mathcal{O}}\left|\partial_{t} \theta\right| \xi \varphi|w|^{2}-C s^{2} \lambda^{3} \iint_{\mathcal{O}_{T}} \xi^{3}|w|^{2}-C s^{2} \lambda^{2} \iint_{\tilde{\omega}_{T} \cap\left\{t \in\left(0, T_{0}\right)\right\}}\left|\partial_{t} \theta\right| \xi \varphi|w|^{2} .
\end{aligned}
$$


Term (A.22) involving $w$ in the scale $s$. We have to estimate $-\partial_{t t} \varphi$.

$$
\partial_{t t} \varphi=\frac{\partial_{t t} \theta}{\theta} \varphi-2 \lambda \frac{\partial_{t} \theta}{\theta} \partial_{t} \psi \xi-\lambda \partial_{t t} \psi \xi-\lambda^{2}\left(\partial_{t} \psi\right)^{2} \xi
$$

Let us first remark that we immediately have

$$
\left|-\lambda \partial_{t t} \psi \xi-\lambda^{2}\left(\partial_{t} \psi\right)^{2} \xi\right| \leq C \lambda^{2} \xi^{3} .
$$

For $t \in\left(0, T_{0}\right)$, we further have

$$
\forall t \in\left(0, T_{0}\right), \quad\left|\partial_{t t} \theta\right| \leq C s^{2} \lambda^{4} e^{\lambda(12 m-8)}, \quad\left|\partial_{t} \theta\right| \leq C s \lambda^{2} e^{\lambda(6 m-4)},
$$

so that, on $\left(0, T_{0}\right)$

$$
\left|\partial_{t t} \varphi\right| \leq C s^{2} \lambda^{5} e^{\lambda(12 m-8)} e^{6 \lambda(m+1)}+C s \lambda^{3} e^{\lambda(6 m-4)} \xi+C \lambda^{2} \xi^{3} \leq C s^{2} \lambda^{2} \xi^{3} .
$$

For $t \in\left(T-2 T_{1}, T\right)$, we have

$$
\forall t \in\left(T-2 T_{1}, T\right), \quad\left|\partial_{t t} \theta\right| \leq C \theta^{3} \quad \text { and } \quad\left|\partial_{t} \theta\right| \leq C \theta^{2} .
$$

Hence, using (2.13) and $\theta \varphi \leq \lambda \xi^{2}$, for some positive constant $C=C\left(\left\|\partial_{t} \psi\right\|_{\infty}\right)$,

$$
\forall(t, x) \in\left(T-2 T_{1}, T\right) \times \mathcal{O}, \quad\left|\partial_{t t} \varphi\right| \leq C \theta^{2} \varphi+C \lambda \theta \xi+C \lambda^{2} \xi^{3} \leq C \lambda^{2} \xi^{3} .
$$

Combining all these estimates, we get

$$
s \iint_{\mathcal{O}_{T}} \bar{\sigma}^{2}|w|^{2}\left(-\frac{1}{2} \partial_{t t} \varphi\right) \geq-C s^{3} \lambda^{2} \iint_{\mathcal{O}_{T}} \xi^{3}|w|^{2} .
$$

Positivity of the terms (A.20)-(A.22) involving $w$. Here we combine the estimates in (A.34), (A.38), (A.40) in order to derive suitable estimates for the sum of the terms in (A.20)-(A.22). To simplify notations, let us set $I_{w}$ the sum of the terms in (A.20)-(A.22):

$$
\begin{aligned}
I_{w}: \stackrel{\text { def }}{=} & \iint_{\mathcal{O}_{T}}|w|^{2}\left(s^{3}\left(-\operatorname{div}\left(|\nabla \varphi|^{2} \nabla \varphi\right)-2 \lambda^{2}|\nabla \psi|^{2} \xi|\nabla \varphi|^{2}\right)\right. \\
& \left.+s^{2} \bar{\sigma}\left(-\partial_{t}\left(|\nabla \varphi|^{2}\right)-\left(\Delta \varphi+2 \lambda^{2}|\nabla \psi|^{2} \xi\right) \partial_{t} \varphi\right)+s \bar{\sigma}^{2}\left(-\frac{1}{2} \partial_{t t} \varphi\right)\right) .
\end{aligned}
$$

Putting together (A.34), (A.38), (A.40), we deduce that there exist $s_{1} \geq 1$ and $\lambda_{5} \geq \lambda_{4}$ such that for $s \geq s_{1}$ and $\lambda \geq \lambda_{5}$,

$$
I_{w} \geq c s^{3} \lambda^{4} \iint_{\mathcal{O}_{T}} \xi^{3}|w|^{2}+c s^{2} \lambda^{2} \int_{0}^{T_{0}} \int_{\mathcal{O}}\left|\partial_{t} \theta\right| \xi \varphi|w|^{2}-C s^{3} \lambda^{4} \iint_{\tilde{\omega}_{T}} \xi^{3}|w|^{2}-C s^{2} \lambda^{2} \iint_{\tilde{\omega}_{T} \cap\left\{t \in\left(0, T_{0}\right)\right\}}\left|\partial_{t} \theta\right| \xi \varphi|w|^{2} .
$$

Positivity of the boundary terms (A.23). Here, we only have to remark that $\partial_{\mathbf{n}} \varphi \geq 0$ since $\partial_{\mathbf{n}} \psi \leq 0$ by construction, see (2.7).

A bound on $I_{\mathcal{R}}$ in (A.24). We also provide an upper bound on $I_{\mathcal{R}}$.

First, we shall of course use the immediate estimate

$$
\frac{1}{2} \iint_{\mathcal{O}_{T}} \partial_{t} \bar{\sigma}|\nabla w|^{2} \leq C \iint_{\mathcal{O}_{T}}|\nabla w|^{2} .
$$

Using $\nabla\left(|\nabla \psi|^{2} \xi\right) \leq C \lambda \xi$, one easily checks that

$$
\left|2 s \lambda^{2} \iint_{\mathcal{O}_{T}} \nabla\left(|\nabla \psi|^{2} \xi\right) w \cdot \nabla w\right| \leq C s^{2} \lambda^{4} \iint_{\mathcal{O}_{T}} \xi^{3}|w|^{2}+C \lambda^{2} \iint_{\mathcal{O}_{T}} \xi|\nabla w|^{2} .
$$


Using (A.25), we have

$$
\left|\partial_{t} \varphi\right| \leq \begin{cases}s \lambda^{2} e^{\lambda(6 m-4)} \lambda e^{6 \lambda(m+1)}+C \lambda \xi & \text { on }\left(0, T_{0}\right) \\ C \lambda \xi & \text { on }\left(T_{0}, T-2 T_{1}\right) \\ \theta \lambda e^{6 \lambda(m+1)}+C \lambda \xi & \text { on }\left(T-2 T_{1}, T\right)\end{cases}
$$

so that $\left|\partial_{t} \varphi\right| \leq C s \lambda \xi^{3}$ everywhere. Hence

$$
\left.\left.\left|s \iint_{\mathcal{O}_{T}} \bar{\sigma} \partial_{t} \bar{\sigma} \partial_{t} \varphi\right| w\right|^{2}\left|\leq C s^{2} \lambda \iint_{\mathcal{O}_{T}} \xi^{3}\right| w\right|^{2}
$$

Moreover, using $|\nabla \varphi| \leq C \lambda \xi$, (A.25) and $\theta \varphi \leq \lambda \xi^{2}$ we also obtain

$$
\begin{aligned}
\left.\left|\frac{s^{2}}{2} \iint_{\mathcal{O}_{T}} \partial_{t} \bar{\sigma}\right| \nabla \varphi\right|^{2}|w|^{2} \mid & \leq C s^{2} \lambda^{2} \iint_{\mathcal{O}_{T}} \xi^{2}|w|^{2}, \\
\left.\left|s^{2} \iint_{\mathcal{O}_{T}} \nabla \bar{\sigma} \nabla \varphi \partial_{t} \varphi\right| w\right|^{2} \mid & \leq C s^{2} \lambda \int_{0}^{T_{0}} \int_{\mathcal{O}} \xi \varphi\left|\partial_{t} \theta\right||w|^{2}+C s^{2} \lambda^{2} \iint_{\mathcal{O}_{T}} \xi^{3}|w|^{2} .
\end{aligned}
$$

Finally, we also have

$$
\left|\iint_{\mathcal{O}_{T}} \partial_{t} w \nabla \bar{\sigma} \cdot \nabla w\right| \leq C \frac{1}{s \lambda} \iint_{\mathcal{O}_{T}} \frac{1}{\xi}\left|\partial_{t} w\right|^{2}+C s \lambda \iint_{\mathcal{O}_{T}} \xi|\nabla w|^{2},
$$

and combining all the above estimates,

$$
\left|I_{\mathcal{R}}\right| \leq \frac{C}{s \lambda} \iint_{\mathcal{O}_{T}} \frac{1}{\xi}\left|\partial_{t} w\right|^{2}+C s \lambda \iint_{\mathcal{O}_{T}} \xi|\nabla w|^{2}+C s^{2} \lambda \int_{0}^{T_{0}} \int_{\mathcal{O}} \xi\left|\partial_{t} \theta\right| \varphi|w|^{2}+C s^{2} \lambda^{4} \iint_{\mathcal{O}_{T}} \xi^{3}|w|^{2} .
$$

A lower bound for the cross-product $\iint P_{1} w P_{2} w$. This step simply consists in putting together all the above estimates: for all $s \geq s_{1}$ and $\lambda \geq \lambda_{5}$,

$$
\begin{aligned}
2 \iint_{\mathcal{O}_{T}} P_{1} w P_{2} w \geq & \int_{\mathcal{O}}|\nabla w(0)|^{2}+c s^{2} \lambda^{3} e^{12 \lambda m+2} \int_{\mathcal{O}}|w(0)|^{2} \\
& +c s \lambda^{2} \iint_{\mathcal{O}_{T}} \xi|\nabla w|^{2}-C s \lambda^{2} \iint_{\tilde{\omega}_{T}} \xi|\nabla w|^{2} \\
& +c s^{3} \lambda^{4} \iint_{\mathcal{O}_{T}} \xi^{3}|w|^{2}+c s^{2} \lambda^{2} \int_{0}^{T_{0}} \int_{\mathcal{O}}\left|\partial_{t} \theta\right| \xi \varphi|w|^{2} \\
& -C s^{3} \lambda^{4} \iint_{\tilde{\omega}_{T}} \xi^{3}|w|^{2}-C s^{2} \lambda^{2} \int_{\tilde{\omega}_{T} \cap\left\{t \in\left(0, T_{0}\right)\right\}}\left|\partial_{t} \theta\right| \xi \varphi|w|^{2}-\left|I_{\mathcal{R}}\right| .
\end{aligned}
$$

Thus, using (A.46), for some $s_{2} \geq s_{1}$ and $\lambda_{6} \geq \lambda_{5}$, for all $s \geq s_{2}$ and $\lambda \geq \lambda_{6}$

$$
\begin{aligned}
2 \iint_{\mathcal{O}_{T}} P_{1} w P_{2} w \geq & \int_{\mathcal{O}}|\nabla w(0)|^{2}+c s^{2} \lambda^{3} e^{12 \lambda m+2} \int_{\mathcal{O}}|w(0)|^{2}+c s \lambda^{2} \iint_{\mathcal{O}_{T}} \xi|\nabla w|^{2}-C s \lambda^{2} \iint_{\tilde{\omega}_{T}} \xi|\nabla w|^{2} \\
& +c s^{3} \lambda^{4} \iint_{\mathcal{O}_{T}} \xi^{3}|w|^{2}+c s^{2} \lambda^{2} \int_{0}^{T_{0}} \int_{\mathcal{O}}\left|\partial_{t} \theta\right| \xi \varphi|w|^{2}-C s^{3} \lambda^{4} \iint_{\tilde{\omega}_{T}} \xi^{3}|w|^{2}
\end{aligned}
$$




$$
-C s^{2} \lambda^{2} \int_{\tilde{\omega}_{T} \cap\left\{t \in\left(0, T_{0}\right)\right\}}\left|\partial_{t} \theta\right| \xi \varphi|w|^{2}-\frac{C}{s \lambda} \int_{0}^{T} \int_{\mathcal{O}} \frac{1}{\xi}\left|\partial_{t} w\right|^{2} .
$$

Conclusion. We first derive a Carleman estimate on $w$ with observations on the gradient, and then explains how to remove this term using a suitable multiplier.

A Carleman estimate on $w$ with observations on the gradient. According to estimates (A.8) and (A.47), for all $s \geq s_{2}$ and $\lambda \geq \lambda_{6}$,

$$
\begin{aligned}
& \iint_{\mathcal{O}_{T}}\left(\left|P_{1} w\right|^{2}+\left|P_{2} w\right|^{2}\right)+c \int_{\mathcal{O}}|\nabla w(0)|^{2}+c s^{2} \lambda^{3} e^{12 \lambda m+2} \int_{\mathcal{O}}|w(0)|^{2} \\
& \quad+c s \lambda^{2} \iint_{\mathcal{O}_{T}} \xi|\nabla w|^{2}+c s^{3} \lambda^{4} \iint_{\mathcal{O}_{T}} \xi^{3}|w|^{2}+c s^{2} \lambda^{2} \iint_{0}^{T_{0}}\left|\partial_{t} \theta\right| \varphi \xi|w|^{2} \\
& \leq C \iint_{\mathcal{O}_{T}}|f|^{2} e^{-2 s \varphi}+C \iint_{\mathcal{O}_{T}}|R w|^{2}+C s \lambda^{2} \iint_{\tilde{\omega}_{T}} \xi|\nabla w|^{2} \\
& \quad+C s^{3} \lambda^{4} \iint_{\tilde{\omega}_{T}} \xi^{3}|w|^{2}+C s^{2} \lambda^{2} \iint_{\tilde{\omega}_{T} \cap\left\{t \in\left(0, T_{0}\right)\right\}}\left|\partial_{t} \theta\right| \xi \varphi|w|^{2}+\frac{C}{s \lambda} \iint_{\mathcal{O}_{T}} \frac{1}{\xi}\left|\partial_{t} w\right|^{2} .
\end{aligned}
$$

To handle the term $\|R w\|_{L^{2}}^{2}$, we recall that $R w$ is given by (A.7), hence

$$
\iint_{\mathcal{O}_{T}}|R w|^{2} \leq C s^{2} \lambda^{4} \iint_{\mathcal{O}_{T}} \xi^{3}|w|^{2}
$$

where $C=C\left(\|\nabla \psi\|_{\infty},\|\Delta \psi\|_{\infty}\right)$ is a positive constant.

Also note that

$$
\frac{1}{s \lambda} \iint_{\mathcal{O}_{T}} \frac{1}{\xi}\left|\partial_{t} w\right|^{2} \leq \frac{C}{s \lambda} \iint_{\mathcal{O}_{T}}\left|P_{1} w\right|^{2}+C s \lambda \iint_{\mathcal{O}_{T}} \xi|\nabla w|^{2}+C s \lambda^{3} \iint_{\mathcal{O}_{T}} \xi^{3}|w|^{2} .
$$

In particular, for some $s_{3} \geq s_{2}$, for all $s \geq s_{3}$ and $\lambda \geq \lambda_{6}$,

$$
\begin{aligned}
& \iint_{\mathcal{O}_{T}}\left(\left|P_{1} w\right|^{2}+\left|P_{2} w\right|^{2}\right)+c \int_{\mathcal{O}}|\nabla w(0)|^{2}+c s^{2} \lambda^{3} e^{12 \lambda m+2} \int_{\mathcal{O}}|w(0)|^{2} \\
& \quad+c s \lambda^{2} \iint_{\mathcal{O}_{T}} \xi|\nabla w|^{2}+c s^{3} \lambda^{4} \iint_{\mathcal{O}_{T}} \xi^{3}|w|^{2}+c s^{2} \lambda^{2} \int_{0}^{T_{0}} \int_{\mathcal{O}}\left|\partial_{t} \theta\right| \xi \varphi|w|^{2} \\
& \quad \leq C \iint_{\mathcal{O}_{T}}|f|^{2} e^{-2 s \varphi}+C s \lambda^{2} \iint_{\tilde{\omega}_{T}} \xi|\nabla w|^{2}+C s^{3} \lambda^{4} \iint_{\tilde{\omega}_{T}} \xi^{3}|w|^{2}+C s^{2} \lambda^{2} \iint_{\tilde{\omega}_{T} \cap\left\{t \in\left(0, T_{0}\right)\right\}}\left|\partial_{t} \theta\right| \xi \varphi|w|^{2} .
\end{aligned}
$$

In (A.48), the observation is done on $\tilde{\omega}_{T}$ and concerns both $w$ and $\nabla w$. Below, we shall explain that this observation can be done only on $w$ provided we take an observation set slightly larger.

A Carleman estimate on $w$ without observations on the gradient. Recall that $\tilde{\omega}_{T} \Subset \widehat{\omega_{T}}$, then there exists a nonnegative smooth function $\eta=\eta(t, x)$ taking value in $[0,1]$ such that $\eta=1$ on $\tilde{\omega}_{T}$, and $\eta=0$ in $(0, T) \times \mathcal{O} \backslash \widehat{\omega_{T}}$. We then compute the scalar product of $P_{2} w$ and $\eta s \lambda^{2} \xi w$ :

$$
\iint_{\mathcal{O}_{T}} P_{2} w\left(\eta s \lambda^{2} \xi w\right)=s \lambda^{2} \iint_{\mathcal{O}_{T}} \eta \xi|\nabla w|^{2}-\frac{s \lambda^{2}}{2} \iint_{\mathcal{O}_{T}} \Delta(\eta \xi)|w|^{2}
$$




$$
-s^{2} \lambda^{2} \iint_{\mathcal{O}_{T}} \eta \bar{\sigma} \partial_{t} \varphi \xi|w|^{2}-s^{3} \lambda^{2} \iint_{\mathcal{O}_{T}} \eta|\nabla \varphi|^{2} \xi|w|^{2} .
$$

In particular, using (A.25) and (2.13),

$$
\begin{aligned}
& s \lambda^{2} \iint_{\mathcal{O}_{T}} \eta \xi|\nabla w|^{2}+c s^{2} \lambda^{2} \int_{0}^{T_{0}} \int_{\mathcal{O}} \bar{\sigma} \eta\left|\partial_{t} \theta\right| \xi \varphi|w|^{2} \\
& \leq \iint_{\mathcal{O}_{T}} P_{2} w\left(\eta s \lambda^{2} \xi w\right)+s \lambda^{2} \int_{\mathcal{O}_{T}}|\Delta(\eta \xi)||w|^{2}+s^{2} \lambda^{3} e^{6 \lambda(m+1)} \int_{T-2 T_{1}}^{T} \int_{\mathcal{O}} \eta \bar{\sigma}\left|\partial_{t} \theta\right| \xi|w|^{2} \\
& \quad+s^{2} \lambda^{3} \iint_{\mathcal{O}_{T}} \eta \bar{\sigma}\left|\partial_{t} \psi\right| \xi^{2}|w|^{2}+s^{3} \lambda^{4} \iint_{\mathcal{O}_{T}} \eta|\nabla \psi|^{2} \xi^{3}|w|^{2} .
\end{aligned}
$$

Of course, this implies that

$$
\begin{aligned}
& s \lambda^{2} \iint_{\tilde{\omega}_{T}} \xi|\nabla w|^{2}+s^{2} \lambda^{2} \iint_{\tilde{\omega}_{T} \cap\left\{t \in\left(0, T_{0}\right)\right\}} \bar{\sigma} \eta\left|\partial_{t} \theta\right| \xi \varphi|w|^{2} \\
& \leq \frac{1}{\sqrt{s}} \iint_{\mathcal{O}_{T}}\left|P_{2} w\right|^{2}+C s^{5 / 2} \lambda^{4} \iint_{\mathcal{O}_{T}} \eta^{2} \xi^{2}|w|^{2}+2 C s \lambda^{2} \iint_{\mathcal{O}_{T}}|\Delta(\eta \xi)||w|^{2} \\
& \quad+2 C s^{2} \lambda^{3} e^{6 \lambda(m+1)} \int_{T-2 T_{1}}^{T} \int_{\mathcal{O}} \eta\left|\partial_{t} \theta\right| \xi|w|^{2}+2 C s^{2} \lambda^{3} \iint_{\mathcal{O}_{T}} \eta\left|\partial_{t} \psi\right| \xi^{2}|w|^{2}+2 C s^{3} \lambda^{4} \iint_{\widehat{\omega_{T}}}|\nabla \psi|^{2} \xi^{3}|w|^{2} .
\end{aligned}
$$

But there exists a constant $C=C\left(\|\eta\|_{L^{\infty}\left(C^{2}\right)},\|\nabla \psi\|_{\infty},\|\Delta \psi\|_{\infty},\left\|\partial_{t} \psi\right\|_{\infty}\right)$ such that

$$
|\Delta(\eta \xi)| \leq C \lambda^{2} \xi^{2}, \quad \sup _{\left[T-2 T_{1}, T\right)}\left\{\frac{\left|\partial_{t} \theta\right|}{\theta^{2}}\right\} \leq C
$$

hence, using the fact that $\eta$ is supported on $\widehat{\omega_{T}}$,

$$
s^{5 / 2} \lambda^{4} \iint_{\mathcal{O}_{T}} \eta^{2} \xi^{2}|w|^{2}+s \lambda^{2} \iint_{\mathcal{O}_{T}}|\Delta(\eta \xi)||w|^{2}+s^{2} \lambda^{3} \iint_{\mathcal{O}_{T}} \eta\left|\partial_{t} \psi\right| \xi^{2}|w|^{2} \leq C s^{3} \lambda^{4} \iint_{\widehat{\omega}_{T}} \xi^{3}|w|^{2},
$$

whereas

$$
s^{2} \lambda^{3} e^{6 \lambda(m+1)} \int_{T-2 T_{1}}^{T} \int_{\mathcal{O}} \eta\left|\partial_{t} \theta\right| \xi|w|^{2} \leq C s^{2} \lambda^{3} e^{6 \lambda(m+1)} \int_{T-2 T_{1}}^{T} \int_{\mathcal{O}} \eta \theta^{2} \xi|w|^{2} \leq C s^{3} \lambda^{4} \iint_{\widehat{\omega}_{T}} \xi^{3}|w|^{2} .
$$

Hence, by combining the above estimates with (A.48), for some $s_{4} \geq s_{3}$ and $\lambda_{7} \geq \lambda_{6}$, there exists a constant $C$ such that for all $s \geq s_{4}$ and $\lambda \geq \lambda_{7}$,

$$
\begin{aligned}
& \int_{\mathcal{O}}|\nabla w(0)|^{2}+s^{2} \lambda^{3} e^{\lambda(12 m+2)} \int_{\mathcal{O}}|w(0)|^{2}+s \lambda^{2} \iint_{\mathcal{O}_{T}} \xi|\nabla w|^{2}+s^{3} \lambda^{4} \iint_{\mathcal{O}_{T}} \xi^{3}|w|^{2}+s^{2} \lambda^{2} \int_{0}^{T_{0}} \int_{\mathcal{O}}\left|\partial_{t} \theta\right| \xi \varphi|w|^{2} \\
& \quad \leq C \iint_{\mathcal{O}_{T}}|f|^{2} e^{-2 s \varphi}+C s^{3} \lambda^{4} \iint_{\widehat{\omega}_{T}} \xi^{3}|w|^{2} .
\end{aligned}
$$

Back to the function $z$. We now go back to the function $z=w e^{s \varphi}$. For that, let us first remark that there exists a constant $C=C\left(\|\nabla \psi\|_{\infty}\right)$ such that for all $(t, x) \in(0, T) \times \mathcal{O}$, 


$$
\begin{aligned}
& |z|^{2} e^{-2 s \varphi}=|w|^{2}, \\
& |\nabla z|^{2} e^{-2 s \varphi} \leq 2|\nabla w|^{2}+2 s^{2}|\nabla \varphi|^{2}|w|^{2} \leq 2|\nabla w|^{2}+2 C s^{2} \lambda^{2} \xi^{2}|w|^{2} .
\end{aligned}
$$

We immediately deduce from (A.49) that for all $s \geq s_{4}$ and $\lambda \geq \lambda_{7}$, for some positive constant $C$,

$$
\begin{aligned}
& \int_{\mathcal{O}}|\nabla z(0)|^{2} e^{-2 s \varphi(0)}+s^{2} \lambda^{3} e^{\lambda(12 m+2)} \int_{\mathcal{O}}|z(0)|^{2} e^{-2 s \varphi(0)}+s \lambda^{2} \iint_{\mathcal{O}_{T}} \xi|\nabla z|^{2} e^{-2 s \varphi} \\
& \quad+s^{3} \lambda^{4} \iint_{\mathcal{O}_{T}} \xi^{3}|z|^{2} e^{-2 s \varphi}+s^{2} \lambda^{2} \int_{0}^{T_{0}} \int_{\mathcal{O}}\left|\partial_{t} \theta\right| \xi \varphi|z|^{2} e^{-2 s \varphi} \\
& \leq C \iint_{\mathcal{O}_{T}}|f|^{2} e^{-2 s \varphi}+C s^{3} \lambda^{4} \iint_{\widetilde{\omega_{T}}} \xi^{3}|z|^{2} e^{-2 s \varphi} .
\end{aligned}
$$

We conclude the proof of Theorem 2.5 by setting $s_{0}=s_{4}$ and $\lambda_{0}=\lambda_{7}$.

\section{A.2. Proof of Theorem 2.6}

We divide the proof in several steps.

A duality approach. To solve the control problem (2.26)-(2.27), we first rewrite the control problem under a weak form. Multiplying $y$ solution of (2.26) by smooth functions $z$ on $[0, T] \times \overline{\mathcal{O}}$ such that $z=0$ on $[0, T] \times \partial \mathcal{O}$, we get:

$$
\int_{\mathcal{O}} \bar{\sigma}(T) y(T) z(T)+\iint_{\mathcal{O}_{T}} y\left(-\bar{\sigma} \partial_{t} z-\Delta z\right)=\iint_{\mathcal{O}_{T}} f z+\iint_{\widehat{\omega}_{T}} h z .
$$

In particular, since $\bar{\sigma}(T)>0$, the null-controllability requirement (2.27) is satisfied if and only if for all smooth functions $z$ on $[0, T] \times \overline{\mathcal{O}}$ such that $z=0$ on $[0, T] \times \partial \mathcal{O}$,

$$
\iint_{\mathcal{O}_{T}} y\left(-\bar{\sigma} \partial_{t} z-\Delta z\right)=\iint_{\mathcal{O}_{T}} f z+\iint_{\widehat{\omega}_{T}} h z
$$

The trick now is to introduce a functional $J$ whose Euler Lagrange equation coincide with (A.52): For smooth functions $z$ on $[0, T] \times \overline{\mathcal{O}}$ such that $z=0$ on $[0, T] \times \partial \mathcal{O}$, we define

$$
J(z)=\frac{1}{2} \iint_{\mathcal{O}_{T}}\left|\left(-\bar{\sigma} \partial_{t}-\Delta\right) z\right|^{2} e^{-2 s \varphi}+\frac{s^{3} \lambda^{4}}{2} \iint_{\widehat{\omega}_{T}} \xi^{3}|z|^{2} e^{-2 s \varphi}-\iint_{\mathcal{O}_{T}} f z .
$$

But the set of smooth functions $z$ on $[0, T] \times \overline{\mathcal{O}}$ such that $z=0$ on $[0, T] \times \partial \mathcal{O}$ is not a Banach space. We thus introduce

$$
X_{o b s}=\overline{\left\{z \in C^{\infty}([0, T] \times \overline{\mathcal{O}}) \text { such that } z=0 \text { on }[0, T] \times \partial \mathcal{O}\right\}}\|\cdot\|_{o b s}
$$

where $\|\cdot\|_{o b s}$ is the Hilbert norm defined by

$$
\|z\|_{o b s}^{2}=\iint_{\mathcal{O}_{T}}\left|\left(-\bar{\sigma} \partial_{t}-\Delta\right) z\right|^{2} e^{-2 s \varphi}+s^{3} \lambda^{4} \iint_{\widehat{\omega}_{T}} \xi^{3}|z|^{2} e^{-2 s \varphi} .
$$

The set $X_{o b s}$ is then endowed with the Hilbert structure given by $\|\cdot\|_{o b s}$. Note that here we use the fact that $\|\cdot\|_{o b s}$ is a norm, which is a consequence of the Carleman estimate (2.25). Also note that $X_{o b s}$ and $\|\cdot\|_{o b s}$ strongly depends on $s$ and $\lambda$ and we shall follow these dependences carefully in the sequel.

The functional $J$ can be extended as a continuous functional on $X_{o b s}$ provided (2.28) holds. Indeed, due to (2.25), we easily have, for some constant $C>0$ independent of $s$ and $\lambda$, 


$$
\left|\iint_{\mathcal{O}_{T}} f z\right| \leq C\|z\|_{o b s}\left(\frac{1}{s^{3} \lambda^{4}} \iint_{\mathcal{O}_{T}} \xi^{-3}|f|^{2} e^{2 s \varphi}\right)^{1 / 2} .
$$

It follows that, if condition (2.28) is satisfied, the functional $J$ can be uniquely extended as a continuous functional (still denoted the same) on $X_{o b s}$. Besides, (A.56) also implies the coercivity of $J$ on $X_{o b s}$. Since it is also strictly convex on $X_{o b s}$ since $\|\cdot\|_{o b s}$ is a Hilbert norm, $J$ admits a unique minimizer $Z$ on $X_{o b s}$.

Setting

$$
Y=\left(-\bar{\sigma} \partial_{t}-\Delta\right) Z e^{-2 s \varphi} \text { and } H=-s^{3} \lambda^{4} \xi^{3} Z e^{-2 s \varphi} 1_{\widehat{\omega}_{T}},
$$

writing the Euler Lagrange equation of $J$ at $Z$, for all smooth functions $z$ on $[0, T] \times \overline{\mathcal{O}}$ such that $z=0$ on $[0, T] \times \partial \mathcal{O}$,

$$
0=\iint_{\mathcal{O}_{T}} Y\left(-\bar{\sigma} \partial_{t} z-\Delta z\right)-\iint_{\widehat{\omega}_{T}} H z-\iint_{\mathcal{O}_{T}} f z,
$$

which coincides with (A.52).

In particular, (A.58) holds for all smooth functions $z$ on $[0, T] \times \overline{\mathcal{O}}$ such that $z=0$ on $[0, T] \times \partial \mathcal{O}$ with $z(T) \equiv 0$, which implies that $Y$ solves Eq. (2.26) with $h=H$ in the sense of transposition. By uniqueness of solutions in the sense of transposition, this is the solution of (2.26) in the classical sense. In particular, since $H \in L^{2}\left(\mathcal{O}_{T}\right), Y$ is $C\left([0, T] ; L^{2}(\mathcal{O})\right)$. Then, using again (A.58), we remark that it coincides with (A.52), hence $Y$ solves the control requirement (2.27).

Besides, using (A.56) and the fact that $J(Z) \leq J(0)=0$,

$$
s^{3} \lambda^{4} \iint_{\mathcal{O}_{T}}|Y|^{2} e^{2 s \varphi}+\iint_{\widehat{\omega}_{T}} \xi^{-3}|H|^{2} e^{2 s \varphi} \leq C \iint_{\mathcal{O}_{T}} \xi^{-3}|f|^{2} e^{2 s \varphi} .
$$

Estimates on $\nabla Y$. In the previous step, we found $(Y, H)$ satisfying the equations

$$
\begin{cases}\partial_{t}(\bar{\sigma} Y)-\Delta Y=f+H 1_{\widehat{\omega}_{T}}, & \text { in } \mathcal{O}_{T} \\ Y=0, & \text { in } \Gamma_{T} \\ Y(0, \cdot)=0, & \text { in } \mathcal{O}, \\ Y(T, \cdot)=0, & \text { in } \mathcal{O}\end{cases}
$$

and the estimates (A.59).

Our goal now is to obtain an estimate on $\nabla Y$. In order to do this, for $\varepsilon>0$, we introduce

$$
\varphi_{\varepsilon}(t, x): \stackrel{\text { def }}{=} \theta_{\varepsilon}(t)\left(\lambda e^{6 \lambda(m+1)}-e^{\psi(t, x)}\right), \quad \xi_{\varepsilon}(t): \stackrel{\text { def }}{=} \theta_{\varepsilon}(t) e^{\psi(t, x)}
$$

and $\theta_{\varepsilon}$ is given by:

$$
\theta_{\varepsilon}: \stackrel{\text { def }}{=} \theta_{\varepsilon}(t) \quad \text { such that }\left\{\begin{array}{l}
\forall t \in\left[0, T_{0}\right], \quad \theta_{\varepsilon}(t)=1+\left(1-\frac{t}{T_{0}}\right)^{\mu}, \\
\forall t \in\left[T_{0}, T-2 T_{1}+\varepsilon\right], \quad \theta_{\varepsilon}(t)=1, \\
\forall t \in\left[T-2 T_{1}+\varepsilon, T\right), \quad \theta_{\varepsilon}(t)=\theta(t-\varepsilon), \\
\mu \text { as in }(2.12) .
\end{array}\right.
$$

We then multiply Eq. (A.60) by $\xi_{\varepsilon}^{-2} Y e^{2 s \varphi_{\varepsilon}}$ :

$$
\begin{aligned}
- & \frac{1}{2} \iint_{\mathcal{O}_{T}}|Y|^{2} \partial_{t}\left(\bar{\sigma} \xi_{\varepsilon}^{-2} e^{2 s \varphi_{\varepsilon}}\right)+\iint_{\mathcal{O}_{T}}|Y|^{2} \partial_{t} \bar{\sigma} \xi_{\varepsilon}^{-2} e^{2 s \varphi_{\varepsilon}}+\iint_{\mathcal{O}_{T}} \xi_{\varepsilon}^{-2}|\nabla Y|^{2} e^{2 s \varphi_{\varepsilon}}-\frac{1}{2} \iint_{\mathcal{O}_{T}}|Y|^{2} \Delta\left(\xi_{\varepsilon}^{-2} e^{2 s \varphi_{\varepsilon}}\right) \\
& =\iint_{\mathcal{O}_{T}} f \xi_{\varepsilon}^{-2} Y e^{2 s \varphi_{\varepsilon}}+\iint_{\widehat{\omega}_{T}} H \xi_{\varepsilon}^{-2} Y e^{2 s \varphi_{\varepsilon}} .
\end{aligned}
$$

Following, multiplying by $s \lambda^{2}$, 


$$
\begin{aligned}
& s \lambda^{2} \iint_{\mathcal{O}_{T}} \xi_{\varepsilon}^{-2}|\nabla Y|^{2} e^{2 s \varphi_{\varepsilon}}-\frac{s \lambda^{2}}{2} \int_{0}^{T_{0}} \int_{\mathcal{O}} \bar{\sigma}|Y|^{2} \partial_{t}\left(\xi_{\varepsilon}^{-2} e^{2 s \varphi_{\varepsilon}}\right) \\
& =\frac{s \lambda^{2}}{2} \int_{T_{0}}^{T} \int_{\mathcal{O}} \bar{\sigma}|Y|^{2} \partial_{t}\left(\xi_{\varepsilon}^{-2} e^{2 s \varphi_{\varepsilon}}\right)+\frac{s \lambda^{2}}{2} \iint_{\mathcal{O}_{T}}|Y|^{2} \Delta\left(\xi_{\varepsilon}^{-2} e^{2 s \varphi_{\varepsilon}}\right) \\
& \quad+s \lambda^{2} \iint_{\mathcal{O}_{T}} f \xi_{\varepsilon}^{-2} e^{2 s \varphi_{\varepsilon}} Y+s \lambda^{2} \iint_{\widehat{\omega}_{T}} H \xi_{\varepsilon}^{-2} Y e^{2 s \varphi_{\varepsilon}}-\frac{s \lambda^{2}}{2} \iint_{\mathcal{O}_{T}}|Y|^{2} \partial_{t} \bar{\sigma} \xi_{\varepsilon}^{-2} e^{2 s \varphi_{\varepsilon}} .
\end{aligned}
$$

We then compute explicitly:

$$
-e^{-2 s \varphi_{\varepsilon}} \partial_{t}\left(\xi_{\varepsilon}^{-2} e^{2 s \varphi_{\varepsilon}}\right)=2 s \lambda \xi_{\varepsilon}^{-1} \partial_{t} \psi-2 s \xi_{\varepsilon}^{-2} \frac{\partial_{t} \theta_{\varepsilon}}{\theta_{\varepsilon}} \varphi_{\varepsilon}+2 \frac{\partial_{t} \theta_{\varepsilon}}{\theta_{\varepsilon}} \xi_{\varepsilon}^{-2}+2 \lambda \partial_{t} \psi \xi_{\varepsilon}^{-2}
$$

On $\left(0, T_{0}\right)$, we remove the dependence in $\varepsilon>0$ as $\theta_{\varepsilon}=\theta$ on $\left(0, T_{0}\right)$. Using (2.13), $\partial_{t} \theta \leq 0$ and $\theta \in[1,2]$ in $\left[0, T_{0}\right]$ we have, for all $s \geq s_{0}$ and $t \in\left(0, T_{0}\right)$,

$$
-2 s \xi^{-2} \frac{\partial_{t} \theta}{\theta} \varphi+2 \frac{\partial_{t} \theta}{\theta} \xi^{-2} \geq c s\left|\partial_{t} \theta\right| \xi^{-2} \varphi
$$

whereas

$$
\left|2 s \lambda \xi^{-1} \partial_{t} \psi+2 \lambda \partial_{t} \psi \xi^{-2}\right| \leq C s \lambda \xi^{-1}
$$

Hence

$$
-\frac{s \lambda^{2}}{2} \int_{0}^{T_{0}} \int_{\mathcal{O}} \bar{\sigma}|Y|^{2} \partial_{t}\left(\xi_{\varepsilon}^{-2} e^{2 s \varphi_{\varepsilon}}\right) \geq c s^{2} \lambda^{2} \int_{0}^{T_{0}} \int_{\mathcal{O}} \bar{\sigma}\left|\partial_{t} \theta\right| \xi^{-2} \varphi|Y|^{2} e^{2 s \varphi}-C s^{2} \lambda^{3} \int_{0}^{T_{0}} \int_{\mathcal{O}}|Y|^{2} e^{2 s \varphi} .
$$

On $\left(T_{0}, T\right)$, from the identity (A.62), using $\left|\partial_{t} \theta_{\varepsilon}\right| \leq C \theta_{\varepsilon}^{2}$, we derive

$$
\left|2 s \lambda \xi_{\varepsilon}^{-2} \partial_{t} \psi-2 s \xi_{\varepsilon}^{-2} \frac{\partial_{t} \theta_{\varepsilon}}{\theta_{\varepsilon}} \varphi+2 \frac{\partial_{t} \theta_{\varepsilon}}{\theta_{\varepsilon}} \xi_{\varepsilon}^{-2}+2 \lambda \partial_{t} \psi \xi_{\varepsilon}^{-2}\right| \leq C s \lambda
$$

We thus obtain

$$
\left.\left.\left|\frac{s \lambda^{2}}{2} \int_{T_{0}}^{T} \int_{\mathcal{O}} \bar{\sigma}\right| Y\right|^{2} \partial_{t}\left(\xi_{\varepsilon}^{-2} e^{2 s \varphi_{\varepsilon}}\right)\left|\leq C s^{2} \lambda^{3} \int_{T_{0}}^{T} \int_{\mathcal{O}}\right| Y\right|^{2} e^{2 s \varphi_{\varepsilon}} .
$$

Straightforward computations yield $\left|\Delta\left(\xi_{\varepsilon}^{-2} e^{2 s \varphi_{\varepsilon}}\right)\right| \leq C s^{2} \lambda^{2} e^{2 s \varphi_{\varepsilon}}$, from which we get

$$
\left.\left.\left|\frac{s \lambda^{2}}{2} \iint_{\mathcal{O}_{T}}\right| Y\right|^{2} \Delta\left(\xi_{\varepsilon}^{-2} e^{2 s \varphi_{\varepsilon}}\right)\left|\leq C s^{3} \lambda^{4} \iint_{\mathcal{O}_{T}}\right| Y\right|^{2} e^{2 s \varphi_{\varepsilon}} .
$$

Using Cauchy-Schwarz estimates,

$$
\left|s \lambda^{2} \iint_{\mathcal{O}_{T}}\left(\left(f+H 1_{\widehat{\omega}_{T}}\right) \xi_{\varepsilon}^{-2} e^{2 s \varphi_{\varepsilon}}\right) Y\right| \leq s^{3} \lambda^{4} \iint_{\mathcal{O}_{T}}|Y|^{2} e^{2 s \varphi_{\varepsilon}}+\frac{C}{s} \iint_{\mathcal{O}_{T}} \xi_{\varepsilon}^{-4}|f|^{2} e^{2 s \varphi_{\varepsilon}}+\frac{C}{s} \int_{\widehat{\omega}_{T}} \xi_{\varepsilon}^{-4}|H|^{2} e^{2 s \varphi_{\varepsilon}} .
$$

Since we obviously have

$$
\left.\left.\left|s \lambda^{2} \iint_{\mathcal{O}_{T}}\right| Y\right|^{2} \partial_{t} \bar{\sigma} \xi_{\varepsilon}^{-2} e^{2 s \varphi_{\varepsilon}}\left|\leq C s^{3} \lambda^{4} \iint_{\mathcal{O}_{T}}\right| Y\right|^{2} e^{2 s \varphi_{\varepsilon}},
$$


combining estimates (A.63)-(A.67) and plugging (A.61), we obtain

$$
\begin{aligned}
& s \lambda^{2} \iint_{\mathcal{O}_{T}} \xi_{\varepsilon}^{-2}|\nabla Y|^{2} e^{2 s \varphi_{\varepsilon}}+s^{2} \lambda^{2} \int_{0}^{T_{0}} \int_{\mathcal{O}} \bar{\sigma}\left|\partial_{t} \theta\right| \xi^{-2}|Y|^{2} e^{2 s \varphi} \\
& \leq C s^{3} \lambda^{4} \iint_{\mathcal{O}_{T}}|Y|^{2} e^{2 s \varphi_{\varepsilon}}+C \iint_{\widehat{\omega}_{T}} \xi_{\varepsilon}^{-3}|H|^{2} e^{2 s \varphi_{\varepsilon}}+C \iint_{\mathcal{O}_{T}} \xi_{\varepsilon}^{-3}|f|^{2} e^{2 s \varphi_{\varepsilon}} .
\end{aligned}
$$

Since the constant $C$ is independent of $\varepsilon>0$, we can pass to the limit $\varepsilon \rightarrow 0$, and using (A.59) and the fact that $\bar{\sigma}$ is bounded from below away from 0 , we get:

$$
s \lambda^{2} \iint_{\mathcal{O}_{T}} \xi^{-2}|\nabla Y|^{2} e^{2 s \varphi}+s^{2} \lambda^{2} \int_{0}^{T_{0}} \int_{\mathcal{O}}\left|\partial_{t} \theta\right| \xi^{-2} \varphi|Y|^{2} e^{2 s \varphi} \leq C \iint_{\mathcal{O}_{T}} \xi^{-3}|f|^{2} e^{2 s \varphi} .
$$

Estimates on $\Delta Y, \partial_{t} Y$. Multiplying Eq. (A.60) by $-\xi_{\varepsilon}^{-4} \Delta Y e^{2 s \varphi_{\varepsilon}} / s$, we obtain

$$
\begin{aligned}
& -\frac{1}{2 s} \iint_{\mathcal{O}_{T}} \partial_{t}\left(\bar{\sigma} \xi_{\varepsilon}^{-4} e^{2 s \varphi_{\varepsilon}}\right)|\nabla Y|^{2}+\frac{1}{s} \iint_{\mathcal{O}_{T}} \partial_{t} Y \nabla Y \cdot \nabla\left(\bar{\sigma} \xi_{\varepsilon}^{-4} e^{2 s \varphi_{\varepsilon}}\right) \\
& +\frac{1}{s} \iint_{\mathcal{O}_{T}} \xi_{\varepsilon}^{-4}|\Delta Y|^{2} e^{2 s \varphi_{\varepsilon}}=-\frac{1}{s} \iint_{\mathcal{O}_{T}}\left(f+V 1_{\widehat{\omega}_{T}}-\partial_{t} \bar{\sigma} Y\right) \xi_{\varepsilon}^{-4} \Delta Y e^{2 s \varphi_{\varepsilon}} .
\end{aligned}
$$

As in (A.62), we compute explicitly $-\partial_{t}\left(\xi_{\varepsilon}^{-4} e^{2 s \varphi_{\varepsilon}}\right)$. Arguing as in (A.63), we get

$$
-\frac{1}{2 s} \int_{0}^{T_{0}} \int_{\mathcal{O}} \bar{\sigma} \partial_{t}\left(\xi_{\varepsilon}^{-4} e^{2 s \varphi_{\varepsilon}}\right)|\nabla Y|^{2} \geq c \int_{0}^{T_{0}} \int_{\mathcal{O}} \bar{\sigma}\left|\partial_{t} \theta\right| \xi^{-4} \varphi|\nabla Y|^{2} e^{2 s \varphi}-C \lambda \int_{0}^{T_{0}} \int_{\mathcal{O}} \xi^{-2}|\nabla Y|^{2} e^{2 s \varphi} .
$$

Besides, arguing as in (A.64), we get

$$
\left.\left.\left|-\frac{1}{2 s} \int_{T_{0}}^{T} \int_{\mathcal{O}} \bar{\sigma} \partial_{t}\left(\xi_{\varepsilon}^{-4} e^{2 s \varphi_{\varepsilon}}\right)\right| \nabla Y\right|^{2}\left|\leq C s \lambda^{2} \iint_{\mathcal{O}_{T}} \xi_{\varepsilon}^{-2}\right| \nabla Y\right|^{2} e^{2 s \varphi_{\varepsilon}} .
$$

One can also easily check that

$$
\left.\left.\left|-\frac{1}{2 s} \iint_{\mathcal{O}_{T}} \partial_{t} \bar{\sigma}\left(\xi_{\varepsilon}^{-4} e^{2 s \varphi_{\varepsilon}}\right)\right| \nabla Y\right|^{2}\left|\leq C s \lambda^{2} \iint_{\mathcal{O}_{T}} \xi_{\varepsilon}^{-2}\right| \nabla Y\right|^{2} e^{2 s \varphi_{\varepsilon}} .
$$

We then estimate the cross-term of (A.69):

$$
\left|\frac{1}{s} \iint_{\mathcal{O}_{T}} \partial_{t} Y \nabla Y \cdot \nabla\left(\bar{\sigma} \xi_{\varepsilon}^{-4} e^{2 s \varphi_{\varepsilon}}\right)\right| \leq \frac{\bar{\sigma}_{\min }^{2}}{8 s} \iint_{\mathcal{O}_{T}} \xi_{\varepsilon}^{-4}\left|\partial_{t} Y\right|^{2} e^{2 s \varphi_{\varepsilon}}+C s \lambda^{2} \iint_{\mathcal{O}_{T}} \xi_{\varepsilon}^{-2}|\nabla Y|^{2} e^{2 s \varphi_{\varepsilon}},
$$

where $\bar{\sigma}_{\min }: \stackrel{\text { def }}{=} \min \frac{\mathcal{O}_{T}}{\sigma}$. From Eq. (A.60),

$$
\partial_{t} Y=\frac{1}{\bar{\sigma}}\left(\Delta Y+f+H 1_{\omega_{T}}-\partial_{t} \bar{\sigma} Y\right),
$$

and thus we deduce

$$
\begin{aligned}
\frac{\bar{\sigma}_{\min }^{2}}{8 s} \iint_{\mathcal{O}_{T}} \xi_{\varepsilon}^{-4}\left|\partial_{t} Y\right|^{2} e^{2 s \varphi_{\varepsilon}} \leq & \frac{1}{4 s} \iint_{\mathcal{O}_{T}} \xi_{\varepsilon}^{-4}|\Delta Y|^{2} e^{2 s \varphi_{\varepsilon}}+\frac{C}{s} \iint_{\mathcal{O}_{T}}|Y|^{2} e^{2 s \varphi_{\varepsilon}} \\
& +\frac{C}{s} \iint_{\mathcal{O}_{T}} \xi_{\varepsilon}^{-3}|f|^{2} e^{2 s \varphi_{\varepsilon}}+\frac{C}{s} \iint_{\widehat{\omega}_{T}} \xi_{\varepsilon}^{-3}|H|^{2} e^{2 s \varphi_{\varepsilon}} .
\end{aligned}
$$


And of course,

$$
\begin{aligned}
\left|-\frac{1}{s} \iint_{\mathcal{O}_{T}}\left(f+H 1_{\omega_{T}}-\partial_{t} \bar{\sigma} Y\right) \xi_{\varepsilon}^{-4} \Delta Y e^{2 s \varphi_{\varepsilon}}\right| \leq & \frac{1}{4 s} \iint_{\mathcal{O}_{T}} \xi_{\varepsilon}^{-4}|\Delta Y|^{2} e^{2 s \varphi_{\varepsilon}}+\frac{C}{s} \iint_{\mathcal{O}_{T}} \xi_{\varepsilon}^{-3}|f|^{2} e^{2 s \varphi_{\varepsilon}} \\
& +\frac{C}{s} \iint_{\bar{\omega}_{T}} \xi_{\varepsilon}^{-3}|H|^{2} e^{2 s \varphi_{\varepsilon}}+\frac{C}{s} \iint_{\mathcal{O}_{T}}|Y|^{2} e^{2 s \varphi_{\varepsilon}} .
\end{aligned}
$$

Combining all the above estimates, we get

$$
\begin{aligned}
\frac{1}{2 s} \iint_{\mathcal{O}_{T}} \xi_{\varepsilon}^{-4}|\Delta Y|^{2} e^{2 s \varphi_{\varepsilon}} \leq & C s \lambda^{2} \iint_{\mathcal{O}_{T}} \xi_{\varepsilon}^{-2}|\nabla Y|^{2} e^{2 s \varphi_{\varepsilon}}+C s^{3} \lambda^{4} \iint_{\mathcal{O}_{T}}|Y|^{2} e^{2 s \varphi_{\varepsilon}} \\
& +C \iint_{\mathcal{O}_{T}} \xi_{\varepsilon}^{-3}|f|^{2} e^{2 s \varphi_{\varepsilon}}+C \iint_{\bar{\omega}_{T}} \xi_{\varepsilon}^{-3}|H|^{2} e^{2 s \varphi_{\varepsilon}} .
\end{aligned}
$$

Since the constant $C$ does not depend on $\varepsilon>0$, we can pass to the limit $\varepsilon \rightarrow 0$ :

$$
\begin{aligned}
\frac{1}{2 s} \iint_{\mathcal{O}_{T}} \xi^{-4}|\Delta Y|^{2} e^{2 s \varphi} \leq & C s \lambda^{2} \iint_{\mathcal{O}_{T}} \xi^{-2}|\nabla Y|^{2} e^{2 s \varphi}+C s^{3} \lambda^{4} \iint_{\mathcal{O}_{T}}|Y|^{2} e^{2 s \varphi} \\
& +C \iint_{\mathcal{O}_{T}} \xi^{-3}|f|^{2} e^{2 s \varphi}+C \iint_{\widehat{\omega}_{T}} \xi^{-3}|H|^{2} e^{2 s \varphi}
\end{aligned}
$$

Using now estimates (A.59), (A.68) and (A.75), we get

$$
\frac{1}{s} \iint_{\mathcal{O}_{T}} \xi^{-4}\left(\left|\partial_{t} Y\right|^{2}+|\Delta Y|^{2}\right) e^{2 s \varphi} \leq C \iint_{\mathcal{O}_{T}} \xi^{-3}|f|^{2} e^{2 s \varphi} .
$$

Estimates on $\partial_{\mathbf{n}} Y$ in $L^{2}\left(\Gamma_{T}\right)$. Let $\eta: \overline{\mathcal{O}} \mapsto \mathbb{R}^{d}$ such that $\eta \in C^{2}\left(\overline{\mathcal{O}} ; \mathbb{R}^{d}\right)$ and $\eta=\vec{n}$ on $\partial \mathcal{O}$. Since $Y$ vanishes on $\Gamma_{T}$, we have the following identity: for all $\varepsilon>0$,

$$
\begin{aligned}
\frac{1}{2} \int_{\Gamma_{T}} \xi_{\varepsilon}^{-3}\left|\partial_{\mathbf{n}} Y\right|^{2} e^{2 s \varphi_{\varepsilon}}= & \iint_{\mathcal{O}_{T}} \xi_{\varepsilon}^{-3} \Delta Y \eta \cdot \nabla Y e^{2 s \varphi_{\varepsilon}} \\
& +\iint_{\mathcal{O}_{T}} D\left(\xi_{\varepsilon}^{-3} \eta e^{2 s \varphi_{\varepsilon}}\right)(\nabla Y, \nabla Y)-\frac{1}{2} \iint_{\mathcal{O}_{T}} \operatorname{div}\left(\eta \xi_{\varepsilon}^{-3} e^{2 s \varphi_{\varepsilon}}\right)|\nabla Y|^{2} .
\end{aligned}
$$

Hence

$$
\lambda \int_{\Gamma_{T}} \xi_{\varepsilon}^{-3}\left|\partial_{\mathbf{n}} Y\right|^{2} e^{2 s \varphi_{\varepsilon}} \leq \frac{1}{s} \iint_{\mathcal{O}_{T}} \xi_{\varepsilon}^{-4}|\Delta Y|^{2} e^{2 s \varphi_{\varepsilon}}+C s \lambda^{2} \iint_{\mathcal{O}_{T}} \xi_{\varepsilon}^{-2}|\nabla Y|^{2} e^{2 s \varphi_{\varepsilon}} .
$$

Passing to the limit in $\varepsilon \rightarrow 0$ and using (A.68) and (A.77) we thus obtain

$$
\lambda \int_{\Gamma_{T}} \xi^{-3}\left|\partial_{\mathbf{n}} Y\right|^{2} e^{2 s \varphi} \leq C \iint_{\mathcal{O}_{T}} \xi^{-3}|f|^{2} e^{2 s \varphi} .
$$

Conclusion. Estimates (A.59), (A.68), (A.77) and (A.78) yield (2.29).

\section{Appendix B. Regularity of the weight function}

Proof of Lemma 4.5. The first remark is that the flow $\bar{X}_{e}$ is $\mathbf{C}^{2}\left([0, T] \times[0, T] \times \mathbb{R}^{2}\right)$ since $\overline{\mathbf{y}}_{e} \in \mathbf{C}^{2}\left([0, T] \times \mathbb{R}^{2}\right)$. 
In order to study the regularity of $\widehat{\psi}$, we will introduce the function $t_{\text {out }}=t_{\text {out }}(t, x)$ defined for $(t, x) \in(0, T) \times \mathcal{O}$ as the supremum of the time $\tau \in(t, T]$ for which $\forall t^{\prime} \in(t, \tau), \bar{X}_{e}\left(t^{\prime}, t, x\right) \in \mathcal{O}$. It is not difficult to check that this time $t_{\text {out }}$ can also be characterized as the solution of

$$
\begin{cases}\partial_{t} t_{\text {out }}+\overline{\mathbf{y}}_{e} \cdot \nabla t_{\text {out }}=0 & \text { in } \mathcal{O}_{T}, \\ t_{\text {out }}(t)=t & \text { on } \Gamma_{T}, \\ t_{\text {out }}(T)=T & \text { in } \mathcal{O} .\end{cases}
$$

For convenience, we also set

$$
x_{\text {out }}(t, x)=\bar{X}_{e}\left(t_{\text {out }}(t, x), t, x\right) .
$$

We first prove that $t_{\text {out }}$ is continuous in $\mathcal{O}_{T}$. In order to do that, let us remark that $\bar{X}_{e}$ is $\mathbf{C}^{2}\left([0, T] \times[0, T] \times \mathbb{R}^{2}\right)$ and for all $(t, \tau) \in[0, T]^{2}, \bar{X}_{e}(t, \tau, \cdot)$ is a $C^{2}$ diffeomorphism of $\mathbb{R}^{2}$. In particular, $\mathcal{O}_{T}$ can be decomposed into

$$
\begin{aligned}
& \mathcal{O}_{T}=\mathcal{O}_{T, 1} \cup \mathcal{O}_{T, 2} \cup \Sigma_{T}, \\
& \text { with }\left\{\begin{array}{l}
\mathcal{O}_{T, 1}=\left\{(t, x) \in(0, T) \times \mathcal{O}, x \in \bar{X}_{e}(t, T, \mathcal{O})\right\}, \\
\mathcal{O}_{T, 2}=\left\{(t, x) \in(0, T) \times \mathcal{O}, x \in \bar{X}_{e}\left(t, T, \mathbb{R}^{2} \backslash \overline{\mathcal{O}}\right)\right\}, \\
\Sigma_{T}=\left\{(t, x) \in(0, T) \times \mathcal{O}, x \in \bar{X}_{e}(t, T, \partial \mathcal{O})\right\} .
\end{array}\right.
\end{aligned}
$$

In (B.3), $\mathcal{O}_{T, 1}$ and $\mathcal{O}_{T, 2}$ are open sets whereas $\Sigma_{T}=\overline{\mathcal{O}_{T, 1}} \cap \overline{\mathcal{O}_{T, 2}}$ is closed and of dimension 2. For $(t, x) \in \mathcal{O}_{T, 1} \cup \Sigma_{T}$, $t_{\text {out }}(t, x)=T$ and $t_{\text {out }}$ is thus continuous on $\overline{\mathcal{O}_{T, 1}}$. The continuity on $\overline{\mathcal{O}_{T, 2}}$ is more involved. If $(t, x) \in \overline{\mathcal{O}_{T, 2}}$, then $x_{\text {out }}(t, x)$ belongs to $\partial \mathcal{O}$. Due to the condition (4.5), for any $\varepsilon>0$, there exists a neighborhood $\mathscr{V}_{\varepsilon}$ of $\left(t_{\text {out }}(t, x), x_{\text {out }}(t, x)\right)$ in $[0, T] \times \overline{\mathcal{O}}$ such that $\left|t_{\text {out }}\left(t^{\prime}, x^{\prime}\right)-t_{\text {out }}(t, x)\right|<\varepsilon$ for all $\left(t^{\prime}, x^{\prime}\right) \in \mathscr{V}_{\varepsilon}$. In particular, for some $t_{\varepsilon} \in(0, T)$ close to $t_{\text {out }}(t, x), \mathscr{V}_{\varepsilon}$ is a neighborhood of $\left(t_{\varepsilon}, \bar{X}_{e}\left(t_{\varepsilon}, t_{\text {out }}(t, x), x_{\text {out }}(t, x)\right)\right)=\left(t_{\varepsilon}, \bar{X}_{e}\left(t_{\varepsilon}, t, x\right)\right)$. Following, $\left\{\bar{X}_{e}\left(t-t_{\varepsilon}+t^{\prime}, t^{\prime}, x^{\prime}\right),\left(t^{\prime}, x^{\prime}\right) \in \mathscr{V}_{\varepsilon}\right\}$ is a neighborhood of $\left(t, \bar{X}_{e}\left(t, t_{\varepsilon}, \bar{X}_{e}\left(t_{\varepsilon}, t, x\right)\right)\right)=(t, x)$ on which $t_{\text {out }}$ is at distance at most $\varepsilon$ of $t_{\text {out }}(t, x)$.

Thus, $t_{\text {out }}$ is continuous in $\overline{\mathcal{O}_{T}}$. As $\widehat{\psi}$ solution of (4.8) can be written as

$$
\widehat{\psi}(t, x)= \begin{cases}\widehat{\psi}_{T}\left(x_{\text {out }}(t, x)\right) & \text { if } t_{\text {out }}(t, x)=T, \\ t_{\text {out }}(t, x)-T & \text { if } t_{\text {out }}(t, x)<T,\end{cases}
$$

the continuity of $\widehat{\psi}$ in $\overline{\mathcal{O}_{T}}$ follows from the first compatibility condition in (4.7). Also note that $\widehat{\psi}$ is obviously $C^{2}$ in $\mathcal{O}_{T, 1}$.

We then focus on the $C^{1}$ regularity of $\widehat{\psi}$. In order to do this, we remark that $\nabla t_{\text {out }}$ solves

$$
\begin{cases}\partial_{t} \nabla t_{\text {out }}+\left(\overline{\mathbf{y}}_{e} \cdot \nabla\right) \nabla t_{\text {out }}+D \overline{\mathbf{y}}_{e} \nabla t_{\text {out }}=0 & \text { in } \mathcal{O}_{T}, \\ \nabla t_{\text {out }}(t, x)=-\frac{\mathbf{n}(x)}{\overline{\mathbf{y}}_{e}(t, x) \cdot \mathbf{n}(x)} & \text { on } \Gamma_{T}, \\ \nabla t_{\text {out }}(T)=0 & \text { in } \mathcal{O} .\end{cases}
$$

In particular, $\nabla t_{\text {out }}$ can be computed for any $(t, x) \in \mathcal{O}_{T, 2}$ by solving for $\tau$ between $t$ and $t_{\text {out }}(t, x)$ the ODE

$$
\begin{aligned}
& \frac{d}{d \tau}\left(\nabla t_{\text {out }}\left(\tau, \bar{X}_{e}(\tau, t, x)\right)\right)=-D \overline{\mathbf{y}}_{e}\left(\tau, \bar{X}_{e}(\tau, t, x)\right) \nabla t_{\text {out }}\left(\tau, \bar{X}_{e}(\tau, t, x)\right), \quad \tau \in\left(t, t_{\text {out }}(t, x)\right), \\
& \quad \text { with } \nabla t_{\text {out }}\left(t_{\text {out }}(t, x), x_{\text {out }}(t, x)\right)=-\frac{\mathbf{n}\left(x_{\text {out }}(t, x)\right)}{\overline{\mathbf{y}}_{e}\left(t_{\text {out }}(t, x), x_{\text {out }}(t, x)\right) \cdot \mathbf{n}\left(x_{\text {out }}(t, x)\right)} .
\end{aligned}
$$

One then easily obtains that $\nabla t_{\text {out }}$ is $C^{0}$ on $\mathcal{O}_{T, 2}$ and from Eq. (B.1) we deduce that $t_{\text {out }}$ is $C^{1}$ in $\mathcal{O}_{T, 2}$. From there, we derived immediately from (B.4) that $\widehat{\psi}$ is $C^{1}$ on $\mathcal{O}_{T, 2}$ and that it can be extended as a $C^{1}$ function on $\overline{\mathcal{O}_{T, 2}}$ as follows: $\nabla \widehat{\psi}$ can be computed for any $(t, x) \in \Sigma_{T}$ by solving for $\tau$ between $t$ and $T$ the ODE:

$$
\begin{aligned}
\frac{d}{d \tau}\left(\nabla \widehat{\psi}\left(\tau, \bar{X}_{e}(\tau, t, x)\right)\right) & =-D \overline{\mathbf{y}}_{e}\left(\tau, \bar{X}_{e}(\tau, t, x)\right) \nabla \widehat{\psi}\left(\tau, \bar{X}_{e}(\tau, t, x)\right), \quad \tau \in(t, T), \\
\text { with } \nabla \widehat{\psi}\left(T, \bar{X}_{e}(T, t, x)\right) & =-\frac{\mathbf{n}\left(\bar{X}_{e}(T, t, x)\right)}{\overline{\mathbf{y}}_{e}\left(T, \bar{X}_{e}(T, t, x)\right) \cdot \mathbf{n}\left(\bar{X}_{e}(T, t, x)\right)} .
\end{aligned}
$$


On the other hand, $\widehat{\psi}$ solves Eq. (4.11), and can be extended as a $C^{1}$ function on $\overline{\mathcal{O}_{T, 1}}$. For $(t, x) \in \Sigma_{T}$, this yields $\nabla \widehat{\psi}(t, x)$ as the solution of the $\operatorname{ODE}(\mathrm{B} .6)$ with $\nabla \widehat{\psi}\left(T, \bar{X}_{e}(T, t, x)\right)$ given. But, as $\widehat{\psi}(T)$ is constant on the boundary and satisfies the second compatibility condition in (4.5), we get again (B.7) for $(t, x) \in \Sigma_{T}$. Following, $\nabla \widehat{\psi}$ is continuous across $\Sigma_{T}$, hence on $\overline{\mathcal{O}_{T}}$. Using Eq. (4.8), $\widehat{\psi}$ belongs to $C^{1}\left(\overline{\mathcal{O}_{T}}\right)$.

The proof of the $C^{2}$ regularity follows the same path and is left to the reader.

\section{Appendix C. Proof of Theorem 1.2}

The proof of Theorem 1.2 follows the one of Theorem 1.1. The main difference is that we assume that we control on the whole boundary $(0, T) \times \partial \Omega$. In that case, the set $\Omega$ can be embedded into a large torus $\mathcal{O}=\mathbb{T}_{L}$, where $L$ is a large number corresponding to the size of the torus.

The control problem (2.4)-(2.5) for the velocity field $\mathbf{u}$ can then be set on a domain without boundary. Therefore, if we choose $\tilde{\psi}$ satisfying

$$
\left\{\begin{array}{l}
\forall(t, x) \in[0, T] \times \mathbb{T}_{L}, \quad \tilde{\psi}(t, x) \in[0,1], \\
\inf \left\{|\nabla \tilde{\psi}(t, x)|,(t, x) \in[0, T] \times \mathbb{T}_{L}, \text { s.t. } d(x, \Omega) \leq 3\right\} \geq \alpha>0,
\end{array}\right.
$$

the same strategy as the one developed for Theorem 2.1 applies, except that no boundary terms appear. In particular, estimate (2.37) holds without the presence of the boundary term. This means that, for all smooth $\mathbf{v}$ solution of (2.17), setting $w=\operatorname{curl} \mathbf{v}$, for all $s \geq s_{0}$ and $\lambda \geq \lambda_{0}$,

$$
\begin{aligned}
& s^{-1} \int_{0}^{T} \int_{\mathbb{T}_{L}} \xi^{2}|\nabla \mathbf{v}|^{2} e^{-2 s \varphi}+\int_{0} \int_{\mathbb{T}_{L}} \xi^{3}|w|^{2} e^{-2 s \varphi}+s \lambda^{2} \iint_{\mathcal{O}_{T}} \xi^{4}|\mathbf{v}|^{2} e^{-2 s \varphi} \\
& \quad \leq C\left(\iint_{\widehat{\omega_{T}}} \xi^{3}|w|^{2} e^{-2 s \varphi}+s \lambda^{2} \iint_{\widehat{\omega_{T}}} \xi^{4}|\mathbf{v}|^{2} e^{-2 s \varphi}+s^{-1} \lambda^{-2} \iint_{0}^{T} \xi_{\mathbb{T}_{L}} \xi^{2}|\mathbf{g}|^{2} e^{-2 s \varphi}\right),
\end{aligned}
$$

where $\widehat{\omega_{T}}=(0, T) \times\left\{x \in \mathbb{T}_{L}\right.$, s.t. $\left.d(x, \Omega) \geq 2\right\}$. As in the proof of Theorem 2.1, one can then remove the observation in $w$ and express it in terms of an observation in $\mathbf{v}$. This easily yields Theorem 2.1 with $\omega_{T}=(0, T) \times\{x \in$ $\mathbb{T}_{L}$, s.t. $\left.d(x, \Omega) \geq 1\right\}$ and the control result corresponding to Theorem 2.3.

Concerning the controllability of the density done in Section 3, one can basically do the same thing as in the 2-d case thanks to the Sobolev embedding $L^{2}\left(0, T ; \mathbf{H}^{2}\left(\mathbb{R}^{3}\right)\right) \subset L^{2}\left(0, T ; \mathbf{L}^{\infty}\left(\mathbb{R}^{3}\right)\right)$. However, one needs to be careful as the flow $\widehat{X}$ defined in (3.2) is not defined in the classical sense for a velocity field only in $L^{2}\left(0, T ; \mathbf{H}^{2}\left(\mathbb{R}^{3}\right)\right)$. One needs to consider the flow defined in the sense of [10], which solves Eq. (3.2) only almost everywhere. To avoid this technical difficulty, given $\widehat{\mathbf{u}} \in L^{2}\left(0, T ; \mathbf{H}^{2}(\Omega)\right)$ satisfying (3.18)-(3.19), we approximate it by a sequence $\widehat{\mathbf{u}}_{n}$ of $L^{2}\left(0, T ; \mathbf{H}^{3}(\Omega)\right)$ of divergence free vector fields satisfying (3.18)-(3.19) and such that $\xi^{-2} \widehat{\mathbf{u}}_{n} e^{s \varphi}$ is strongly convergent to $\xi^{-2} \widehat{\mathbf{u}} e^{s \varphi}$ in $L^{2}\left(0, T ; \mathbf{L}^{2}(\Omega)\right)$ and $\widehat{\mathbf{u}}_{n} e^{3 s \varphi^{*} / 4}$ strongly converges to $\widehat{\mathbf{u}} e^{3 s \varphi^{*} / 4}$ in $L^{2}\left(0, T ; \mathbf{H}^{2}(\Omega)\right)$. The construction of Section 3 then applies without any change for any $\widehat{\mathbf{u}}_{n}$, yielding a sequence of controlled densities $\rho_{n}$ solving the controlled problem (1.19) with the velocity field $\widehat{\mathbf{u}}_{n}$ satisfying the estimates of Theorem 3.6. Thanks to the convergence results in [2,3] and following the proof in Section 4.3, this construction shows the existence of a controlled density $\rho$ solving the controlled problem (1.19) with the estimates of Theorem 3.6. Besides, thanks to the convergence results in $[2,3]$, this controlled density $\rho$ coincides with the one in (3.9).

One can therefore conclude as in Section 4. The construction of $\tilde{\psi}$ satisfying (C.1) can be done as in the proof of Lemma 4.1: one can then simply take an extension $\overline{\mathbf{y}_{e}}$ of the velocity field vanishing for $x \in \mathbb{T}_{L}$ with $d(x, \Omega) \geq 3$, and choose an initial data $\tilde{\psi}_{T}$ in (4.8) having its critical points localized in the set $\left\{x \in \mathbb{T}_{L}\right.$, s.t. $\left.d(x, \Omega) \geq 3\right\}$. The rest of the proof of the fixed point argument is left unchanged.

\section{References}

[1] P. Albano, D. Tataru, Carleman estimates and boundary observability for a coupled parabolic-hyperbolic system, Electron. J. Differ. Equ. 22 (2000), 15 pp. (electronic). 
[2] F. Boyer, Trace theorems and spatial continuity properties for the solutions of the transport equation, Differ. Integral Equ. 18 (8) (2005) 891-934.

[3] F. Boyer, P. Fabrie, Outflow boundary conditions for the incompressible non-homogeneous Navier-Stokes equations, Discrete Contin. Dyn. Syst., Ser. B 7 (2) (2007) 219-250 (electronic).

[4] F. Boyer, P. Fabrie, Mathematical Tools for the Study of the Incompressible Navier-Stokes Equations and Related Models, Applied Mathematical Sciences, vol. 183, Springer, New York, 2013.

[5] F.W. Chaves-Silva, L. Rosier, E. Zuazua, Null controllability of a system of viscoelasticity with a moving control, http://arxiv.org/ abs/1303.3452.

[6] J.-M. Coron, On the controllability of the 2-D incompressible Navier-Stokes equations with the Navier slip boundary conditions, ESAIM Control Optim. Calc. Var. 1 (1995/96) 35-75 (electronic).

[7] J.-M. Coron, On the controllability of 2-D incompressible perfect fluids, J. Math. Pures Appl. (9) 75 (2) (1996) 155-188.

[8] J.-M. Coron, A.V. Fursikov, Global exact controllability of the 2D Navier-Stokes equations on a manifold without boundary, Russ. J. Math. Phys. 4 (4) (1996) 429-448.

[9] B. Desjardins, Linear transport equations with initial values in Sobolev spaces and application to the Navier-Stokes equations, Differ. Integral Equ. 10 (3) (1997) 577-586.

[10] R.J. DiPerna, P.-L. Lions, Ordinary differential equations, transport theory and Sobolev spaces, Invent. Math. 98 (3) (1989) $511-547$.

[11] S. Ervedoza, O. Glass, S. Guerrero, J.-P. Puel, Local exact controllability for the one-dimensional compressible Navier-Stokes equation, Arch. Ration. Mech. Anal. 206 (1) (2012) 189-238.

[12] E. Fernández-Cara, Motivation, analysis and control of the variable density Navier-Stokes equations, Discrete Contin. Dyn. Syst., Ser. S 5 (6) (2012) 1021-1090.

[13] E. Fernández-Cara, S. Guerrero, Global Carleman inequalities for parabolic systems and applications to controllability, SIAM J. Control Optim. 45 (4) (2006) 1399-1446 (electronic).

[14] E. Fernández-Cara, S. Guerrero, O.Y. Imanuvilov, J.-P. Puel, Local exact controllability of the Navier-Stokes system, J. Math. Pures Appl. (9) 83 (12) (2004) 1501-1542.

[15] E. Fernández-Cara, S. Guerrero, O.Y. Imanuvilov, J.-P. Puel, Some controllability results for the $N$-dimensional Navier-Stokes and Boussinesq systems with $N-1$ scalar controls, SIAM J. Control Optim. 45 (1) (2006) 146-173 (electronic).

[16] A.V. Fursikov, O.Yu. Èmanuilov, Exact controllability of the Navier-Stokes and Boussinesq equations, Usp. Mat. Nauk 54 (3(327)) (1999) 93-146.

[17] A.V. Fursikov, O.Y. Imanuvilov, Controllability of Evolution Equations, Lecture Notes Series, vol. 34, Seoul National University Research Institute of Mathematics Global Analysis Research Center, Seoul, 1996.

[18] A.V. Fursikov, O.Yu. Imanuvilov, Local exact boundary controllability of the Boussinesq equation, SIAM J. Control Optim. 36 (2) (1998) 391-421.

[19] V. Girault, P.-A. Raviart, Finite Element Methods for Navier-Stokes Equations. Theory and Algorithms, Springer, Berlin, 1986.

[20] O. Glass, Exact boundary controllability of 3-D Euler equation, ESAIM Control Optim. Calc. Var. 5 (2000) 1-44 (electronic).

[21] M. González-Burgos, S. Guerrero, J.-P. Puel, Local exact controllability to the trajectories of the Boussinesq system via a fictitious control on the divergence equation, Commun. Pure Appl. Anal. 8 (1) (2009) 311-333.

[22] O.Y. Imanuvilov, Remarks on exact controllability for the Navier-Stokes equations, ESAIM Control Optim. Calc. Var. 6 (2001) 39-72 (electronic).

[23] O.Y. Imanuvilov, J.-P. Puel, Global Carleman estimates for weak solutions of elliptic nonhomogeneous Dirichlet problems, Int. Math. Res. Not. 16 (2003) 883-913.

[24] O.Y. Imanuvilov, J.-P. Puel, M. Yamamoto, Carleman estimates for parabolic equations with nonhomogeneous boundary conditions, Chin. Ann. Math., Ser. B 30 (4) (2009) 333-378.

[25] O.Y. Imanuvilov, M. Yamamoto, Carleman inequalities for parabolic equations in Sobolev spaces of negative order and exact controllability for semilinear parabolic equations, Publ. Res. Inst. Math. Sci. 39 (2) (2003) 227-274.

[26] P. Martin, L. Rosier, P. Rouchon, Null controllability of the structurally damped wave equation with moving control, SIAM J. Control Optim. 51 (1) (2013) 660-684.

[27] M. Tucsnak, G. Weiss, Observation and Control for Operator Semigroups, Birkhäuser Advanced Texts, vol. XI, Springer, 2009.

[28] E. Zuazua, Log-Lipschitz regularity and uniqueness of the flow for a field in $\left(W_{\mathrm{loc}}^{n / p+1, p}\left(\mathbb{R}^{n}\right)\right)^{n}$, C. R. Math. Acad. Sci. Paris 335 (1) (2002) $17-22$. 